University of Louisville

ThinkIR: The University of Louisville's Institutional Repository

Electronic Theses and Dissertations

$12-2016$

\title{
Single carrier frequency domain equalization and energy efficiency optimization for MIMO cognitive radio.
}

Xiaohui Zhang

University of Louisville

Follow this and additional works at: https://ir.library.louisville.edu/etd

Part of the Power and Energy Commons, Signal Processing Commons, and the Systems and Communications Commons

\section{Recommended Citation}

Zhang, Xiaohui, "Single carrier frequency domain equalization and energy efficiency optimization for MIMO cognitive radio." (2016). Electronic Theses and Dissertations. Paper 2574.

https://doi.org/10.18297/etd/2574

This Doctoral Dissertation is brought to you for free and open access by ThinkIR: The University of Louisville's Institutional Repository. It has been accepted for inclusion in Electronic Theses and Dissertations by an authorized administrator of ThinkIR: The University of Louisville's Institutional Repository. This title appears here courtesy of the author, who has retained all other copyrights. For more information, please contact thinkir@louisville.edu. 


\title{
SINGLE CARRIER FREQUENCY DOMAIN EQUALIZATION AND ENERGY EFFICIENCY OPTIMIZATION FOR MIMO COGNITIVE RADIO
}

\author{
By \\ Xiaohui Zhang \\ A Dissertation \\ Submitted to the Faculty of the \\ J. B. Speed School of Engineering of the University of Louisville \\ in Partial Fulfillment of the Requirements \\ for the Degree of \\ Doctor of Philosophy in Electrical Engineering \\ Department of Electrical Engineering \\ University of Louisville \\ Louisville, Kentucky
}

December 2016 
Copyright 2016 by Xiaohui Zhang

All rights reserved 



\title{
SINGLE CARRIER FREQUENCY DOMAIN EQUALIZATION AND ENERGY EFFICIENCY OPTIMIZATION FOR MIMO COGNITIVE RADIO
}

\author{
By \\ Xiaohui Zhang \\ B.S., Zhengzhou University, 2008 \\ M.S., Zhengzhou University, 2012
}

A Dissertation Approved On

November 14, 2016

by the following Dissertation Committee:

Hongxiang Li, Dissertation Director

John Naber

Tamer Inanc

Lihui Bai 


\section{DEDICATION}

To all who love, support and help human beings to make the earth a better place for us. 


\section{ACKNOWLEDGMENTS}

I would like first to thank my advisor, Dr. Hongxiang Li, Assistant Professor, Department of Electrical and Computer Engineering at the University of Louisville, for his guidance, encouragement, and patience. I would also like to thank Dr. Johe Naber, Dr. Tamer Inanc, and Dr. Lihui Bai for agreeing to serve on the dissertation committee. I am grateful for their encouragement and advice. I am also grateful to my colleagues, Chen Cao and Wenqi Liu for their support during the research. At last, I would like to thank my parents for their great support. 


\section{ABSTRACT \\ SINGLE CARRIER FREQUENCY DOMAIN EQUALIZATION AND ENERGY \\ EFFICIENCY OPTIMIZATION FOR MIMO COGNITIVE RADIO \\ Xiaohui Zhang}

November 14, 2016

This dissertation studies two separate topics in wireless communication systems. One topic focuses on the Single Carrier Frequency Domain Equalization (SC-FDE), which is a promising technique to mitigate the multipath effect in the broadband wireless communication. Another topic targets on the energy efficiency optimization in a multiple input multiple output (MIMO) cognitive radio network.

For SC-FDE, the conventional linear receivers suffer from the noise amplification in deep fading channel. To overcome this, a fractional spaced frequency domain (FSFD) receiver based on frequency domain oversampling (FDO) is proposed for SC-FDE to improve the performance of the linear receiver under deep fading channels. By properly designing the guard interval, a larger sized Discrete Fourier Transform (DFT) is equipped to oversample the received signal in frequency domain. Thus, the effect of frequency-selective fading can still be eliminated by a one-tap frequency domain filter. Two types of FSFD receivers are proposed based on the least square (LS) and minimum mean square error (MMSE) criterion. Both the semi-analytical analysis and simulation results are given to evaluate the performance of the proposed receivers. Another challenge 
in SC-FDE is the in-phase/quadrature phase (IQ) imbalance caused by unideal radio frequency (RF) front-end at the transmitter or the receiver. Most existing works in single carrier transmission employ linear compensation methods, such as LS and MMSE, to combat the interference caused by IQ imbalance. Actually, for single carrier transmissions, it is possible for the receivers to adopt advanced nonlinear compensation methods to improve the system performance under IQ imbalance. For such purpose, an iterative decision feedback receiver is proposed to compensate the IQ imbalance caused by unideal RF front-end in SC-FDE. Numerical results show that the proposed iterative IQ imbalance compensation can significantly improve the performance of SC-FDE system under IQ imbalance compared with the conventional linear method.

For the energy efficiency optimization in the MIMO cognitive radio network, multiple secondary users (SUs) coexisting with a primary user (PU) adjust their antenna radiation patterns and power allocations to achieve energy-efficient transmission. The optimization problems are formulated to maximize the energy efficiency of a cognitive radio network in both distributed and centralized point of views. Also, constraints on the transmission power and the interference to $\mathrm{PU}$ are introduced to protect the PU's transmission. In order to solve the non-convex optimization problems, convex relaxations are used to transform them into equivalent problems with better tractability. Then three optimization algorithms are proposed to find the energy-efficient transmission strategies. Simulation results show that the proposed energy-efficiency optimization algorithms outperform the existing algorithms. 


\section{TABLE OF CONTENTS}

Page

ACKNOWLEDGMENTS

ABSTRACT V V

LIST OF FIGURES $\quad$ x

\section{CHAPTER}

I INTRODUCTION 1

A Multipath Channel ................. 2

B Single Carrier Frequency Domain Equalization . . . . . . . . . . 4

C Frequency Domain Oversampling ............. . . 4

D Spectrum Efficiency and Energy Efficiency . . . . . . . . . 6

E Cognitive Radio . . . . . . . . . . . . . . . 8

F Multiple Input Multiple Output . . . . . . . . . . . . . 9

G Outline .............................. 10

II FRACTIONALLY SPACED FREQUENCY DOMAIN RECEIVER FOR $\begin{array}{ll}\text { SC-FDE } & 12\end{array}$

A Background and Motivation ............... 12

B System Model . . . . . . . . . . . . . . . . . 15

C Equalization and Channel Estimation . . . . . . . . . . . 20

1 Equalization .................. 20

2 Semi-Analytical Performance Analysis . . . . . . . . . 21

3 Frequency domain channel estimation based on FDO . . . . . 24

D Simulation Results . . . . . . . . . . . . . 26

1 Static Channel ................ 26 
2 Rayleigh Fading Channel . . . . . . . . . . . . . . . . 28

3 Double Selective Channel . . . . . . . . . . . . . . . . . . . 29

4 Channel Estimation . . . . . . . . . . . . . . . . . 30

III ITERATIVE IQ IMBALANCE COMPENSATION RECEIVER FOR SCFDE

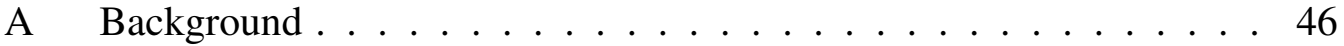

B System model . . . . . . . . . . . . . . . . . . . . . 49

$1 \quad$ Single Carrier Block Transmission With IQ Imbalance . . . 49

2 Frequency Domain Representation . . . . . . . . . . . . 54

C Iterative IQ Imbalance Compensation $\ldots \ldots \ldots \ldots$

1 Iterative Receiver Architecture . . . . . . . . . . . . 57

2 Hard Detection . . . . . . . . . . . . . . . . 61

3 Soft Detection . . . . . . . . . . . . . . . . 61

4 Computational Complexity . . . . . . . . . . . . . 62

D Joint IQ Imbalance and Channel Estimation . . . . . . . . . . . . 63

1 Frequency Domain Channel Estimation . . . . . . . . . 64

2 Time Domain Channel Estimation . . . . . . . . . . . . . 65

3 Impact Of IQ Imbalance On Channel Estimation $\ldots \ldots \ldots 6$

E Simulation ..................... 67

1 Perfect Channel Knowledge . . . . . . . . . . . 68

2 Channel Estimation . . . . . . . . . . . . . . . . 70

\section{ENERGY EFFICIENT TRANSMISSION STRATEGY FOR MIMO} COGNITIVE RADIO 81

A Background and Motivation $\ldots \ldots \ldots \ldots$. . . . . . 82

B System Model . . . . . . . . . . . . . . . . . . . . . . 84

1 Network Description . . . . . . . . . . . . . 84

2 Energy Efficiency Analysis . . . . . . . . . . . . . . 87

C Network Energy Efficiency Optimization _ . . . . . . . . . . 89 
D Sum of Energy Efficiency Optimization . . . . . . . . . . . 93

1 Problem Transformation ............... 93

2 Outer Loop Iteration . . . . . . . . . . . . . . 96

3 Solving the Subproblem . . . . . . . . . . . . . 98

E Distributed Energy Efficiency Optimization . . . . . . . . . . . . . 99

F Simulation Results . . . . . . . . . . . . . . . . . 101

V CONCLUSION AND FUTURE WORK 111

A Single Carrier Frequency Domain Equalization . . . . . . . . . 111

B Energy Efficient Transmission Strategy For MIMO Cognitive Radio . 114

REFERENCES

116

CURRICULUM VITAE

123 


\section{LIST OF FIGURES}

FIGURE $\quad$ Page

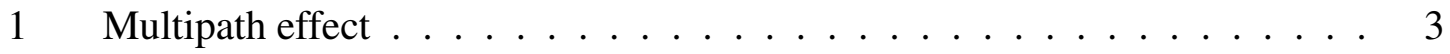

2 Block diagram of SC-FDE .................. 5

3 Frequency domain oversampling . . . . . . . . . . . . 7

4 Transmission beamforming ....................... 11

5 Block diagram of transceiver for proposed system. . . . . . . . . . . 32

6 Amplitude response of channel A . . . . . . . . . . . . . 33

7 BER of LS and MMSE receivers under channel A . . . . . . . . . . . . 34

8 Amplitude response of channel B . . . . . . . . . . . . . . . 35

9 BER of LS and MMSE receivers under channel B . . . . . . . . . . 36

10 BER of LS and MMSE receivers under channel C . . . . . . . . . . . . 37

11 Amplitude response of channel C . . . . . . . . . . . . . . 38

12 BER of LS and MMSE receivers with different oversampling rates under Rayleigh fading channel . . . . . . . . . . . . . . . 39

13 BER of LS and MMSE receivers versus oversampling rate under Rayleigh fading channel at three different SNRs . . . . . . . . . . . . . . 40

14 BER of LS and MMSE receivers under double selective channel . . . . . . 41

15 MSEs of time-domain channel estimation with different oversampling rates 42

16 MSEs of time-domain channel estimation versus oversampling rate R . . . 43

17 BER of MMSE receivers with different oversampling rates for channel estimation and equalization. . . . . . . . . . . . . . . 44

18 Block diagram of transceiver for single carrier transmission with IQ imbalance. . . . . . . . . . . . . . . 72

19 Block diagram of iterative IQ imbalance compensation receiver. . . . . . . 73 
20 IRR of the case 1 and case $2 \ldots \ldots \ldots \ldots \ldots$. . . . . . . . . . . . . . .

21 BERs of proposed receivers at the first four iterations with perfect CSI under case $1 \ldots \ldots \ldots \ldots$. . . . . . . . . . . . . . . . . . .

22 BER comparison of proposed receivers with conventional IBDFE receiver under case $1 \ldots \ldots \ldots \ldots \ldots$

23 BER comparison of proposed receivers with conventional IBDFE receiver under case $2 \ldots \ldots \ldots \ldots$. . . . . . . . . . . . . . . . . . . . . . . . .

24 BER comparison of proposed receivers with conventional IBDFE receiver under case $3 \ldots \ldots \ldots \ldots$. . . . . . . . . . . . . . . . . . . . . . . .

25 MSEs of frequency domain and time domain channel estimation . . . . . 79

26 BERs of proposed IQ imbalance compensation receivers with estimated CSI 80

27 Network Model. . . . . . . . . . . . . . . . . . . . . 85

28 (a)Throughput of one link versus network total transmitting power. (b) EE of one link versus network total transmitting power. . . . . . . . . . . 88

29 Convergence of the proposed Algorithms . . . . . . . . . . . . . 102

30 Sum of EE versus relative distance $A$. . . . . . . . . . . . . . . . 104

31 Network throughput versus relative distance. . . . . . . . . . . . 105

32 Sum of EE in Scenario 2 . . . . . . . . . . . . . . 106

33 Network throughput in Scenario 2. . . . . . . . . . . . . . . 107

34 Worst EE under different algorithms in Scenario 2. . . . . . . . . . . 108

35 Sum of EE under different algorithms in Scenario 3 . . . . . . . . . . . . . 109

36 Sum of EE under different algorithms in Scenario 4 . . . . . . . . . . . . 110 


\section{CHAPTER I}

\section{INTRODUCTION}

In the past few decades, wireless communications have been integrated into people's daily lives. Due to the widespread use of mobile devices, the demand for wireless service has exponentially increased and will continue to do to so. This will present the wireless communications with varied technical challenges from different aspects.

Firstly, new applications like video sharing, video streaming require high speed, low latency data transmission. High speed data transmission over wireless channels commonly faces the challenges of the multipath effect. Due to this, there is a need to design transmission techniques that can combat the adverse effects of the wireless channels. Conventional time domain equalization becomes unpractical due to its high complexity in high speed transmission. Orthogonal frequency division multiplexing (OFDM) has become widely accepted because of its robustness against frequency selective fading channels with low complexity, but it suffers a number of drawbacks such as high peak-to-average power ratio (PAPR). To overcome them, single carrier transmission with frequency domain equalization has been proposed as a promising alternative technique for OFDM.

In addition, the explosion of data transmission over wireless channels causes heavy burden on the limited spectrum resource. Conventional fixed spectrum assignment 
regulation results in a low spectrum efficiency. In order to improve the spectrum utilization efficiency, cognitive radio is proposed by allowing devices to dynamical access the spectrum. Furthermore, as the energy demand increases, the energy efficiency of communication systems becomes more and more important. The following will give an introduction to these challenges and the corresponding techniques to overcome them.

\section{A Multipath Channel}

Wireless channels generally have strong multipath effect, which is due to the presence of a wide range of natural and man-made objects. As shown in Figure 1, due to reflection, diffraction and scattering of surrounding objects, the signal sent from a base station arrives at the cellphone through different paths at different time. The result is the dispersion of the transmitted signal through the channel. When the symbol interval is smaller than the duration of dispersion, the multipath channel will cause inter-symbol interference (ISI) among transmitted data symbols, which makes it difficult to recover the data at the receiver. However, to achieve fast data transmission, small symbol intervals are inevitably used in most wireless systems. Therefore, the method used to recover the data corrupted by ISI is an important issue for broadband wireless communications.

Cyclic prefix (CP) assisted block transmission techniques employing frequency domain equalization (FDE) schemes are known to be excellent candidates for severe time-dispersive channels, allowing good performance and implementation complexity that is much lower than those of traditional time-domain equalization techniques. OFDM [1] is the most popular frequency-domain equalization technique. SC-FDE is another valuable candidate for highly dispersive channels in broadband wireless communications [2] [3]. In 


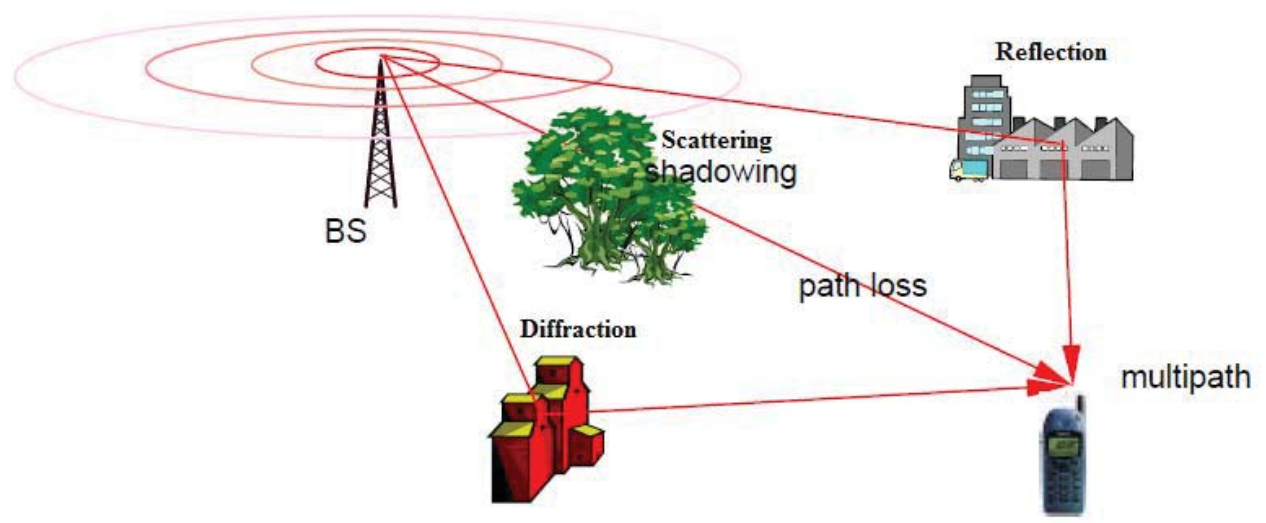

Figure 1: Multipath effect

both cases, a CP is appended to each block, eliminating the inter-block interference and converting the linear convolution that is associated with the channel into a circular convolution with respect to the useful part of the transmitted block. This allows low complexity Fast Fourier Transform (FFT) based receiver implementations.

\section{B Single Carrier Frequency Domain Equalization}

Similar to OFDM, SC-FDE also performs the channel equalization in the frequency domain to mitigate the effect of multipath with low complexity, but it is different from OFDM that, in SC-FDE, both the FFT and inverse FFT (IFFT) locate at the receiver. Its transceiver scheme is shown in Figure 2. At the transmitter, it sends the information data serially in the time domain. Then, at the receiver, it transforms the received data into the frequency domain to eliminate the effect of multipath channel. So it has low computational complexity and avoids the drawback of high peak to average power rate (PAPR) and being sensitive to the frequency offset [4]. As such, SC-FDE enables the use of a cheaper power amplifier than a comparable OFDM system; and this is a benefit of some importance, since the power amplifier can be one of the most costly components in a consumer broadband 


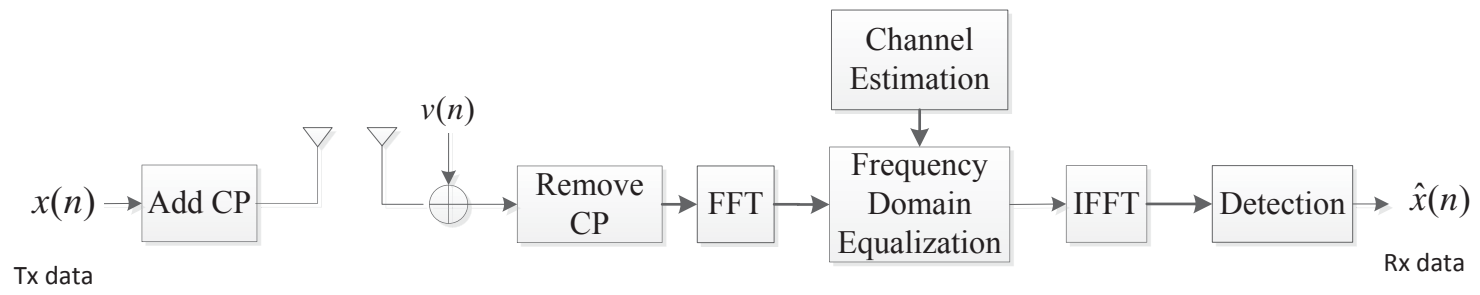

Figure 2: Block diagram of SC-FDE

wireless transceiver [3]. Due to these attracting features, SC-FDE has become one of the important channel equalization techniques in the physical layer of the broadband wireless communication system.

C Frequency Domain Oversampling

It is well known that the Discrete Fourier Transform (DFT) is the frequency domain samples of a sequence, while the sampling number equals to the length of DFT. For example, Figure 3 illustrates the basic idea of frequency domain oversampling (FDO). A time domain sequence with length of $N$ is shown in Figure 3(a). Its corresponding frequency domain representation is continuous as shown in Figure 3(b). Let $R$ be the oversampling rate. Without overampling, i.e. $R=1$, taking a $N$-point DFT to the time domain sequence results in $N$ frequency domain samples as shown in Figure 3(c). When $R>1$, a zero-padded DFT is used to taken more than $N$ samples in the frequency domain. Figure 3(d) shows the $2 N$ frequency domain samples with $R=2$. It can be observed that FDO actually introduces redundancy in the frequency domain, which may increase the robustness of signal processing.

Recently, FDO was introduced into OFDM to improve the detection performance [5-10]. It has been shown that frequency diversity inherent in a multipath channel can be 


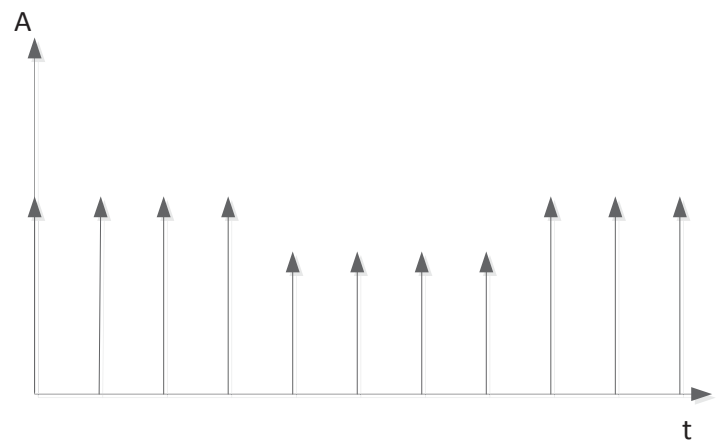

(a). Time domain signal

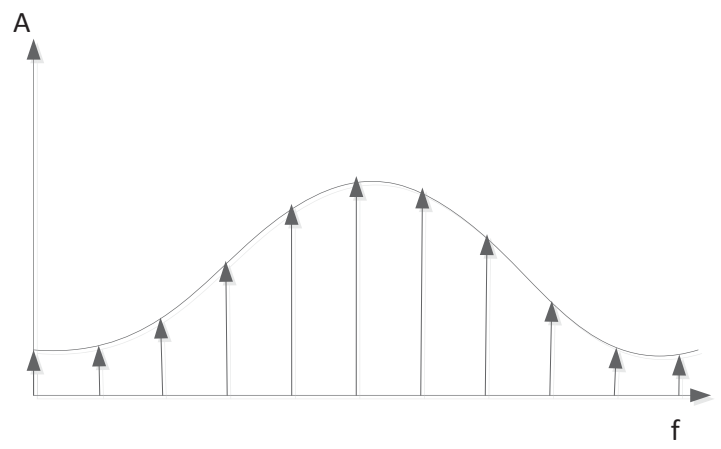

(c). Frequency domain samples with $\mathrm{R}=1$

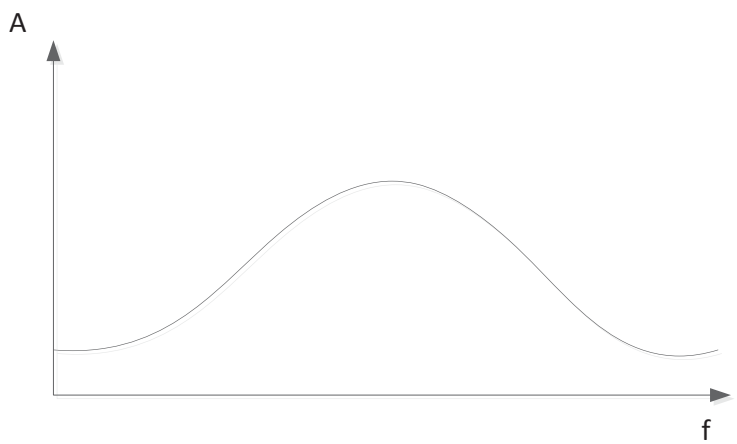

(b). Frequency domain signal

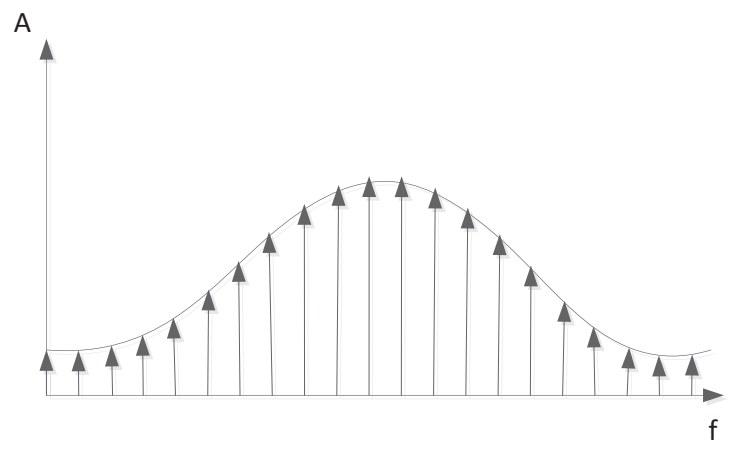

(d). Frequency domain samples with $\mathrm{R}=2$

Figure 3: Frequency domain oversampling

extracted and exploited by using frequency domain oversampling. This diversity advantage outweighs the effect of inter-carrier interference generated by frequency-domain oversampling, due largely to the minimum mean squared error (MMSE) receivers' interference suppression capability. Numerical results show that the FDO based MMSE receiver outperforms the conventional MMSE receiver in OFDM under both ideal and practical situations. However, in SC-FDE, the effect of FDO has rarely been studied.

\section{Spectrum Efficiency and Energy Efficiency}

Due to physical-natural constraints, there are portions of the spectrum that simply cannot be used for wireless communications. As as result, the spectrum band suitable for 
electromagnetic signal transmission is limited. There are quantitative evidences showing that the wireless data explosion is real and will continue. According to annual visual network index (VNI) report released by Cisco (Feb. 2014), in just a decade, the amount of IP data handled by wireless networks will increase by over a factor of 100: from under 3 exabytes in 2010 to over 190 exabytes by 2018, on pace to exceed 500 exabytes by 2020 and forecast that the incremental approaches will not come close to meet the demands that networks will face by 2020 [11]. Therefore, it is urgent for engineers to design new techniques and improve the spectrum efficiency of wireless communication systems, so that the limited frequency bands can be further explored to fulfill the increasing demand of wireless services.

On the other hand, green communication has become one of the main design considerations of the communication systems in the future. Particularly, because of the widespread use of mobile devices, efficient wireless transceivers with low energy consumption have become critical to prolong the battery life. With the explosive growth of data traffic accompanied and the rapidly increasing of energy consumption, energy efficiency has become increasingly important in future communication systems. The energy efficiency of a communication network can be measured in terms of bit-per-joule capacity, which is the maximum amount of bit that can be delivered by per joule energy consumed by the communication network [12].

\section{E Cognitive Radio}

The current spectrum regulation uses a fixed spectrum assignment policy, in which different wireless networks are assigned licenses to operate on different frequency bands 
so that they will not interfere with each other. However, this fix spectrum usage is quite inefficient. As a fact, some of licensed frequency bands are seldom used, while other frequency bands are heavily occupied [13].

In order to overcome this drawback, cognitive radio has been proposed as a promising technique that can largely improve the efficiency of spectrum utilization. In cognitive radio, the spectrum access can be configured dynamically and it allows the unlicensed users to share the spectrum of licensed users. The licensed users are usually called primary users, which means it has the priority to use a specific frequency band. While the unlicensed users are called secondary users, it implied that the unlicensed users have permission to access the licensed frequency band only if the licensed users' transmission requirement is met. Cognitive radio usually can work under two modes: overlay mode and underlay mode. In overlay mode, a secondary user can only access the frequency band when it is not used by the primary user. For underlay mode, a secondary user can access the frequency band under the condition that the secondary user can control its interference to primary user under certain level, so that the primarily user's transmission would not be disturbed.

\section{F Multiple Input Multiple Output}

Multiple Input Multiple Output (MIMO) is an important method to improve performance of wireless communication systems. In a MIMO system, multiple antennas equipped on both transmitter and receiver can largely improve the spectrum efficiency compared with conventional single antenna systems. A $N \times N$ MIMO channel can be seen as $N$ independent single antenna channels, which can be used for either increasing 
the diversity to combat channel fading, or employing spatial multiplexing to increase the data rate.

For diversity, multiple antennas send signal that carry the same information through different signal path from the transmitter to the receiver. Then multiple independently faded replicas of the data symbol can be obtained at the receiver. Therefore, more reliable reception is achieved [14]. For multiplexing, a transmitter sends multiple data streams through different antennas to the receiver. Multiple parallel spatial channels can be created, since the path gain between each individual transmit-receiver antenna pair fade independently. By using multiplexing of MIMO channel, the system throughput can largely be improved.

Another important feature of MIMO is that both transmitter and receiver can employ beamforming for interference suppression. By using beamforming, the transmitter can send signal towards the direction of its intended receiver and avoid interference to others by controlling the signal radiation pattern as shown in Figure 4 . The receiver can also extract signal of interest from the desired direction and suppress the interference from other transmission. In a multi-user setting, this allow the multiple users coexisting on the same frequency band by exploit the spacial diversity and avoid to interfere with each other.

\section{G Outline}

The rest of this dissertation is organized as follows. Chapter II investigates the frequency domain oversampling in SC-FDE. Two linear receivers based on frequency domain oversamping are proposed according to the least square (LS) and minimum mean square error (MMSE) criterion. Theoretical analysis and simulation results on 


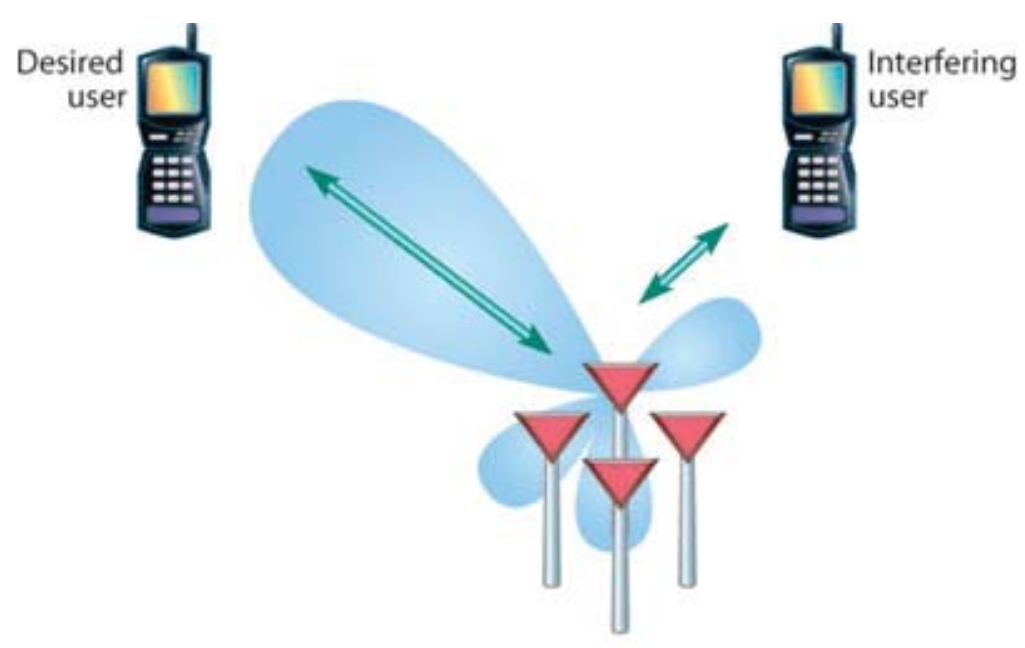

Figure 4: Transmission beamforming

bit-error-rate are given to evaluate the performance of proposed receivers. Chapter III discusses the compensation of in-phase and quadrature (IQ) imbalance caused by unideal RF front-end in SC-FDE. It first gives the system model of SC-FDE with frequency dependent IQ imbalance at both the transmitter and receiver; derives an iterative compensation method to jointly mitigate the impact of IQ imbalance and multipath channel using a decision feedback scheme; then provides numerical results for performance evaluation. In Chapter IV, the energy efficiency optimization in MIMO cognitive radio is discussed. In order to optimize the energy efficiency under interference channels, it proposes three different algorithms based on different optimization objectives and provides simulation results to show the effectiveness of the proposed algorithms. Finally, the conclusion and thoughts on future works are given in Chapter V. 


\section{CHAPTER II}

\section{FRACTIONALLY SPACED FREQUENCY DOMAIN RECEIVER FOR \\ SC-FDE}

SC-FDE is an alternative technique to orthogonal frequency division multiplex (OFDM) for broadband wireless channels to mitigate the intersymbol interference (ISI) [2]. It has similar performance and signal processing complexity as OFDM, but avoids OFDM drawbacks such as high peak-to-average power ratio and sensitivity to carrier frequency offset [15][16]. Due to these desired properties, SC-FDE has been adopted by 3GPP LTE for uplink transmissions [17]. Within the framework of SC-FDE, this chapter applies FDO to SC-FDE to improve the equalization performance of SC-FDE under multipath channels.

\section{A Background and Motivation}

FDO is a common conception in signal processing and can be easily applied through zero-padded Discrete Fourier Transform (DFT) [18]. However, FDO had not obtained much attention in wireless communication systems until it was introduced to OFDM systems [5-10]. Specifically, Lottici V. et al. uses FDO to estimate the frequency offset of OFDM [5]. By exploiting the structure of multiple access interference (MAI), Shi Q. et al. [6] designs a FDO based receiver to suppress MAI for the uplink MC-CDMA 
system. In [7], the authors explore FDO to design the linear minimum mean square error single-user detection for downlink MC-CDMA system, where three types of detectors with different performance/complexity tradeoff are proposed. Furthermore, it shows that a FDO based MMSE receiver for OFDM can better exploit the frequency diversity and outperform the conventional OFDM receivers under various channel scenarios [8]. Recently, FDO was adopted to improve the OFDM performance over underwater acoustic channel with large Doppler spread in [9] and two way relay channel in [10]. All these results have demonstrated that FDO is a promising technique to improve the performance of OFDM systems. However, as pointed out in [8], FDO also largely increases the computational complexity of OFDM system. Since the channel matrix cannot be diagonalized anymore and the frequency domain data lose their orthogonality.

Compared with its multicarrier counterpart, FDO has rarely been studied in singlecarrier communication systems. In fact, some practical communication systems prefer single-carrier transmissions due to various system considerations and tradeoffs. In [19], FDO is applied to SC-FDE for the first time and the preliminary results have shown that FDO can improve the performance of channel estimation and equalization over frequencyselective channels. Built upon [19], this chapter will thoroughly study the effect of FDO in SC-FDE. Specifically, two types of FDO based fractional spaced frequency domain (FSFD) receivers will be designed using MMSE and least square (LS) criteria. Meanwhile, it will provide performance analysis for the proposed FSFD receivers and study the effect of FDO from the frequency diversity perspective. Note that SC-FDE itself can exploit frequency diversity because frequency-domain equalization and time-domain detection are operated separately [20]. However, SC-FDE systems usually cannot take the full frequency diversity 


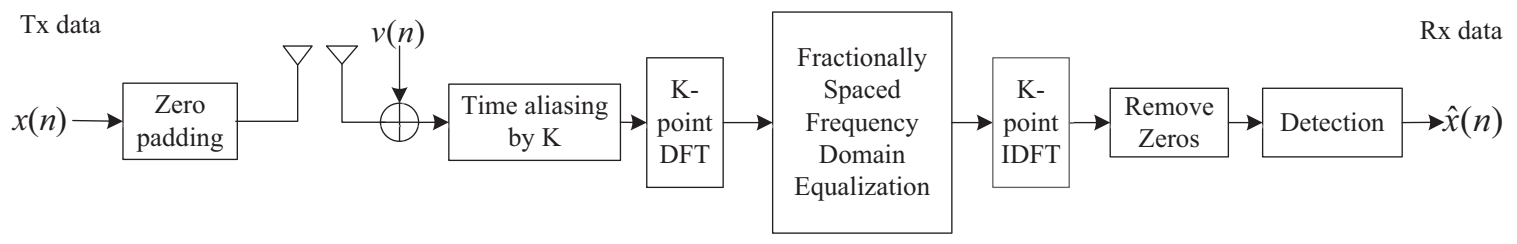

Figure 5: Block diagram of transceiver for proposed system.

gain, which is a function of transmission rate and data block length (i.e. DFT size) [21]. In Particular, given transmission rate, the diversity gain of SC-FDE can be improved by increasing the DFT size.

In the proposed FSFD receiver for SC-FDE, zero-padding (ZP) instead of cyclic prefix $(\mathrm{CP})$ is chosen as guard interval so that the received signal can be represented as linear convolution of the channel impulse response and the transmitted signal. To keep the low complexity of frequency domain equalization, it aliases the received data to transform the linear convolution into circular convolution. Then a larger sized DFT is employed to oversample the received signal in frequency domain. Due to the time aliasing introduced at receiver, the oversampled frequency domain data can still be equalized by a one-tap filter via linear LS or MMSE criteria. Therefore, the added complexity due to oversampling is trivial compared with that in OFDM. Simulation results show that the proposed receivers can significantly improve the system's robustness against multipath channel with spectrum nulls. And both frequency domain oversampling based LS and MMSE receivers outperform the conventional SC-FDE in various channel environment.

To facilitate the discussion, it uses the following notation convention: $\{\cdot\}^{T},\{\cdot\}^{*}$, $\{\cdot\}^{H}$ respectively denote transpose, conjugate and conjugate transpose of a matrix; $*, \otimes$ and $\mathbb{E}[\cdot]$ respectively represent convolution, circular convolution and statistical expectation; 
$\mathbf{I}_{N \times N}$ and $\mathbf{0}_{N \times M}$ respectively stand for identity matrix (with size of $N \times N$ ) and all-zero matrix (with size of $N \times M$ ); $\operatorname{diag}(\cdot)$ returns a square diagonal matrix with the elements of vector on the main diagonal.

\section{B System Model}

For a single-input and single-output system, Figure 5 depicts the signal block diagram of the proposed SC-FDE transceiver. At transmitter, each data block of length $N$ is padded with a number of zeros as guard interval to avoid inter-block interference (IBI) before being transmitted over frequency-selective fading channels. At the receiver, all received data symbols, including those corresponding to guard interval, are processed. Specifically, the received data are first pre-processed by time aliasing with period of $K$ $(K \geq N)$. Then, a $K$-point DFT is applied to convert the time domain signal into frequency domain. Due to time aliasing and the larger sized DFT, the signal is oversampled in frequency domain with oversampling rate $R=K / N$. Then the FDO based frequency-domain equalization is employed to mitigate ISI, followed by an inverse DFT (IDFT) to convert the equalized frequency-domain signal into time-domain. Finally, those symbols corresponding to guard interval are discarded and the rest are used for detection and estimation. The output is the estimated data sequence.

Let $x(n)$ be the $n^{t h}$ transmitted symbol at the current block whose average power is $\sigma_{s}^{2}$, and $y(n)$ be the $n^{\text {th }}$ received baseband signal at the current block. Since ZP is chosen as guard interval, it has

$$
x(n)=0, N+1 \leq n \leq N+N_{z},
$$

where $N_{z}$ is the length of guard interval. 
Assume that the frequency-selective channel is constant during one data block, and its baseband equivalent channel impulse response is $h(n)$ with length $L$, where $\sum_{l=1}^{L} h(l)^{2}=1$. To avoid IBI, the minimum length of the guard interval is $N_{z}=L-1$. Accordingly, the received baseband signal is given by

$$
y(n)=h(n) * x(n)+v(n)
$$

where $v(n)$ is the additive white Gaussian noise (AWGN) with average power of $\sigma_{n}^{2}$. Let $M=N+N_{z}$, equation (2) can be expressed in matrix form as

$$
\mathbf{y}=\mathbf{H x}+\mathbf{v}
$$

where

$$
\begin{aligned}
& \mathbf{y}=[y(1) y(2) y(3) \ldots y(M)]^{T}, \\
& \mathbf{x}=[x(1) x(2) x(3) \ldots x(N)]^{T}, \\
& \mathbf{v}=[v(1) v(2) v(3) \ldots v(M)]^{T},
\end{aligned}
$$

and $\mathbf{H}$ is an $M \times N$ Toeplitz matrix with first column $[h(1), h(2), \ldots, h(L), 0, \ldots, 0]^{T}$.

In equation (2), ignoring the noise component, the received signal is the linear convolution of the transmitted signal and the channel impulse response. As is well known, the circular convolution of two finite-length sequences is equivalent to linear convolution of the two sequences, followed by time aliasing [22]. Therefore, at the receiver side, the $K$-point circular convolution of $h(n)$ and $x(n)$ can be obtained by time aliasing:

$$
\bar{y}(n)=h(n) \otimes x(n)+\bar{v}(n)
$$

When $N \leq K<M$, time aliasing represents an overlap-add operation, which is implemented by cutting off the last $(M-K)$ points and adding them to the first $(M-K)$ 
points of the current data block. Thus, $\bar{y}(n)$ and $\bar{v}(n)$ in equation (7) are given as:

$$
\begin{aligned}
& \bar{y}(n)= \begin{cases}y(n)+y(K+n), & 1 \leq n \leq M-K \\
y(n), & M-K<n \leq K\end{cases} \\
& \bar{v}(n)=\left\{\begin{array}{ll}
v(n)+v(K+n), & 1 \leq n \leq M-K \\
v(n), & M-K<n \leq K
\end{array} .\right.
\end{aligned}
$$

When $K \geq M$, the received signal is padded with $(K-M)$ zeros. Accordingly, $\bar{y}(n)$ and $\bar{v}(n)$ in equation (7) become:

$$
\begin{aligned}
& \bar{y}(n)= \begin{cases}y(n), & 1 \leq n \leq M \\
0, & M<n \leq K\end{cases} \\
& \bar{v}(n)= \begin{cases}v(n), & 1 \leq n \leq M \\
0, & M<n \leq K\end{cases}
\end{aligned}
$$

The matrix form of equation (7) can be expressed as

$$
\overline{\mathbf{y}}=\mathbf{T} \overline{\mathbf{x}}+\overline{\mathbf{v}}
$$

where $\mathbf{T}$ is a $K \times K$ circulated time-domain channel gain matrix, given by

$$
\mathbf{T}=\left(\begin{array}{ccccccc}
h(1) & 0 & \cdots & 0 & h(L) & \cdots & h(2) \\
h(2) & h(1) & 0 & \ddots & \ddots & \ddots & \vdots \\
\vdots & \ddots & \ddots & \ddots & \ddots & \ddots & h(L) \\
h(L) & \ddots & \ddots & h(1) & 0 & \ddots & 0 \\
0 & h(L) & \ddots & \ddots & \ddots & \ddots & \vdots \\
\vdots & \ddots & \ddots & \ddots & \ddots & \ddots & 0 \\
0 & \cdots & 0 & h(L) & \cdots & \cdots & h(1)
\end{array}\right)
$$


and

$$
\begin{aligned}
& \overline{\mathbf{y}}=\boldsymbol{\Xi}_{2} \mathbf{y} \\
& \overline{\mathbf{x}}=\boldsymbol{\Xi}_{1} \mathbf{x} \\
& \overline{\mathbf{v}}=\boldsymbol{\Xi}_{2} \mathbf{v}
\end{aligned}
$$

with $\Xi_{1}$ representing “zero-padding” operation:

$$
\boldsymbol{\Xi}_{1}=\left(\begin{array}{ll}
\mathbf{I}_{N \times N} & \mathbf{0}_{N \times(K-N)}
\end{array}\right)^{T}
$$

and $\Xi_{2}$ representing either "overlap-add" or "zero-padding" operation (depending on the value of $K)$ :

- $N \leq K<M$

$$
\boldsymbol{\Xi}_{2}=\left(\begin{array}{ll} 
& \mathbf{I}_{(M-K) \times(M-K)} \\
\mathbf{I}_{K \times K} & \\
& \mathbf{0}_{(2 K-M) \times(M-K)}
\end{array}\right)
$$

- $K \geq M$

$$
\boldsymbol{\Xi}_{2}=\left(\begin{array}{ll}
\mathbf{I}_{M \times M} & \mathbf{0}_{M \times(K-M)}
\end{array}\right)^{T}
$$

Remark 1. When $K=N$, the proposed FSFD system becomes the conventional ZP-SCFDE without frequency-domain oversampling [23].

Define the normalized $K \times K$ DFT matrix as

$$
\begin{array}{r}
\mathbf{F} \triangleq\left\{\frac{1}{\sqrt{K}} \exp \left(-j \frac{2 \pi}{K} k n\right)\right\}_{K \times K}, \\
k, n=0,1, \cdots, K-1
\end{array}
$$


Taking the DFT of the received signal and noticing that $\mathbf{F}^{H} \mathbf{F}=\mathbf{I}$, the frequency-domain representation of equation (12) can be obtained as

$$
\mathbf{Y}=\mathbf{F} \overline{\mathbf{y}}=\mathbf{F T F}^{H} \mathbf{F} \overline{\mathbf{x}}+\mathbf{F} \overline{\mathbf{v}}=\mathbf{H X}+\mathbf{V}
$$

where $\mathbf{H}=\mathbf{F T F}^{H}$ is the frequency-domain channel matrix with oversampling rate $R=$ $K / N$, and $\mathbf{V}=\mathbf{F} \overline{\mathbf{v}}$ denotes the zero-mean white Gaussian noise vector with covariance matrix

$$
\mathbf{C}=\sigma_{n}^{2} \mathbf{F} \boldsymbol{\Xi}_{2} \boldsymbol{\Xi}_{2}^{H} \mathbf{F}^{H}
$$

For $N \leq K<M, \mathbf{V}$ is still zero-mean white Gaussian noise; for $K \geq M$, due to the zero-padding in the time domain, $\mathbf{V}$ becomes a colored zero-mean Gaussian noise vector. Note that the average power of frequency-domain data $X_{K}(k)$ and $V_{K}(k)$ is reduced to $\frac{N \sigma_{s}^{2}}{K}$ and $\frac{M \sigma_{n}^{2}}{K}$, respectively.

Since $\mathbf{T}$ is circulant, $\mathbf{H}$ is diagonal. The frequency-domain input-output relationship can be simplified as

$$
Y_{K}(k)=H_{K}(k) X_{K}(k)+V_{K}(k), k=1,2, \cdots, K,
$$

where

$$
\begin{aligned}
& Y_{K}(k)=\frac{1}{\sqrt{K}} \sum_{n=1}^{K} \bar{y}(n) \exp \left(\frac{-j 2 \pi(n-1)(k-1)}{K}\right) \\
& X_{K}(k)=\frac{1}{\sqrt{K}} \sum_{n=1}^{N} x(n) \exp \left(\frac{-j 2 \pi(n-1)(k-1)}{K}\right) \\
& V_{K}(k)=\frac{1}{\sqrt{K}} \sum_{n=1}^{K} \bar{v}(n) \exp \left(\frac{-j 2 \pi(n-1)(k-1)}{K}\right)
\end{aligned}
$$

and $H_{K}(k)$ is the DFT of the channel response

$$
H_{K}(k)=\sum_{l=1}^{L} h(l) \exp \left(\frac{-j 2 \pi(l-1)(k-1)}{K}\right) .
$$


The expression of frequency domain signal $Y_{K}(k)$ in equation (23) suggests that $X_{K}(k)$ can be estimated by a one-tap filter. The estimation of $X_{K}(k)$ is given as

$$
\hat{X}_{K}(k)=\omega(k) Y_{K}(k), k=1,2, \cdots, K,
$$

where $\omega(k)$ is the $k^{t h}$ weight of the one-tap filter to be determined.

Finally the IDFT is taken to transform $\hat{X}_{k}(k)$ into time-domain, and the estimation of the zero-padded time-domain data is given as

$$
\hat{\overline{\mathbf{x}}}=\mathbf{F}^{H} \hat{\mathbf{X}}_{K}
$$

where

$$
\hat{\mathbf{X}}_{K}=\left[\hat{X}_{K}(1) \hat{X}_{K}(2) \cdots \hat{X}_{K}(N) \cdots \hat{X}_{K}(K)\right]^{T} .
$$

Note that the first $N$ symbols of $\hat{\overline{\mathbf{x}}}$ correspond to the information data and the rest $(K-N)$ symbols are discarded. Therefore, the estimation of $\mathbf{x}$ is given as

$$
\hat{\mathbf{x}}=\mathbf{F}_{K \times N}^{H} \hat{\mathbf{X}}_{K}
$$

where $\mathbf{F}_{K \times N}$ is a $K \times N$ matrix consisting of the first $N$ columns of $\mathbf{F}$.

\section{Equalization and Channel Estimation}

This section designs the LS and MMSE based FSFD equalization for the proposed SC-FDE system, conduct a semi-analytical performance analysis and discuss the FDO based channel estimation.

\section{Equalization}

In principle, if the receiver has perfect knowledge of the channel state information (CSI), the frequency domain equalization filter $\omega(k)$ can be determined via linear LS (also 
known as zero forcing (ZF)) or MMSE criteria. Specifically, the LS equalization filter is given as

$$
\omega(k)=\frac{1}{H_{K}(k)}, \quad k=1,2, \cdots, K .
$$

Despite its simplicity, it is well known that LS equalization suffers from noise enhancement [23]. That is, when the multipath channel experiences deep fading, the noise power will be significantly increased. Alternatively, the MMSE equalizer provides better tradeoff between ISI mitigation and noise enhancement. The MMSE equalization filter is given as

$$
\omega(k)=\frac{H_{K}^{*}(k)}{\left|H_{K}(k)\right|^{2}+\frac{\sigma_{n}^{2}}{\sigma_{s}^{2}}}, \quad k=1,2, \cdots, K .
$$

Remark 2. Compared with the conventional cyclic prefix based SC-FDE (CP-SC-FDE), the computational complexity of the proposed transceiver is higher due to a larger DFT size used for FDO. Specifically, the complexity for CP-SC-FDE and the FDO based SC-FDE are respectively $\mathcal{O}\left(N \log _{2}(N)\right)$ and $\mathcal{O}\left(K \log _{2}(K)\right)$ so that the added complexity depends on the oversampling rate $R$. However, as shown in equation (23), the frequency-domain channel matrix can still be diagonalized so that the conventional channel estimation and equalization methods are still applicable in the proposed SC-FDE system based on FDO.

2 Semi-Analytical Performance Analysis

In this section, the performance of the proposed receiver in terms of its bit error rate (BER) is analyzed.

Substituting equation (15) and (16) into (21) obtains

$$
\begin{aligned}
\mathbf{Y} & =\mathbf{H F} \boldsymbol{\Xi}_{1} \mathbf{x}+\mathbf{F} \boldsymbol{\Xi}_{2} \mathbf{v} \\
& =\mathbf{H F}_{K \times N} \mathbf{x}+\mathbf{F} \boldsymbol{\Xi}_{2} \mathbf{v}
\end{aligned}
$$


Let $\mathbf{W}=\operatorname{diag}\{\omega(1) \omega(2) \cdots \omega(K)\}$ be the equalization weight matrix, the frequency domain input signal is estimated as

$$
\begin{aligned}
\hat{\mathbf{X}}_{K} & =\mathbf{W Y} \\
& =\mathbf{W H F}_{K \times N} \mathbf{x}+\mathbf{W F} \boldsymbol{\Xi}_{2} \mathbf{v} .
\end{aligned}
$$

Plugging equation (35) into (31) obtains

$$
\begin{aligned}
\hat{\mathbf{x}} & =\mathbf{F}_{K \times N}^{H} \mathbf{W} \mathbf{H} \mathbf{F}_{K \times N} \mathbf{x}+\mathbf{F}_{K \times N}^{H} \mathbf{W F} \mathbf{\Xi}_{2} \mathbf{v} \\
& =\mathbf{A} \mathbf{x}+\boldsymbol{\eta},
\end{aligned}
$$

with

$$
\begin{gathered}
\mathbf{A}=\mathbf{F}_{K \times N}^{H} \mathbf{W H F}_{K \times N} \\
\boldsymbol{\eta}=\mathbf{F}_{K \times N}^{H} \mathbf{W F} \boldsymbol{\Xi}_{2} \mathbf{v},
\end{gathered}
$$

where $\boldsymbol{\eta}$ has the following covariance matrix

$$
\begin{aligned}
\mathbf{C}_{\eta} & =\mathbb{E}\left[\boldsymbol{\eta} \boldsymbol{\eta}^{H}\right] \\
& =\sigma_{n}^{2} \mathbf{F}_{K \times N}^{H} \mathbf{W F} \boldsymbol{\Xi}_{2} \boldsymbol{\Xi}_{2}^{H} \mathbf{F}^{H} \mathbf{W}^{H} \mathbf{F}_{K \times N} .
\end{aligned}
$$

Notice that the $N \times N$ matrix $\mathbf{A}$ in equation (37) is a function of the equalization weight matrix $\mathbf{W}$ and the channel gain matrix $\mathbf{H}$. Given the equalization criterion (e.g. LS or MMSE) and oversampling rate, matrix $\mathbf{A}$ will be solely determined by $\mathbf{H}$ if the receiver knows the channel state information. In practice, $\mathbf{A}$ is usually not diagonal due to the imperfect CSI or frequency offset, which introduces interference from adjacent symbols. In either case, the semi-analytical performance analysis can be given by approximating the interference as a Gaussian random variable [8]. Specifically, by using the Lyapunov Theorem [24], the interference for the $n^{t h}$ symbol can be approximated by a conditional 
zero mean Gaussian variable with the following variance:

$$
\begin{aligned}
\left.\sigma_{I, n}^{2}\right|_{\mathbf{H}} & =\mathbb{E}\left[\left\{\sum_{n^{\prime}=1, n^{\prime} \neq n}^{N} A\left(n, n^{\prime}\right) x\left(n^{\prime}\right)\right\}^{2}\right] \\
& =\sigma_{s}^{2} \sum_{n^{\prime}=1, n^{\prime} \neq n}^{N}\left|A\left(n, n^{\prime}\right)\right|^{2}
\end{aligned}
$$

Then the conditional signal to interference plus noise ratio (SINR) for the $n^{\text {th }}$ symbol is given as

$$
\left.\gamma_{n}\right|_{\mathbf{H}}=\frac{|A(n, n)|^{2}}{\sum_{n^{\prime}=1, n^{\prime} \neq n}^{N}\left|A\left(n, n^{\prime}\right)\right|^{2}+\mathbf{C}_{\eta}(n, n) / \sigma_{s}^{2}}
$$

According to equations (37) and (39), it can be seen from equation (41) that $\left.\gamma_{n}\right|_{\mathbf{H}}$ is determined by $\mathbf{W}$ and $\boldsymbol{\Xi}_{2}$. Given equalization criterion and oversampling rate, $\boldsymbol{\Xi}_{2}$ can be obtained by equation (18) for $1 \leq R<\frac{M}{N}$ and by (16) for $R \geq \frac{M}{N}$; W can be obtained by equation (32) for LS equalization and by equation (33) for MMSE equalization.

To get further insight on the BER performance, take an example where LS equalization is adopted and the oversampling rate satisfies $R \geq \frac{M}{N}$ (i.e. $K \geq M$ ). In this case, equalization weight matrix $\mathbf{W}=\mathbf{H}^{-1}$ and $\Xi_{2}$ becomes

$$
\Xi_{2}=\left(\begin{array}{ll}
\mathbf{I}_{M \times M} & \mathbf{0}_{M \times K-M}
\end{array}\right)^{T} .
$$

Then, it follows that

$$
\mathbf{A}=\mathbf{I}_{N \times N}
$$

Substituting equation (42) and (43) into equation (36) gives

$$
\hat{\mathbf{x}}_{L S}=\mathbf{x}+\mathbf{F}_{K \times N}^{H} \mathbf{W F}_{K \times M} \mathbf{v}
$$

where $\mathbf{F}_{K \times M}$ consists of the first $M$ columns of DFT matrix $\mathbf{F}$. The noise term in equation (44) is $\eta^{\prime}=\mathbf{F}_{K \times N}^{H} \mathbf{W F}_{K \times M} \mathbf{v}$ and its covariance matrix is given by

$$
\mathbf{C}_{\eta}=\sigma_{n}^{2} \mathbf{F}_{K \times N}^{H} \mathbf{W F}_{K \times M} \mathbf{F}_{K \times M}^{H} \mathbf{W}^{H} \mathbf{F}_{K \times N}
$$


Since $\mathbf{A}$ is a diagonal matrix, there is no ISI on each symbol. Thus, the SINR for the $n^{\text {th }}$ symbol is given as

$$
\left.\gamma_{n}\right|_{\mathbf{H}}=\frac{\sigma_{s}^{2}}{\mathbf{C}_{\eta}(n, n)} .
$$

Similarly, the SINR for other cases (i.e. MMSE or LS, $1 \leq R<\frac{M}{N}$ or $R \geq \frac{M}{N}$ ) can also be obtained.

According to the BER expressions in [25], the conditional BER of the $n^{\text {th }}$ symbol for MPSK and QAM constellations can respectively be derived as:

$$
\begin{gathered}
\left.P_{n}\right|_{\mathbf{H}} \simeq \frac{2}{\max \left(\log _{2} M, 2\right)} \times \sum_{i=1}^{\max (M / 4,1)} Q\left(\sqrt{\left.2 \gamma_{n}\right|_{\mathbf{H}}} \sin \frac{(2 i-1) \pi}{M}\right) \quad(M P S K) \\
\left.P_{n}\right|_{\mathbf{H}} \simeq 4\left(\frac{\sqrt{M}-1}{\sqrt{M}}\right)\left(\frac{1}{\log _{2} M}\right) \times \sum_{i=1}^{\sqrt{M} / 2} Q\left((2 i-1) \sqrt{\frac{\left.3 \gamma_{n}\right|_{\mathbf{H}}}{M-1}}\right) \quad(Q A M)
\end{gathered}
$$

where $Q(x)=(1 / 2 \pi) \int_{x}^{\infty} \exp \left(-\left(u^{2} / 2\right)\right) d u$. Note that the conditioning on channel matrix H can be removed by Monte Carlo integration.

3 Frequency domain channel estimation based on FDO

According to equation (23), the pilot-assisted channel estimation via MMSE criterion is given as

$$
\hat{H}_{K}(k)=\frac{X_{K}^{*}(k) Y_{K}(k)}{\left|X_{K}(k)\right|^{2}+\frac{\sigma_{n}^{2}}{\sigma_{s}^{2}}}
$$

The estimator $\hat{H}_{K}(k)$ can be improved by a frequency-domain filter to reduce the noise. Specifically, a common technique is to convert $\hat{H}_{K}(k)$ into time domain by IDFT and use an L-size window mask to remove the noise beyond the channel length, then the time-domain channel coefficients are converted back to frequency domain by DFT. This approach was originally proposed in OFDM systems in [26]. According to this method, in 
the FDO based SC-FDE, the time-domain channel response is estimated as

$$
\hat{\mathbf{h}}=\frac{1}{\sqrt{K}} \mathbf{F}_{K \times L}^{H} \boldsymbol{\xi}
$$

where $\boldsymbol{\xi}=\left[\hat{H}_{K}(1) \hat{H}_{K}(2) \cdots \hat{H}_{K}(K)\right]$ and $\mathbf{F}_{K \times L}$ is a $K \times L$ matrix consisting of the first $L$ columns of $\mathbf{F}$.

Thus, the improved frequency-domain channel estimation is

$$
\xi_{I}=\sqrt{K} \mathbf{F} \boldsymbol{\mu}
$$

where $\boldsymbol{\mu}=\left[\hat{\mathbf{h}}^{T} \mathbf{0}_{1 \times(K-L)}\right]^{T}$.

Remark 3. It has been proven that this method can reduce the average noise power of the estimator by a factor $(K / L)$ [15]. Therefore, compared with the conventional SC-FDE system $(K=N)$, the proposed SC-FDE system with FDO $(K>N)$ yields a larger reduction of noise power. Meanwhile, the proposed system provides more flexibility because the frequency-domain oversampling rate of channel equalization is not necessarily the same as that of channel estimation. In other words, different oversampling rate can be adopted at the training block (for channel estimation) and the data block (for channel equalization).

Due to the different DFT length used for FDO, it is inconvenient to evaluate the performance of channel estimation in frequency-domain. As a result, Euqation (50) is used to compare the performance of FDO based channel estimation with different oversampling rate in time-domain. The mean square error (MSE) of time-domain channel estimation $\hat{\mathbf{h}}$ is given as

$$
M S E=\mathbb{E}\left[(\hat{\mathbf{h}}-\mathbf{h})^{H}(\hat{\mathbf{h}}-\mathbf{h})\right]
$$




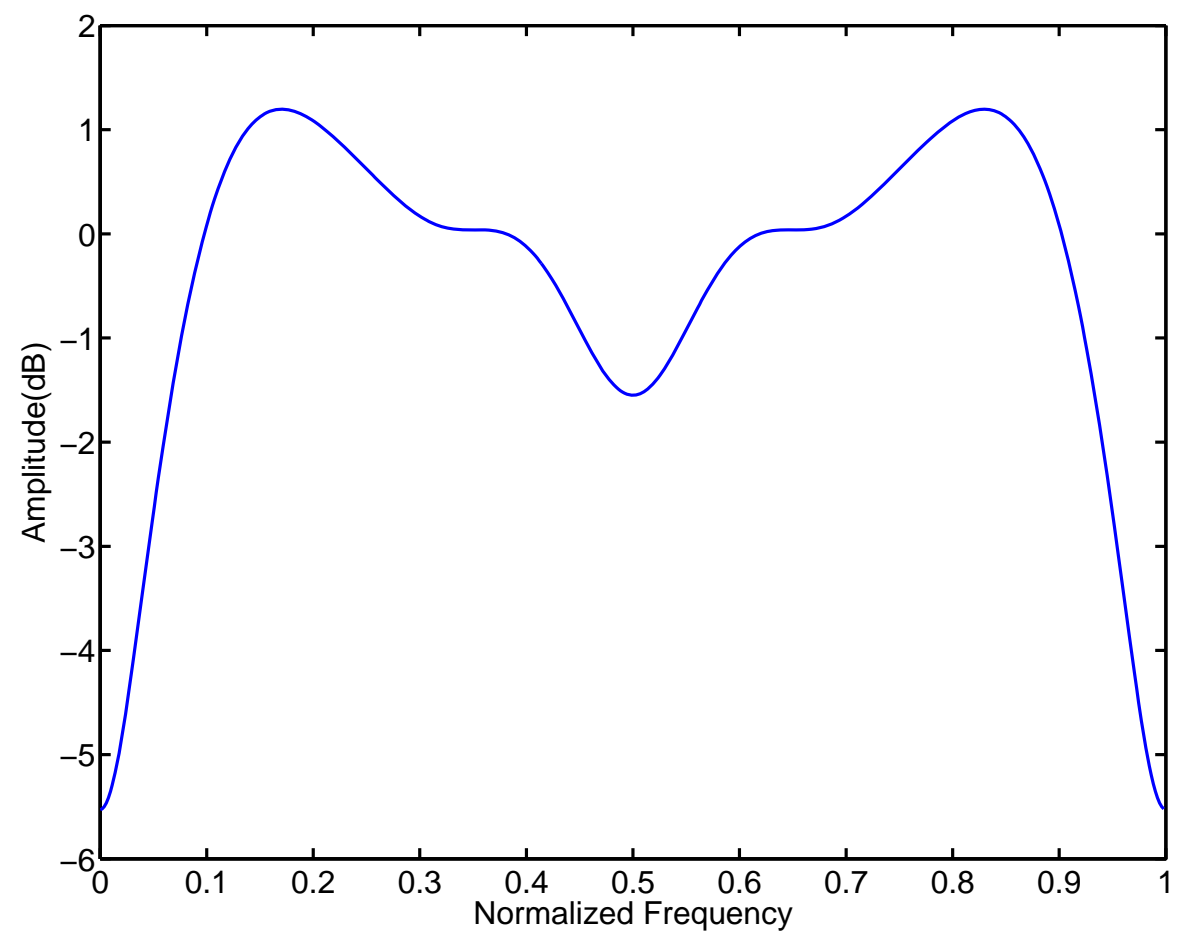

Figure 6: Amplitude response of channel A

D Simulation Results

In this section, the performance of the proposed SC-FDE receiver with oversampling rate $R>1$ is evaluated to compare with conventional SC-FDE without oversampling (i.e. $\quad R=1$ ). Specifically, consider uncoded QPSK modulation for single-carrier block transmissions with block size $N=64$. The length of ZP guard interval is set to be the channel maximum delay, which is $L-1$. Unless otherwise stated, assume perfect CSI at receiver side. When the oversampling rate $R=1$, the conventional CP-SC-FDE is adopted; when $R \geq 1$, the proposed ZP-SC-FDE is used. Extensive Monte Carlo simulation and semi-analytical results are provided at different oversampling rates for static channel, Rayleigh fading channel, double-selective channel and imperfect channel knowledge. 


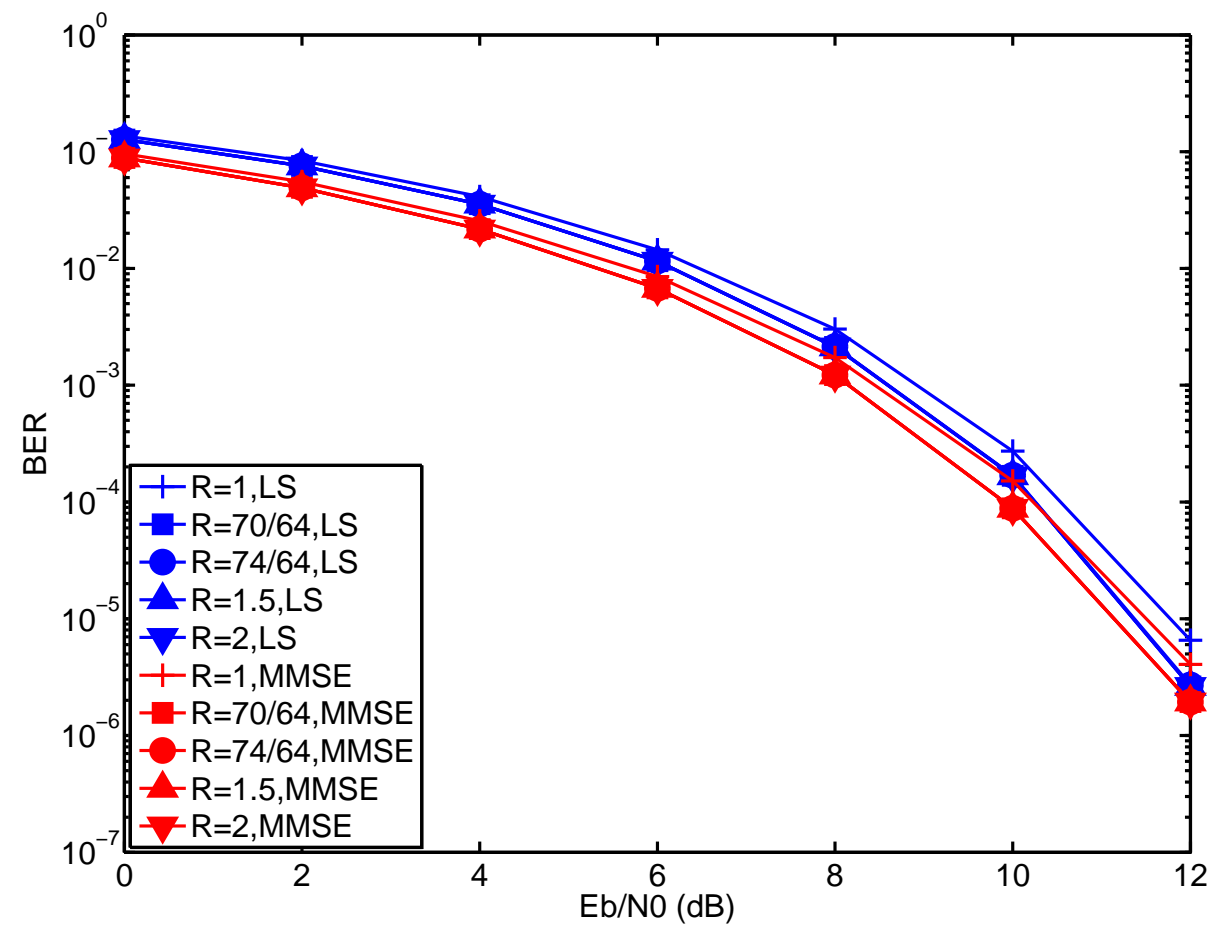

Figure 7: BER of LS and MMSE receivers under channel A

\section{Static Channel}

Consider three static multipath channel models (channel A, B and C). For channel A, its discrete impulse response is given by $(0.04,-0.05,0.07,-0.21,-0.5,0.72,0.36,0.0$, $0.21,0.03,0.03)[27]$. The corresponding frequency-domain amplitude response is shown in Figure 6, which has a mild amplitude distortion. The BER performance of the proposed receiver with different oversampling rate is given in Figure 7. It can be observed that, both LS and MMSE FSFD receivers with $R>1$ outperform the conventional CP-SC-FDE receiver with $R=1$. Furthermore, LS receiver with FDO can even outperform the MMSE receiver of CP-SC-FDE at high SNR region. Notice that in Channel A, both the LS and MMSE receivers achieve the maximum performance at oversampling rate $R=70 / 64$.

Unlike channel A, channel B is a 16 order sparse multipath channel with higher 


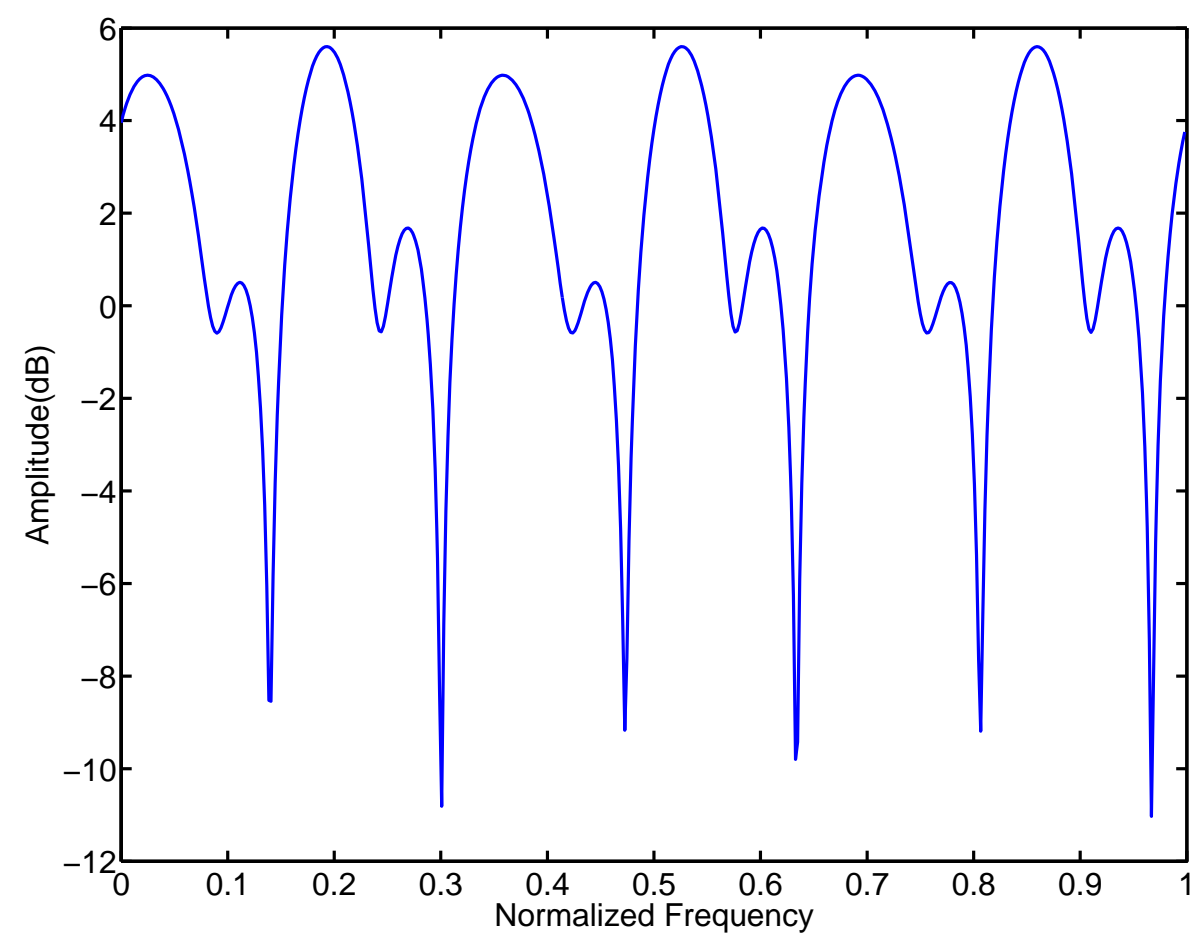

Figure 8: Amplitude response of channel B

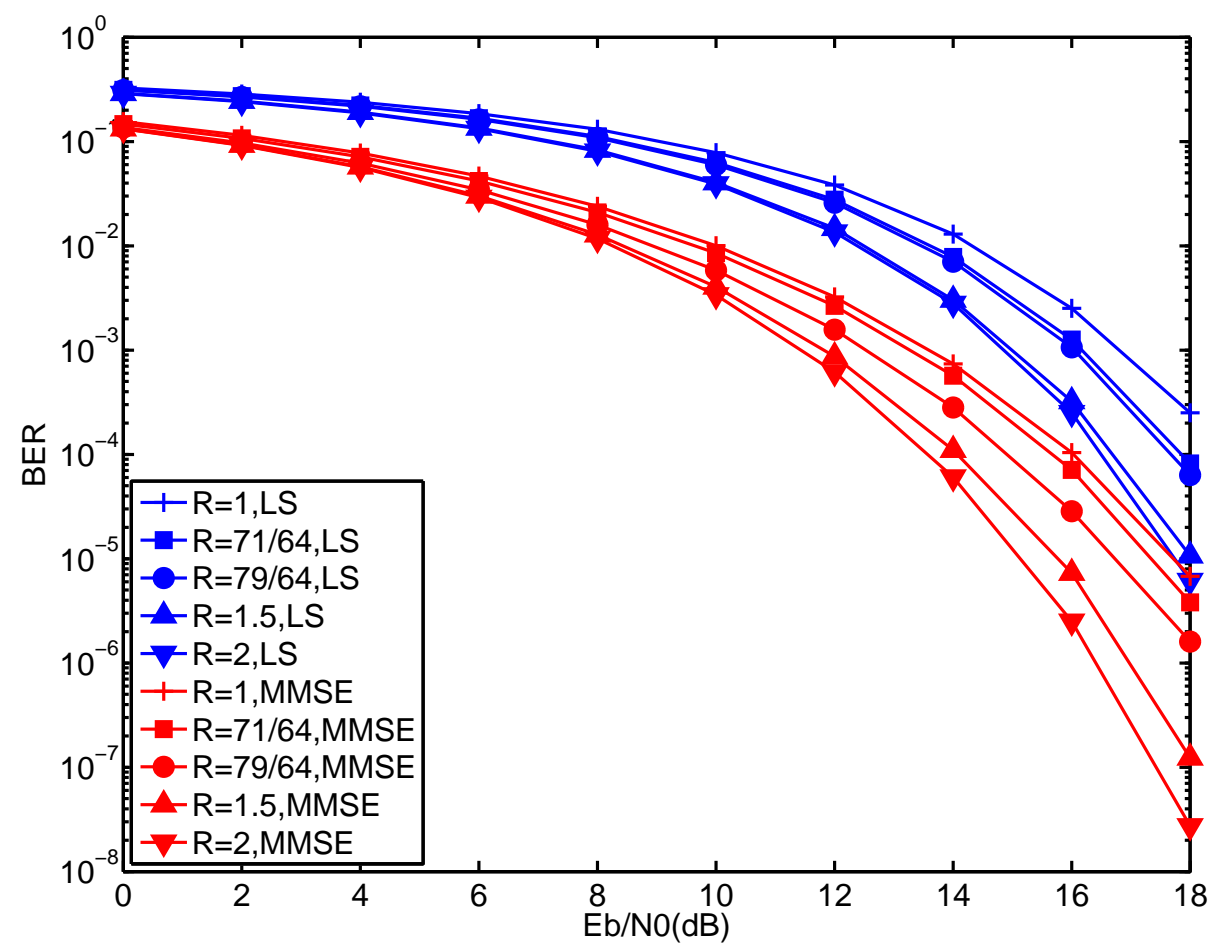

Figure 9: BER of LS and MMSE receivers under channel B 
distortion in frequency domain, as shown in Figure 8. Its discrete impulse response is ($0.65+1.26 \mathrm{i}, 0,0,0,0,0,-1.15-0.27 \mathrm{i}, 0,0,0,0,0,-0.59-0.59 \mathrm{i}, 0,0,-0.0026-0.24 \mathrm{i})$. The BER performance comparisons with different oversampling rates are shown in Figure 9. It can be seen that, for both LS and MMSE receivers, the BER performance increases with the oversampling rate increasing from 1 to 2 . Particularly, the LS receiver with $R=2$ achieves the same performance as the conventional MMSE receiver at $E_{b} / N_{0}=18 \mathrm{~dB}$.

Finally, Channel $\mathrm{C}$ with all paths having equal strength is adopted, which represents the worst-case of frequency-selective channel [28]. The normalized baseband representation of its impulse response is given as

$$
h(l)=\frac{1+j}{\sqrt{2 L}}, \quad 1 \leq l \leq L
$$

where the channel length is $L=16$.

In this case, the BER curves of LS and MMSE receiver with different oversampling rates are illustrated in Figure 10. For the LS equalization, it shows that the system cannot work at all (i.e. the BER curve remains constant at 0.5 ) when $R=1$. When increase the oversampling rate to $75 / 64$ and $79 / 64$, the performance is significantly improved. However, the BER performance goes back to 0.5 for $R=1.5$ and $R=2$. Therefore, for channel $\mathrm{C}$, a higher oversampling rate may not yield better performance. To get some insights, Figure 11(a) plots the frequency response of channel C, which is a Sinc function with a number of spectrum nulls. It is well known that the $K$-point DFT of the channel impulse response is the $K$-point equal spaced sampling of the channel frequency response. When $K=16$, Figure 11 shows that all the frequency nulls are sampled, which is also the case for $K=64,96$ or 128 (i.e. $R=1,1.5$ and 2). In these cases, the noise on frequency nulls are infinitely amplified after frequency domain equalization so that the 


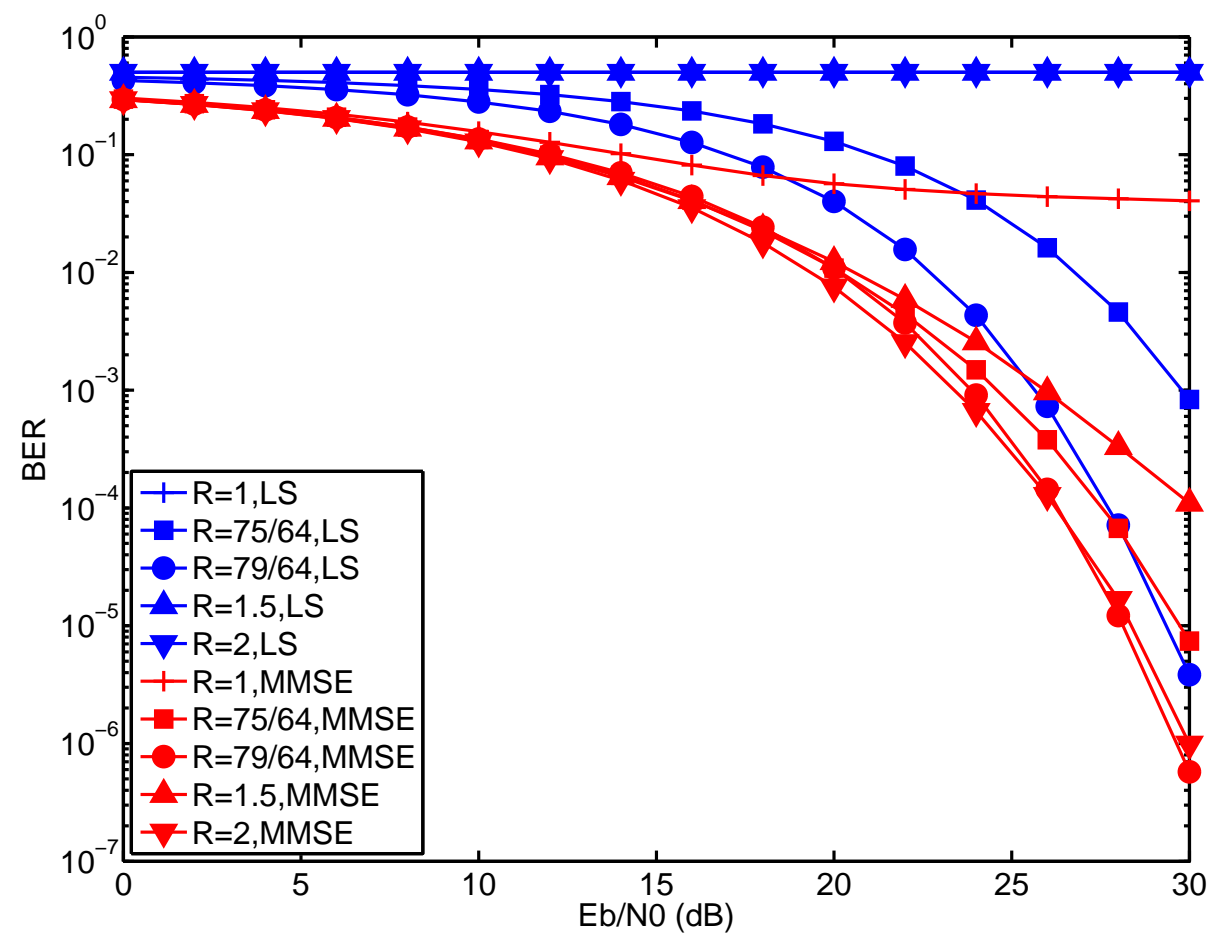

Figure 10: BER of LS and MMSE receivers under channel C

desired signal can not be recovered. On the other hand, when $K=19$, Figure 11(c) shows that most of the frequency nulls are avoided, which is also the case for $K=75$ or $K=79$ when the proposed LS receiver outperforms the conventional CP-SC-FDE receiver. For the MMSE receiver, the performance of system with FDO is also much better than that of the conventional CP-SC-FDE receiver. However, the performance of MMSE receiver with $R=2$ is not the best due to the same reason.

Remark 4. With FDO, the energy of the received signal is distributed over more frequency domain samples. In other words, a larger oversampling rate results in a more scattered energy distribution in frequency domain, which improves the frequency diversity gain. Among the three static channel models, the BER performance gain from FDO is more distinguished in channel $B$ and $C$ than that in channel A. This is because the 

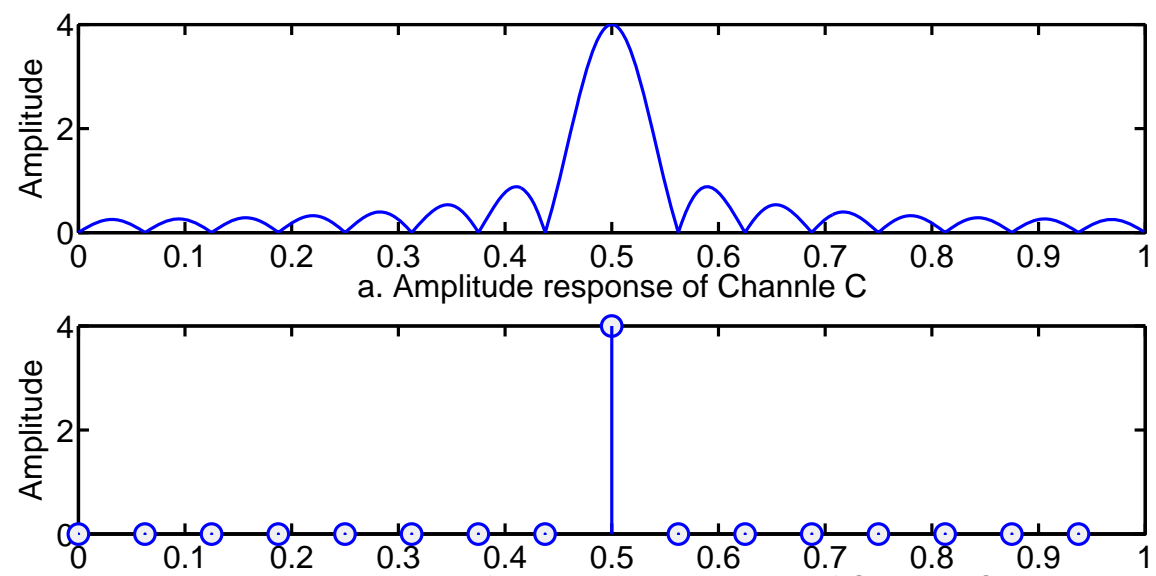

b. 16-point DFT of the impulse response of Channel $\mathrm{C}$

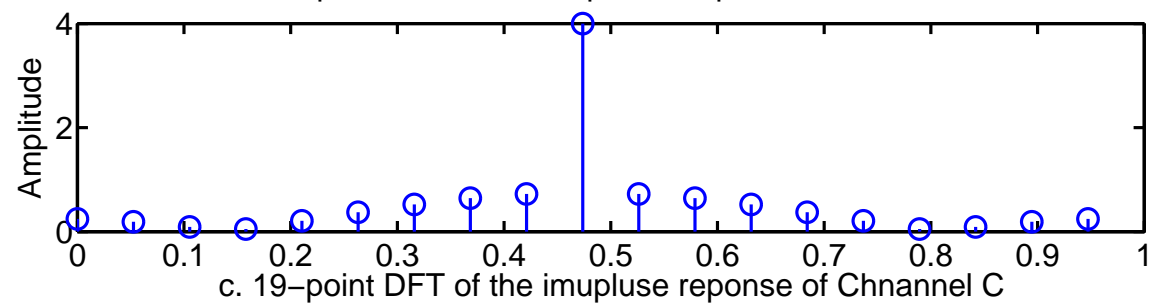

Figure 11: Amplitude response of channel C

inherent frequency diversity gain in channel $B$ and $C$ is higher than that in channel $A$ (i.e., channel $B$ and $C$ have more spectrum nulls and stronger amplitude distortion in frequency domain). Furthermore, as shown in the results of channel C, FDO rate can be properly adjusted to avoid sampling at spectrum nulls and reduce noise amplification during frequency-domain equalization.

\section{Rayleigh Fading Channel}

For quasi-static channel, consider a Rayleigh fading channel with uniform power delay profile (i.e., $\mathbb{E}\left[|h(l)|^{2}\right]=\sigma_{L}^{2}, \forall l \in\{1,2, \cdots, L\}$ ) and length $L=16$ [8]. To evaluate the performance of the proposed FSFD receiver, the BER curves are averaged over $10^{5}$ channel realizations. Figure 12 shows the BER performance of LS and MMSE 
receivers versus $E_{b} / N_{0}$ with oversampling rates $R=1,71 / 64,79 / 64,2$ and 5 . Semi-analytical results based on equation (48) are also plotted, which perfectly match the simulation.

From Figure 12, the following observations can be given: (1) FDO improves the BER performance for both LS and MMSE receivers. (2) For LS receiver, the performance gradually improves as $R$ increases from 1 to 5. (3) For MMSE receiver, the BER of FSFD receiver $(R \geq 1)$ also outperforms the conventional CP-SC-FDE receiver $(R=1)$. However, there is no performance improvement when the oversampling rate is increased from 2 to 5 .

In order to further study the effect of oversampling rate on LS and MMSE receivers, Figure 13 gives the BER versus oversampling rate varying from 1 to 10 at $E_{b} / N_{0}=10 \mathrm{~dB}, 15 \mathrm{~dB}$ and $20 \mathrm{~dB}$. It can been seen that, for LS equalization, the BER degrades as oversampling rate increases for all different SNR value and the performance gain with $R=10$ is almost $5 \mathrm{~dB}$, compared with that of $R=1$. For MMSE equalization, the FSFD receiver achieve its maximum performance at $R=2$.

\section{Double Selective Channel}

When mobility is involved in wireless communications, it introduces Doppler spread so that the channel is time varying within each data block. With multipath and Doppler spread, the channel becomes both frequency selective and time selective (i.e. double selective channel). This section uses Jake's model to generate the double selective channel and simulates the performance of the proposed FSFD receiver for SC-FDE. Specifically, adopt the 11-tap Rayleigh fading channel model described in [29], whose $l^{\text {th }}$ 


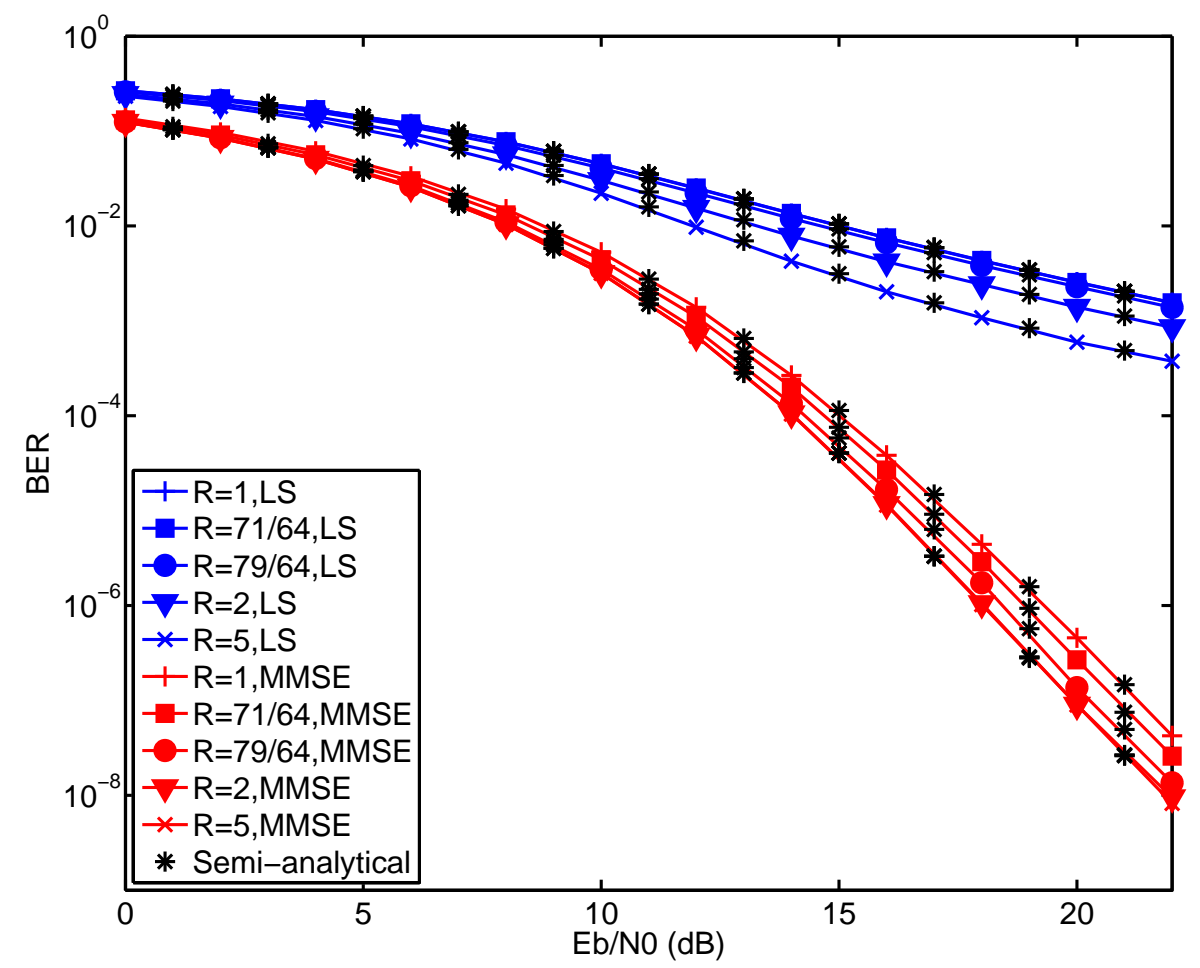

Figure 12: BER of LS and MMSE receivers with different oversampling rates under Rayleigh fading channel

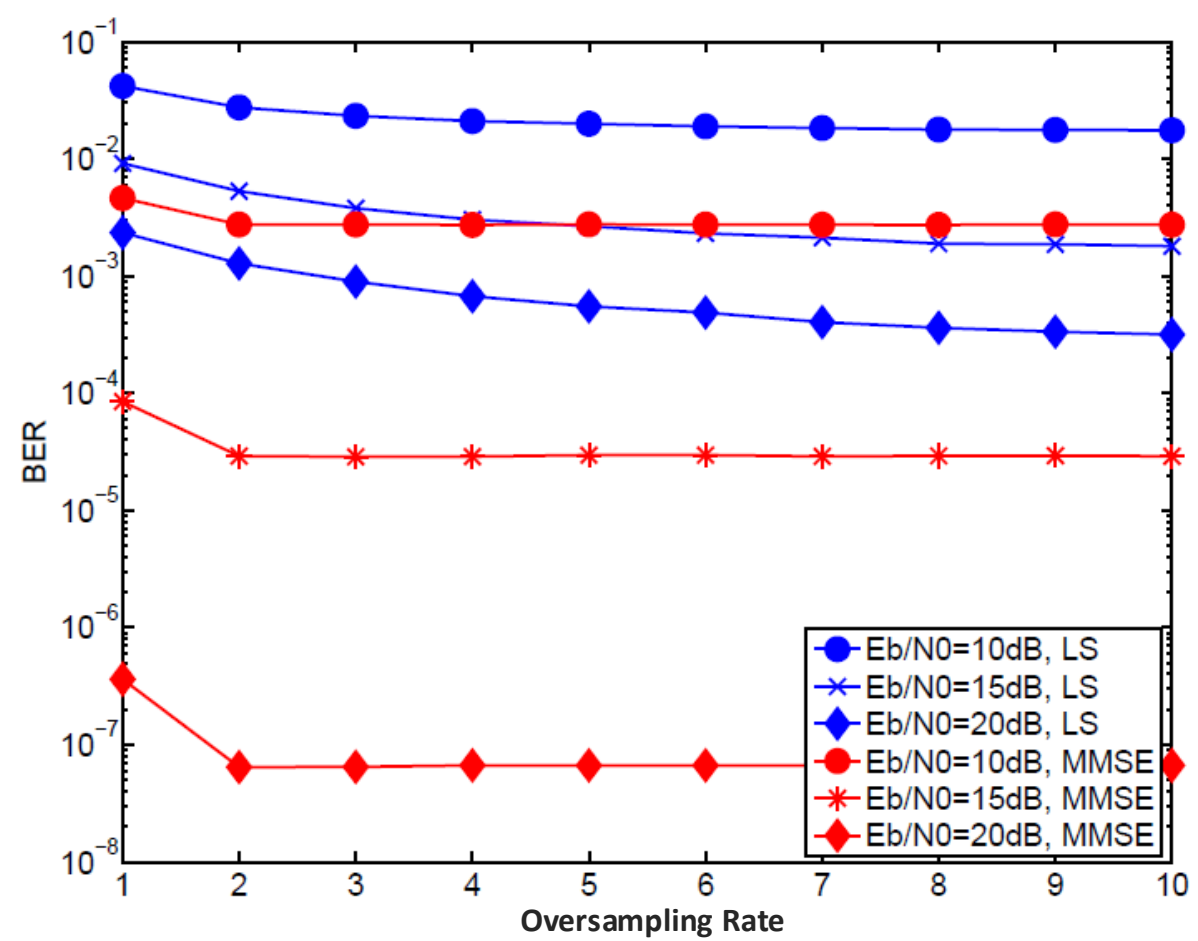

Figure 13: BER of LS and MMSE receivers versus oversampling rate under Rayleigh fading channel at three different SNRs 


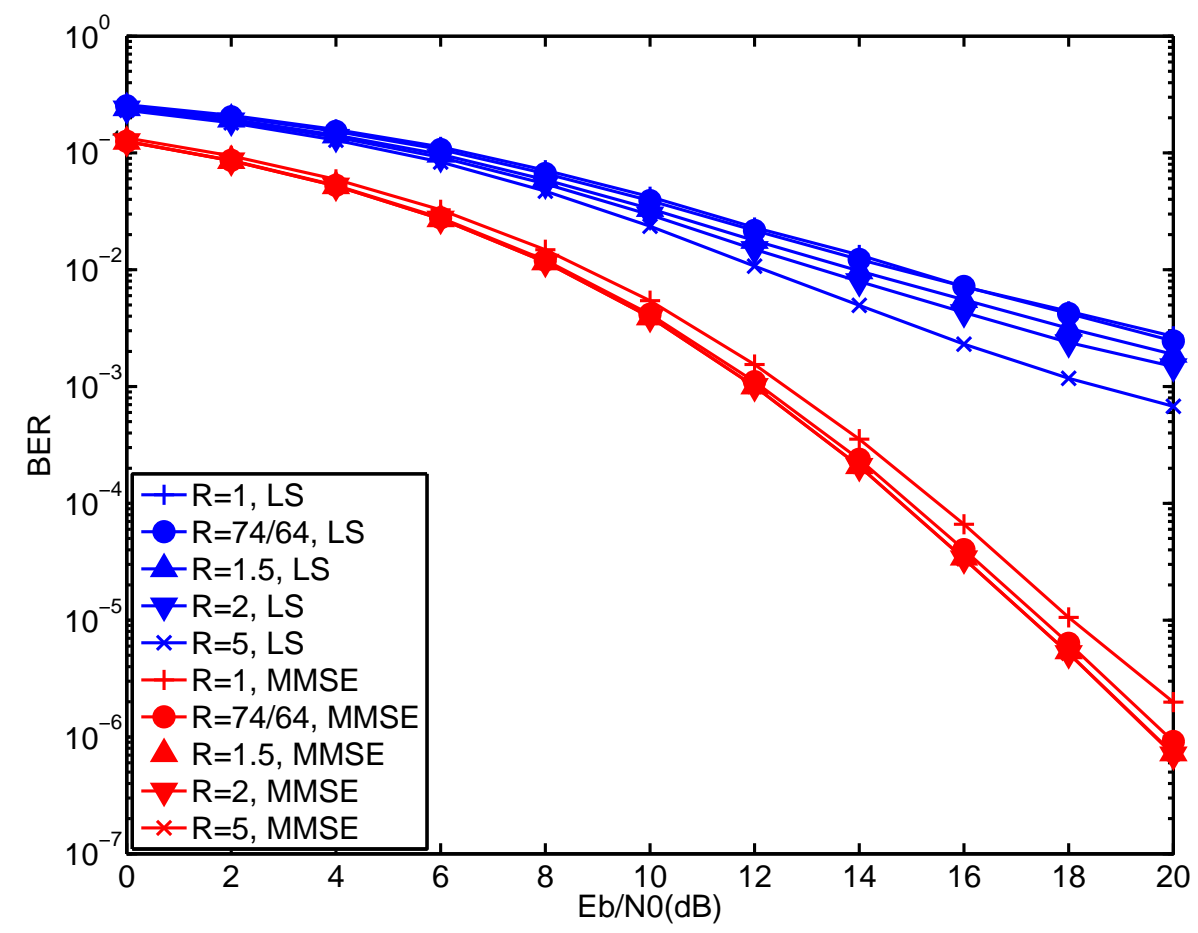

Figure 14: BER of LS and MMSE receivers under double selective channel

tap has an average power of $1.2257 \exp (-0.8 l)$ with the normalized maximum Doppler spread $f_{d} T_{s}=5 \times 10^{-5}$. Figure 14 shows that the performance of both LS and MMSE receivers can be improved by FDO over double-selective channel. Similar to the results in quasi-static channel, MMSE receiver can achieved its maximum performance at a moderate oversampling rate $(R=1.5)$, while LS receiver can still get performance gain by further increasing the oversampling rate from 2 to 5 .

\section{Channel Estimation}

This section evaluates the proposed channel estimation based on FDO, where the training sequence is randomly generated QPSK signals. Figure 15 shows the MSE of the time domain channel estimation versus SNR under different oversampling rates. As 


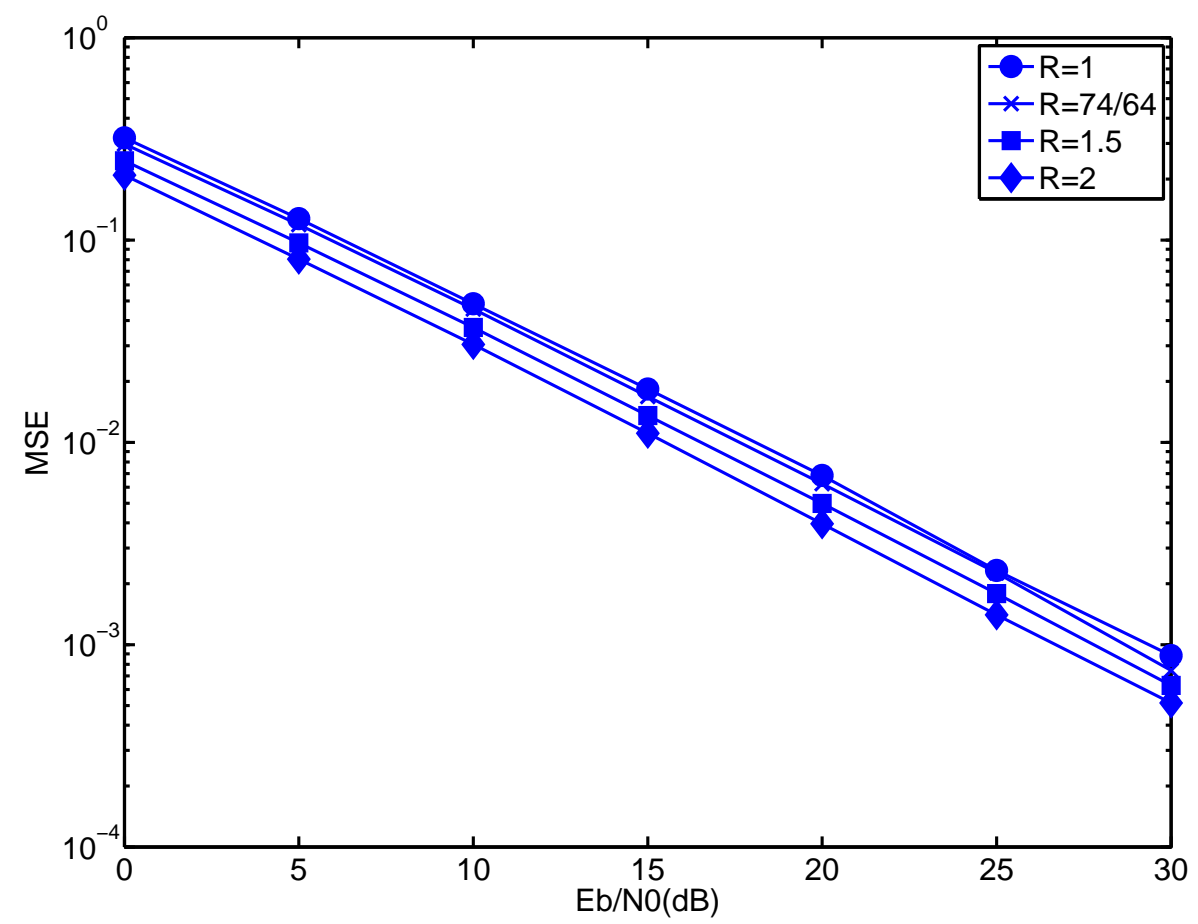

Figure 15: MSEs of time-domain channel estimation with different oversampling rates

expected, the MSE decreases with both $E_{b} / N_{0}$ and $R$ increasing. Furthermore, Figure 16 illustrates the MSE of the channel estimation versus the oversampling rate under four specific SNR values $(5 \mathrm{~dB}, 10 \mathrm{~dB}, 15 \mathrm{~dB}$ and $20 \mathrm{~dB})$. It can be seen that, comparing the MSE of $R=10$ with that of $R=1$, the performance gain of channel estimation using FDO is about $5 \mathrm{~dB}$. On the other hand, the performance improvement is subject to diminishing return for all SNR values, where the gain is more notable when the oversampling rate increases from 1 to 4 .

Finally, Figure 17 shows the BER comparison of the proposed FSFD MMSE receiver with conventional SC-FDE under different channel estimations, where $R_{a}$ and $R_{b}$ represent the oversampling rate of the channel equalization and estimation, respectively. The Rayleigh fading channel described in Section D.2 is adopted. Note that the BER curves for $R_{a}=1$ and $R_{a}=2$ with perfect CSI are also plotted as performance upper 


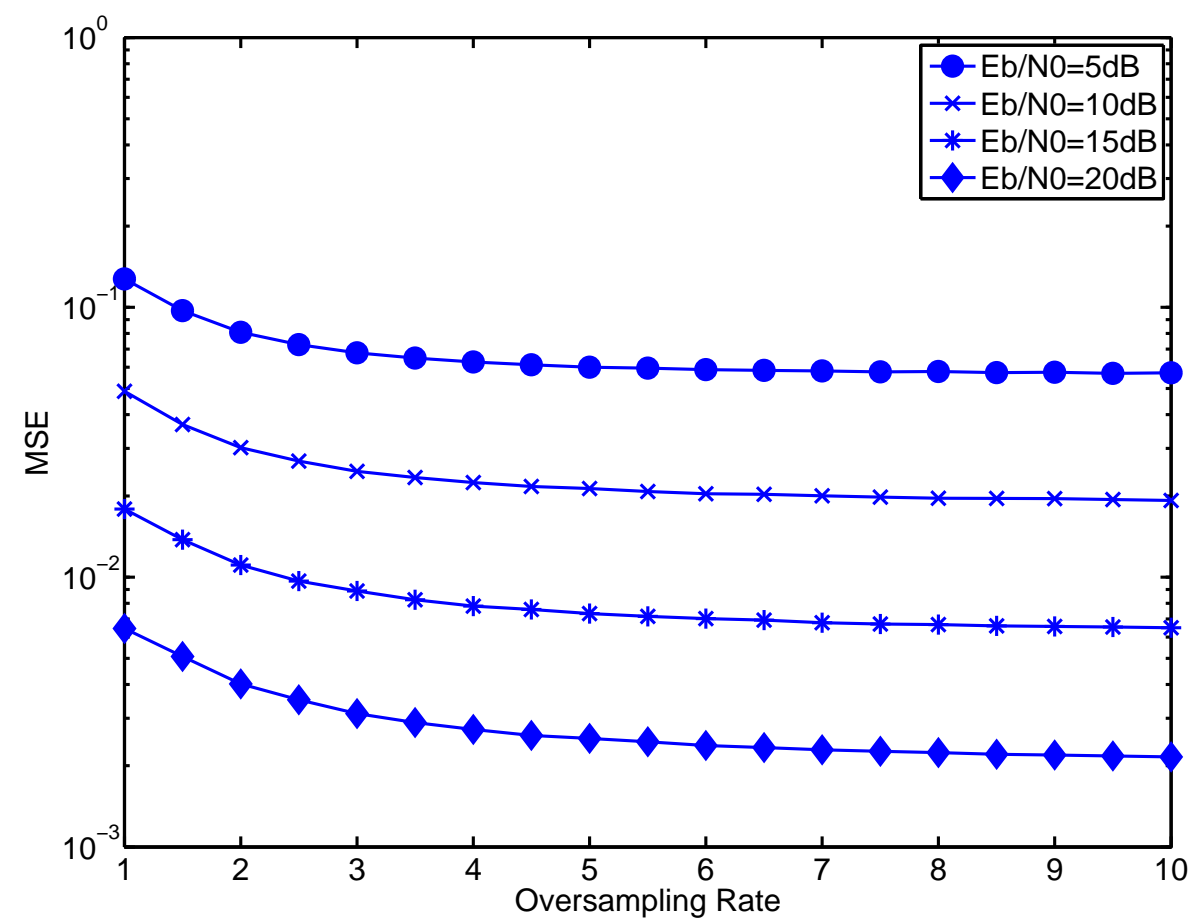

Figure 16: MSEs of time-domain channel estimation versus oversampling rate $\mathrm{R}$

bounds. It shows that, with the same $R_{a}$, the BER performance increases with $R_{b}$, which demonstrates the effectiveness of the FDO in channel estimation. On the other hand, with the same $R_{b}$, the BER performance increases with $R_{a}$, which shows the advantage of FDO in channel equalization with imperfect CSI.

These results confirm that both the channel estimation and channel equalization of SC-FDE can benefit from FDO. For equalization, FDO can improve the performance of SC-FDE under different channel models. The performance gain from FDO is larger as the frequency selectivity increases. For channel estimation, FDO can improve the estimation acuracy when the training sequence is not ideal (i.e., strong fluctuation in frequency domain). Therefore, FDO can further extract the frequency diversity and give robustness against frequency-domain deep fading and unideal training sequences. 


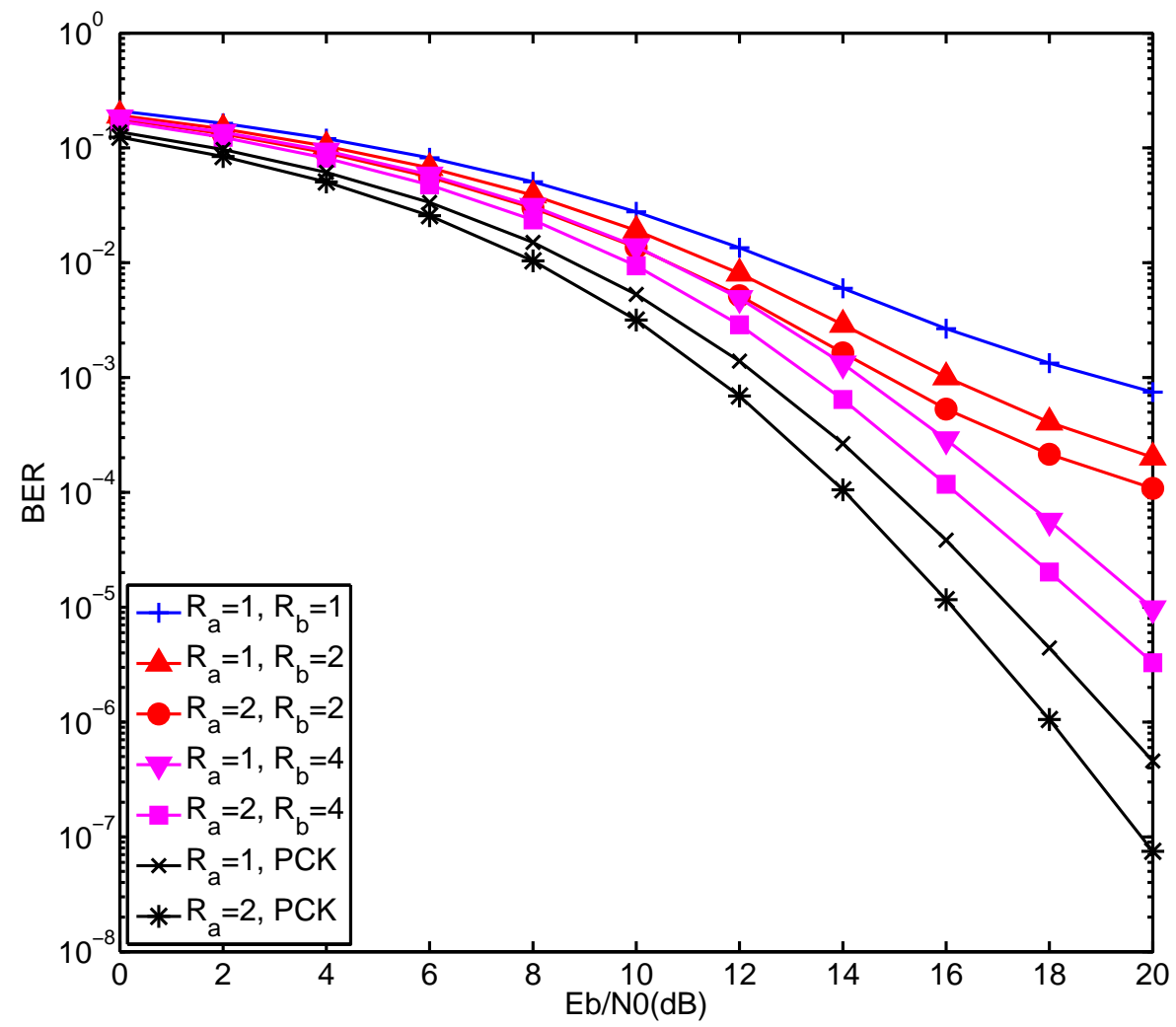

Figure 17: BER of MMSE receivers with different oversampling rates for channel estimation and equalization. 


\section{CHAPTER III}

\section{ITERATIVE IQ IMBALANCE COMPENSATION RECEIVER FOR SC-FDE}

With the increasing demand for high data rate wireless services, low cost transceivers becomes increasingly attractive for the implementation of wireless communication systems. However, such implementation is quite challenging due to the impairments caused by imperfections of the radio frequency (RF) front-end. For instance, OFDM, the widely used technique in high speed wireless communication over frequency-selective channel, suffers from the non-linearity of RF power amplifier because of its high peak-to-average power ratio (PAPR). Hence, single carrier block transmission has been developed as an alternative technique for broadband wireless systems to mitigate ISI [2][30]. By avoiding the OFDM drawback of high PAPR [15][16], SC transmission has been adopted by wireless standards (e.g., IEEE 802.11ad [31] and IEEE 802.15.3c [32]) to support low power and low complexity wireless transmission. In particular, the hybrid approach (OFDM for downlink and SC block transmission for uplink) has been adopted by the Third Generation Partnership Project (3GPP) Long Term Evolution (LTE) standard [17].

In addition to the non-linearity of RF power amplifier, IQ imbalance is another major issue that needs to be considered in low cost architectures, such as direct conversion 
transmitter (DCT) [33]. IQ imbalance may occur during up-convertor at the transmitter and down-convertor at the receiver because of the mismatch of the two branches. The distortion introduced by IQ imbalance can be frequency-independent (caused by the amplitude and phase difference in the non-ideal mixer) or frequency-dependent (caused by inaccuracy of the branch filters). Without proper compensation techniques, the transmission capability of wireless system under IQ imbalance will be seriously degraded. In this chapter, an intervene IQ imbalance compensation method will be derived to mitigate the effect of IQ imbalance in SC-FDE.

\section{A Background}

In general, it is difficult to compensate the IQ imbalance in analog domain due to its cost and inefficiency [34]. Consequently, many related works have focused on mitigating the effect of IQ imbalance in digital baseband. For OFDM systems, the impact of IQ imbalance is studied in [35] [36]. Furthermore, a closed-form bit error rate (BER) analysis of OFDM system under IQ imbalance is derived in [37]. These results show that the effect of IQ imbalance produces so called mirror carrier interference (MCI) causing the loss of orthogonality among the subcarriers. Various IQ imbalance estimation and compensation schemes have been proposed for the compensation of frequency-independent [38] and frequency-dependent IQ imbalance [34]. Initially, most solutions use linear compensation methods, such as Least Square (LS) [39] and Minimum Mean Square Error (MMSE) [40]. Later nonlinear receivers like Derision Direct [41], Expectation Maximization (EM) [42] and Maximum Likelihood (ML) [43] are introduced for better performance. 
Despite these studies, few works can be found in the literature on IQ imbalance in SC-FDE [44-49]. In fact, performance comparison between OFDM and SC-FDE under IQ imbalance shows that SC-FDE has slightly better robustness against IQ imbalance, but till suffers serious performance degradation [46]. Under frequency-independent IQ imbalance, the training sequence design for LS channel estimation of SC-FDE is studied in [44]. Then an enhanced channel estimation is proposed using Alternative LS (ALS) algorithm [45]. Furthermore, [47] derives the channel model of SC-FDE with independent Tx/Rx frequency IQ imbalance, PN sequence aided channel estimation and MMSE compensation. Considering the presence of both IQ imbalance and carrier frequency offset, a three stage compensation scheme for SC-FDE is provided in [48]. For millimeter-wave SC-FDE systems, Cheng X. et.al. [49] propose a joint channel and IQ imbalance estimation method using ML criterion and IQ imbalance compensation using MMSE criterion. However, most existing works focus on the channel estimation under frequency-independent IQ imbalance and use linear compensation methods to eliminate the effect of IQ imbalance. Although linear receivers benefit from their low complexity, the BER performance is far from the match filter bound (MFB), especially in highly frequency-selective wireless channel [50]. There exist several nonlinear receivers for SC-FDE system without IQ imbalance. Among them, one promising design is the iterative block decision feedback equalization (IBDFE) [51], which employs feedforward and feedback filters in frequency domain and can achieve BER performance that is close to the MFB. Its iterative architecture has been extended to several special scenarios, such as interference channels [50] and system with imperfect channel state information (CSI) [52]. 
With imperfect RF, conventional IBDFE receiver may suffer serious performance degradation due to the MCI generated by transceiver IQ imbalance. To overcome this, the joint effects of IQ imbalance and multipath channel are required to mitigate together in the iterative decision feedback architecture design. In this chapter, it will derive the architecture of iterative decision feedback receiver for SC-FDE with IQ imbalance, where transmitter (Tx) and receiver ( $\mathrm{Rx}$ ) frequency-dependent IQ imbalance are jointly considered with the wireless channel. Unlike OFDM, in SC-FDE both Discrete Fourier Transform (DFT) and inverse DFT (IDFT) are located at the receiver-side. As a result, the interference suppression design on two self-mirror subcarriers is also carefully formulated, while OFDM system may choose not to carry information symbols on those two subcarriers [39]. Furthermore, feedforward and feedback filters are introduced to mitigate the MCI in frequency domain. Both hard detection and soft detection based feedback schemes are designed to combat the joint effect of IQ imbalance and wireless multipath channel. Also, In order to show the effectiveness of proposed receiver under imperfect CSI, pilot-aided channel estimation methods performing separately in frequency and time domains are also presented to estimate the CSI. Simulation results show that the proposed receiver outperforms the MMSE IQ imbalance compensation and can achieve similar performance with conventional IBDFE receiver under ideal RF.

The notations used in this chapter are given as follows: $(\cdot)^{*}$ and $(\cdot)^{H}$ denote the conjugate and conjugate transpose, respectively; $\mathbf{I}$ is the $2 \times 2$ identity matrix; $\mathrm{E}\{\cdot\}$ is the expectation operation; $\operatorname{tr}(\cdot)$ represents the trace of a matrix; $\Re\{\cdot\}$ and $\Im\{\cdot\}$ are the corresponding real and imaginary parts of a complex number or vector; the $N$-point circular convolution are denoted by $\otimes$. 


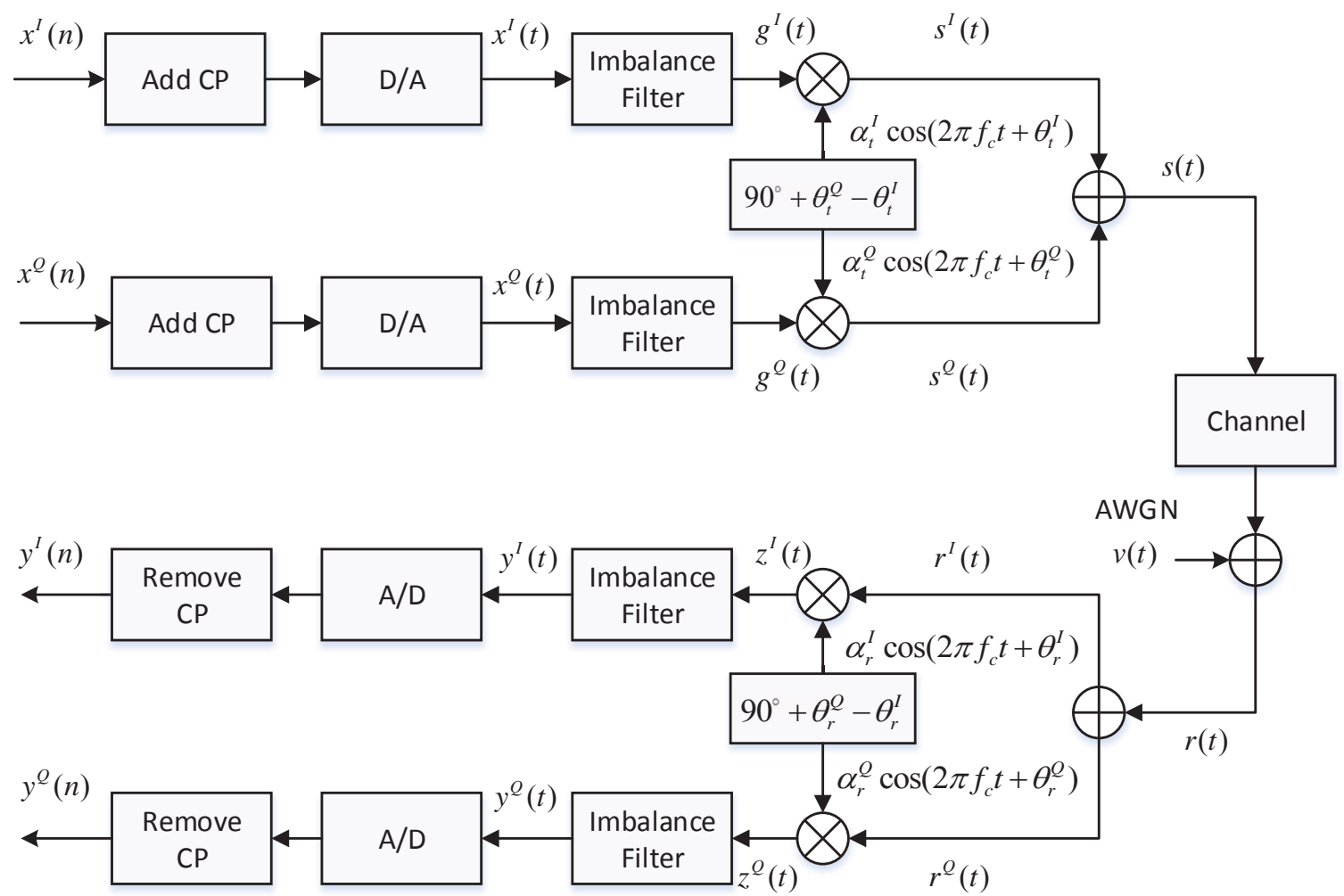

Figure 18: Block diagram of transceiver for single carrier transmission with IQ imbalance.

The remainder of this chapter is organized as follows. Section II describes the system model of SC-FDE with Tx/Rx frequency-dependent IQ imbalance and derive its frequency domain representation. Section III designs the iterative decision feedback receiver for IQ imbalance compensation and provides the estimation methods for the feedforward and feedback filters design under both hard detection and soft detection. Section IV discusses channel estimation in SC-FDE with IQ imbalance, followed by numerical results in Section V. 


\section{B System model}

This section provides the description of the system model of the single carrier transmission under Tx/Rx frequency-dependent IQ imbalance.

\section{Single Carrier Block Transmission With IQ Imbalance}

The transceiver diagram is given in Figure 18. Let $N$ be the block length and $x(n)=x^{I}(n)+j \cdot x^{Q}(n), n \in\{1,2, \cdots, N\}$, be the $n^{\text {th }}$ symbol to be transmitted with average power $\sigma_{x}^{2}$. The complex digital baseband signal is first inserted with cyclic prefix (CP) to avoid inter-block interference. After that, the digital signal is transformed into analog form by a digital-analog convertor (DAC). Let $x(t)=x^{I}(t)+j \cdot x^{Q}(t)$ denote the DAC output signal, where $x^{I}(t)$ and $x^{Q}(t)$ are the corresponding signals of in-phase (I) and quadrature $(\mathrm{Q})$ branches. The baseband signal $x(t)$ is up-converted and transmitted by the RF frontend. Due to the imperfection of RF frontend, the signal is distorted before travailing thorough the wireless channel. The distortion, known as transmitter IQ imbalance, is caused by two factors: (1) the mismatch of IQ branch filters which yield frequency-dependent IQ imbalance. (2) amplitude imbalance and phase difference of the mixer that produce frequency-independent IQ imbalance.

Here, the mismatch of transmitter's I and Q branches is modeled as two filters $\xi_{t}^{I}(t)$ and $\xi_{t}^{Q}(t)$, respectively. The amplitude and phase imbalance of the mixer are denoted as $\alpha_{t}^{I}, \alpha_{t}^{Q}$ and $\theta_{t}^{I}, \theta_{t}^{Q}$, receptively. After passing through imbalance filters, the distorted signal before the mixer is formulated by

$$
g(t)=g^{I}(t)+j \cdot g^{Q}(t)
$$


with

$$
\begin{gathered}
g^{I}(t)=x^{I}(t) \otimes \xi_{t}^{I}(t), \\
g^{Q}(t)=x^{Q}(t) \otimes \xi_{t}^{Q}(t) .
\end{gathered}
$$

After up-conversion, the passband signal is defined by

$$
\begin{aligned}
s_{p}(t) & =g^{I}(t) \alpha_{t}^{I} \cos \left(2 \pi f_{c} t+\theta_{t}^{I}\right)-g^{Q}(t) \alpha_{t}^{Q} \sin \left(2 \pi f_{c} t+\theta_{t}^{Q}\right) \\
& =s^{I}(t) \cos \left(2 \pi f_{c} t\right)-s^{Q}(t) \sin \left(2 \pi f_{c} t\right),
\end{aligned}
$$

in which $s^{I}(t)$ and $s^{Q}(t)$ representing the corresponding IQ baseband signals are given by

$$
\begin{aligned}
& s^{I}(t)=g^{I}(t) \alpha_{t}^{I} \cos \left(\theta_{t}^{I}\right)-g^{Q}(t) \alpha_{t}^{Q} \sin \left(\theta_{t}^{Q}\right), \\
& s^{Q}(t)=g^{Q}(t) \alpha_{t}^{Q} \cos \left(\theta_{t}^{Q}\right)-g^{I}(t) \alpha_{t}^{I} \sin \left(\theta_{t}^{I}\right) .
\end{aligned}
$$

Substituting $g^{I}(t)=\left(g(t)+g^{*}(t)\right) /(2)$ and $g^{Q}(t)=\left(g(t)-g^{*}(t)\right) /(2 j)$ into (58) and (59) and grouping the terms carefully, the baseband equivalent signal after mixer can be expresses as

$$
s(t)=\mu_{t} g(t)+\nu_{t} g^{*}(t)
$$

with $\mu_{t}=(1 / 2)\left(\alpha_{t}^{I} e^{j \theta_{t}^{I}}+\alpha_{t}^{Q} e^{j \theta_{t}^{Q}}\right)$ and $\nu_{t}=(1 / 2)\left(\alpha_{t}^{I} e^{j \theta_{t}^{I}}-\alpha_{t}^{Q} e^{j \theta_{t}^{Q}}\right)$. Substituting (54) into (60) and use the relation $x_{I}(t)=\left(x(t)+x^{*}(t)\right) /(2)$ and $x_{Q}(t)=\left(x(t)-x^{*}(t)\right) /(2 j)$, it has

$$
s(t)=\lambda_{t}(t) \otimes x(t)+\phi_{t}(t) \otimes x^{*}(t),
$$

in which

$$
\begin{aligned}
& \lambda_{t}(t)=\frac{1}{2}\left(\left(\mu_{t}+\nu_{t}^{*}\right) \xi_{t}^{I}(t)+\left(\mu_{t}-\nu_{t}^{*}\right) \xi_{t}^{Q}(t)\right), \\
& \phi_{t}(t)=\frac{1}{2}\left(\left(\mu_{t}+\nu_{r}^{*}\right) \xi_{t}^{I}(t)-\left(\mu_{t}-\nu_{t}^{*}\right) \xi_{t}^{Q}(t)\right) .
\end{aligned}
$$


Then the transmitted signal goes through the wireless channel. At the receiver-side, the equivalent baseband signal after the down-conversion is given by

$$
r(t)=h(t) \otimes s(t)+v(t)
$$

where $h(t)$ is the channel impulse response and $v(t)$ is the additive white Gaussian noise (AWGN) with power spectral density of $\sigma_{v}^{2}$. Let $\alpha_{r}^{I}, \alpha_{r}^{Q}$ and $\theta_{r}^{I}, \theta_{r}^{Q}$ denote the amplitude and the phase imbalance of the receiver-side mixer, respectively. The mixer output is given by

$$
z(t)=\frac{1}{2}\left(\mu_{r} r(t)+\nu_{r} r^{*}(t)\right)
$$

where $\mu_{r}=(1 / 2)\left(\alpha_{r}^{I} e^{-j \theta_{r}^{I}}+\alpha_{r}^{Q} e^{-j \theta_{r}^{Q}}\right)$ and $\nu_{r}=(1 / 2)\left(\alpha_{r}^{I} e^{j \theta_{r}^{I}}-\alpha_{r}^{Q} e^{j \theta_{r}^{Q}}\right)$. The mismatch of receiver branch filters are denoted as $\xi_{r}^{I}(t)$ for in-phase and $\xi_{r}^{Q}(t)$ for quadrature phase. The output of receiver-side branch filters is given by

$$
y(t)=\lambda_{r}(t) \otimes z(t)+\phi_{r}(t) \otimes z^{*}(t)
$$

with

$$
\begin{aligned}
& \lambda_{r}(t)=\frac{1}{2}\left(\left(\mu_{r}+\nu_{r}^{*}\right) \xi_{r}^{I}(t)+\left(\mu_{r}-\nu_{r}^{*}\right) \xi_{r}^{Q}(t)\right), \\
& \phi_{r}(t)=\frac{1}{2}\left(\left(\nu_{r}+\mu_{r}^{*}\right) \xi_{r}^{I}(t)+\left(\nu_{r}-\mu_{r}^{*}\right) \xi_{r}^{Q}(t)\right) .
\end{aligned}
$$

Form (61), (64), (65) and (66), the analog input-output relation of the system can be rewritten as:

$$
y(t)=\psi_{1}(t) \otimes x(t)+\psi_{2}(t) \otimes x^{*}(t)+\bar{v}(t),
$$

where

$$
\psi_{1}(t)=\lambda_{r}(t) \otimes h(t) \otimes \lambda_{t}(t)+\phi_{r}(t) \otimes h^{*}(t) \otimes \phi_{t}^{*}(t)
$$




$$
\begin{gathered}
\psi_{2}(t)=\lambda_{r}(t) \otimes h(t) \otimes \phi_{t}(t)+\phi_{r}(t) \otimes h^{*}(t) \otimes \lambda_{t}^{*}(t), \\
\bar{v}(t)=\lambda_{r}(t) \otimes v(t)+\phi_{r}(t) \otimes v^{*}(t) .
\end{gathered}
$$

From (69), it can be observed that $\psi_{1}(n)$ and $\psi_{2}(n)$ represent the overall effect of transceiver IQ imbalance and wireless channel on the transmitted signal. While $\psi_{1}(n)$ combines the original transmitted signal, $\psi_{2}(n)$ introduces interference in the form of a conjugated signal. The input-output relation given in (69) is a simplified model of [34], since it only considers the time invariant channel and ignores the effect of ideal low-pass filter at receiver side (The ideal low-pass filter in [34] does not contribute to the system I/Q imbalance and can be jointly considered with the wireless channel).

Assume that the CP length is larger than the memory lengths of $\psi_{1}(n)$ and $\psi_{2}(n)$ defined in (70) and (71). After analog-to-digital conversion (ADC) and the CP exclusion, denote $y(n)=y(n T), 1 \leq n \leq N$, as the sampling of $y(t)$ in the current block, where $T$ is the sampling period. The corresponding discrete input-output relation in time domain is obtained as

$$
y(n)=\psi_{1}(n) \circledast x(n)+\psi_{2}(n) \circledast x^{*}(n)+\bar{v}(n),
$$

in which

$$
\begin{gathered}
\psi_{1}(n)=\lambda_{r}(n) \circledast h(n) \circledast \lambda_{t}(n)+\phi_{r}(n) \circledast h^{*}(n) \phi_{t}^{*}(n), \\
\psi_{2}(n)=\lambda_{r}(n) \circledast h(n) \circledast \phi_{t}(n)+\phi_{r}(n) \circledast h^{*}(n) \circledast \lambda_{t}^{*}(n), \\
\bar{v}(n)=\lambda_{r}(n) \circledast v(n)+\phi_{r}(n) \circledast v^{*}(n),
\end{gathered}
$$

and $\psi_{1}(n), \psi_{2}(n), \lambda_{r}(n), \lambda_{t}(n), h(n), \phi_{t}(n), \phi_{r}(n)$ are the samples of $\psi_{1}(t), \psi_{2}(t), \lambda_{r}(t)$, $\lambda_{t}, h(t), \phi_{t}(t), \phi_{r}(t)$, respectively. 
Let $\mathbf{y}=[y(1), y(2), \cdots, y(N)]^{T}, \mathbf{x}=[x(1), x(2), \cdots, x(N)]^{T}$ and $\overline{\boldsymbol{v}}=[\bar{v}(1)$, $\overline{v(2)}, \cdots, \bar{v}(N)]^{T}$ represent the corresponding signal and noise vectors. Denote $\boldsymbol{\psi}_{1}$ and $\boldsymbol{\psi}_{2}$ as $N \times 1$ vectors with first $L$ elements respectively being $\psi_{1}(l)$ and $\psi_{2}(l)(1 \leq l \leq L)$, and the rest being zeros, where $L$ is the length of channel impulse response $\psi_{1}(n)$ and $\psi_{2}(n)$. Likewise, it has $N \times 1$ vectors $\boldsymbol{\lambda}_{t}, \boldsymbol{\lambda}_{r}, \mathbf{h}, \boldsymbol{\phi}_{t}, \boldsymbol{\phi}_{r}$ associated with $\lambda_{t}(n), \lambda_{r}(n), h(n), \phi_{t}(n)$, $\phi_{r}(n)$, respectively. Then the input-output relation in (73) can be rewritten in a matrix form as

$$
\mathbf{y}=\Psi_{1} \mathbf{x}+\Psi_{2} \mathbf{x}^{*}+\overline{\boldsymbol{v}}
$$

in which

$$
\begin{aligned}
& \boldsymbol{\Psi}_{1}=\boldsymbol{\Lambda}_{r} \mathbf{H} \boldsymbol{\Lambda}_{t}+\boldsymbol{\Phi}_{r} \mathbf{H}^{*} \boldsymbol{\Phi}_{t}^{*} \\
& \boldsymbol{\Psi}_{2}=\boldsymbol{\Lambda}_{r} \mathbf{H} \boldsymbol{\Phi}_{t}+\boldsymbol{\Phi}_{r} \mathbf{H}^{*} \boldsymbol{\Lambda}_{t}^{*},
\end{aligned}
$$

and $\boldsymbol{\Lambda}_{t}, \boldsymbol{\Lambda}_{r}, \mathbf{H}, \boldsymbol{\Phi}_{t}, \boldsymbol{\Phi}_{r}$ are $N \times N$ circular matrices consisting of $\boldsymbol{\lambda}_{t}, \boldsymbol{\lambda}_{r}, \mathbf{h}, \boldsymbol{\phi}_{t}, \boldsymbol{\phi}_{r}$, respectively. As a result, $\Psi_{1}$ and $\Psi_{2}$ are also circular with first column being $\boldsymbol{\psi}_{1}$ and $\boldsymbol{\psi}_{2}$, respectively.

\section{Frequency Domain Representation}

This section derives the system transceiver relation in frequency domain. Let $\mathbf{F}$ be the DFT matrix of size $N$. Since both $\Psi_{1}$ and $\Psi_{2}$ can be diagonalized by the DFT matrix, diagonal matrices $\Gamma_{1}=\mathbf{F} \Psi_{1} \mathbf{F}^{H}$ and $\Gamma_{2}=\mathbf{F} \Psi_{2} \mathbf{F}^{H}$ are the corresponding frequency domain channel matrices of the system. Taking the DFT of the time domain signal in (77), the received signal in frequency domain becomes

$$
\mathbf{Y}=\boldsymbol{\Gamma}_{1} \mathbf{X}+\boldsymbol{\Gamma}_{2} \mathbf{X}^{\#}+\mathbf{V}
$$


with $\mathbf{Y}=\mathbf{F y}, \mathbf{X}=\mathbf{F x}, \mathbf{V}=\mathbf{F} \overline{\mathbf{v}}$ and $\mathbf{X}^{\#}=\mathbf{F x}^{*}$ being the DFT of corresponding terms.

$$
\begin{gathered}
x(n) \stackrel{\text { DFT }}{\longrightarrow} X(k) \\
x^{*}(n) \stackrel{\text { DFT }}{\longrightarrow} X^{*}(N-k-2)
\end{gathered}
$$

Using the well known DFT property described in (81) and (82), the mapping from $\mathbf{X}$ to $\mathrm{X}^{\#}$ can be written as follows

$$
\mathbf{X}=\left[\begin{array}{c}
X(1) \\
X(2) \\
\vdots \\
X(N / 2) \\
X(N / 2+1) \\
X(N / 2+2) \\
\vdots \\
X(N)
\end{array}\right] \Longrightarrow \mathbf{X}^{\#}=\left[\begin{array}{c}
X^{*}(1) \\
X^{*}(N) \\
\vdots \\
X^{*}(N / 2+2) \\
X^{*}(N / 2+1) \\
X^{*}(N / 2) \\
\vdots \\
X^{*}(2)
\end{array}\right]
$$

Let $\gamma_{1}(k)$ and $\gamma_{2}(k)$ be the $k^{\text {th }}$ diagonal element of the $\boldsymbol{\Gamma}_{1}$ and $\boldsymbol{\Gamma}_{2}$, respectively. It has $\gamma_{1}(k)=\operatorname{DFT}\left\{\psi_{1}(n)\right\}$ and $\gamma_{2}(k)=\operatorname{DFT}\left\{\psi_{2}(n)\right\}$. Then the $k^{\text {th }}$ element of frequency domain signal $\mathbf{Y}$ is obtained from (80) as

$$
Y(k)=\gamma_{1}(k) X(k)+\gamma_{2}(k) X^{*}(N-k+2)+V(k),
$$

where $X(k)$ and $V(k)$ are the $k^{t h}$ elements in $\mathbf{X}$ and V. Similarly, the conjugate of the $(N-k+2)^{t h}$ element of frequency domain signal $\mathrm{Y}$ is given by

$$
\begin{aligned}
& Y(N-k+2)^{*}=\gamma_{1}^{*}(N-k+2) X^{*}(N-k+2) \\
& +\gamma_{2}(N-k+2)^{*} X(k)+V(N-k+2) .
\end{aligned}
$$


For $k=2, \cdots, N / 2$, equation (84) and (85) can be rewritten in matrix form as

$$
\mathbf{Y}_{k}=\boldsymbol{C}_{k} \mathbf{X}_{k}+\mathbf{V}_{k}, \quad k=2, \cdots, \frac{N}{2}
$$

where

$$
\begin{aligned}
& \mathbf{Y}_{k}= {\left[\begin{array}{c}
Y(k) \\
Y^{*}(N-k+2)
\end{array}\right], \quad k=2, \cdots, \frac{N}{2}, } \\
& \mathbf{X}_{k}= {\left[\begin{array}{c}
X(k) \\
X^{*}(N-k+2)
\end{array}\right], \quad k=2, \cdots, \frac{N}{2}, } \\
& \mathbf{V}_{k}= {\left[\begin{array}{c}
V(k) \\
V^{*}(N-k+2)
\end{array}\right], \quad k=2, \cdots, \frac{N}{2}, } \\
& \boldsymbol{C}_{k}= {\left[\begin{array}{c}
\gamma_{1}(k) \\
\gamma_{2}^{*}(N-k+2) \\
\gamma_{1}^{*}(N-k+2)
\end{array}\right] } \\
& k=2, \cdots, \frac{N}{2} .
\end{aligned}
$$

When $k=1$ or $k=N / 2+1$, it has the following equation from (84) and (85):

$$
\begin{array}{r}
Y(k)=\gamma_{1}(k) X(k)+\gamma_{2}(k) X^{*}(k)+V(k), \\
k=1 \text { or } k=\frac{N}{2}+1 .
\end{array}
$$

From equation (91), it can be seen that the subcarriers with index $k=1$ or $k=N / 2+1$ mirror to themselves. Because of the conjugated term in it, $X(k)$ can not be solved directly for $k=1$ or $k=N / 2+1$. In OFDM, it can choose not to carry information symbols on those two subcarriers [39], while in SC-FDE all subcarriers carry information because both DFT and IDFT are operated at the transmitter. Thus, frequency domain signals on 
those two subcarriers are also required to be recovered. By carefully decoupling the real and imaginary parts of corresponding terms, it obtains the following matrix form of (91) as

$$
\mathbf{Y}_{k}=C_{k} \mathbf{X}_{k}+\mathbf{V}_{k}, \quad k=1 \text { or } k=\frac{N}{2}+1,
$$

where

$$
\begin{gathered}
\mathbf{Y}_{k}=\left[\begin{array}{l}
\Re\{Y(k)\} \\
\Im\{Y(k)\}
\end{array}\right], \quad k=1 \text { or } k=\frac{N}{2}+1, \\
\mathbf{X}_{k}=\left[\begin{array}{l}
\Re\{X(k)\} \\
\Im\{X(k)\}
\end{array}\right], \quad k=1 \text { or } k=\frac{N}{2}+1, \\
\mathbf{V}_{k}=\left[\begin{array}{l}
\Re\{V(k)\} \\
\Im\{V(k)\}
\end{array}\right], \quad k=1 \text { or } k=\frac{N}{2}+1, \\
\boldsymbol{C}_{k}=\left[\begin{array}{ll}
\Re\left\{\gamma_{1}(k)+\gamma_{2}(k)\right\} & \Im\left\{\gamma_{2}(k)-\gamma_{1}(k)\right\} \\
\Im\left\{\gamma_{1}(k)+\gamma_{2}(k)\right\} & \Re\left\{\gamma_{1}(k)-\gamma_{2}(k)\right\}
\end{array}\right], \\
\quad k=1 \text { or } k=\frac{N}{2}+1 .
\end{gathered}
$$

According to (86) and (92), the effect of Tx/Rx IQ imbalance and multipath channel can be jointly compensated in frequency domain, provided the receiver has the frequency domain CSI $\boldsymbol{C}_{k}(1 \leq k \leq N / 2+1)$.

\section{Iterative IQ Imbalance Compensation}

In this section, it derives an iterative receiver for single carrier system to jointly compensate the IQ imbalance caused by RF imperfection of both transmitter and receiver. 


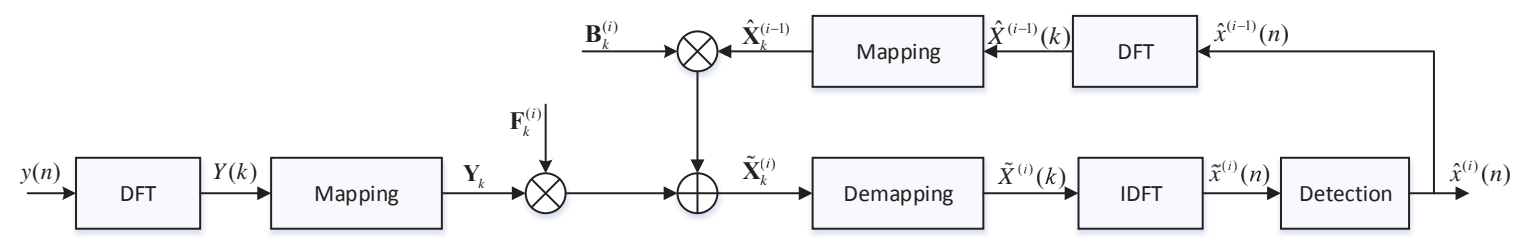

Figure 19: Block diagram of iterative IQ imbalance compensation receiver.

1 Iterative Receiver Architecture

The iterative IQ imbalance compensation receiver is a direct extension of the IBDFE proposed in [51] for single carrier systems under IQ imbalance. As shown in Figure 19, the output frequency domain data of proposed receiver at iteration $i$ can be expressed as

$$
\tilde{\mathbf{X}}_{k}^{(i)}=\mathbf{F}_{k}^{(i)} \mathbf{Y}_{k}+\mathbf{B}_{k}^{(i)} \hat{\mathbf{X}}_{k}^{(i-1)}, \quad k=1,2 \cdots, N / 2+1,
$$

where $\mathbf{F}_{k}^{(i)}$ and $\mathbf{B}_{k}^{(i)}$ are both $2 \times 2$ matrices representing the feedforward and feedback coefficients at iteration $i$, respectively; $\hat{\mathbf{X}}_{k}^{(i-1)}$ is the feedback signal generated by taking the DFT of the detected data $\hat{\mathrm{x}}^{i-1}$ at previous iteration followed by a mapping operation defined in (88) and (94). The goal of feedforward and feedback coefficients design is to jointly compensate the effect of IQ imbalance and multipath channel by minimizing the mean square error (MSE) between the estimated symbol and the transmitted symbol at the detection point of the receiver. The MSE at iteration $i$ is defined by

$$
\begin{aligned}
\operatorname{MSE}^{(i)} & =\frac{1}{N} \sum_{n=1}^{N} \mathrm{E}\left|\tilde{x}(n)^{(i)}-x(n)\right|^{2} \\
& =\frac{1}{N} \sum_{k=1}^{N} \mathrm{E}\left|\tilde{X}(k)^{(i)}-X(k)\right|^{2} \\
& =\frac{1}{N} \sum_{k=1}^{N / 2+1} \mathrm{E}\left\{\operatorname{tr}\left[\left(\tilde{\mathbf{X}}_{k}^{(i)}-\mathbf{X}_{k}\right)\left(\tilde{\mathbf{X}}_{k}^{(i)}-\mathbf{X}_{k}\right)^{H}\right]\right\} .
\end{aligned}
$$

Assume that the transmitted signal, noise and detected signal have independent identical distribution (i.i.d.). Denote the power of frequency domain transmitted data, 
noise and detected data at iteration $i$ as $P_{x}=\mathrm{E}\left\{X(k) X^{*}(k)\right\}, P_{v}=\mathrm{E}\left\{V(k) V^{*}(k)\right\}$ and $P_{\hat{x}}^{(i)}=\mathrm{E}\left\{\hat{X}^{(i)}(k) \hat{X}^{(i) *}(k)\right\}$, respectively. The cross-correlation of transmitted data and detected data at iteration $i$ is denoted by

$$
R_{\hat{x} x}^{(i)}=\mathrm{E}\left\{X(k) \hat{X}^{(i) *}(k)\right\} .
$$

Note that these parameters are not dependent on subcarrier index $k$. Then the covariance matrices of corresponding signals after mapping are given as

$$
\begin{gathered}
\mathrm{E}\left\{\mathbf{X}_{k} \mathbf{X}_{k}^{H}\right\}=P_{x} \mathbf{I}, \\
\mathrm{E}\left\{\mathbf{V}_{k} \mathbf{V}_{k}^{H}\right\}=P_{v} \mathbf{I}, \\
\mathrm{E}\left\{\mathbf{X}_{k} \hat{\mathbf{X}}_{k}^{(i) H}\right\}=R_{\hat{x} x}^{(i)} \mathbf{I}, \\
\mathrm{E}\left\{\hat{\mathbf{X}}_{k}^{(i)} \hat{\mathbf{X}}_{k}^{(i) H}\right\}=P_{\hat{x}}^{(i)} \mathbf{I} .
\end{gathered}
$$

Substituting (97) into (98) and taking the expectation in it with respect to $\mathbf{X}_{k}$ and $\mathrm{V}_{k}$, the MSE in (98) can be rewritten as

$$
\begin{aligned}
\operatorname{MSE}^{(i)}= & \frac{1}{N} \sum_{k=1}^{N / 2+1} \operatorname{tr}\left[\left(\mathbf{I}-\mathbf{F}_{k}^{(i)} \mathbf{C}_{k}\right)\left(\mathbf{I}-\mathbf{F}_{k}^{(i)} \mathbf{C}_{k}\right)^{H}\right] P_{x} \\
& -2 \Re\left\{\operatorname{tr}\left[\left(\mathbf{I}-\mathbf{F}_{k}^{(i)} \mathbf{C}_{k}\right) \mathbf{B}_{k}^{(i) H}\right] R_{\hat{x} x}^{(i-1)}\right\} \\
& +\operatorname{tr}\left[\mathbf{F}_{k}^{(i)} \mathbf{F}_{k}^{(i) H}\right] P_{v}+\operatorname{tr}\left[\mathbf{B}_{k}^{(i)} \mathbf{B}_{k}^{(i) H}\right] P_{\hat{x}}^{(i-1)}
\end{aligned}
$$

Note that the diagonal elements of feedback filter $\mathbf{B}_{k}^{(i)}$ are associated with the desired signal, while the elements in the anti-diagonal $\mathbf{B}_{k}^{(i)}$ are related to the interference caused by IQ imbalance. For iterative receiver design, the following constraint is imposed, so that the feedback coefficients do not remove the desired signal component:

$$
\sum_{k=1}^{N / 2+1} \operatorname{tr}\left[\mathbf{B}_{k}^{(i)}\right]=0
$$


This constraint is similar to the one used in the design of conventional IBDFE receiver [51]. Note that the feedback is used to reconstruct and cancel the residual interference, which includes the mirror carrier interference caused by IQ imbalance and inter-symbol interference caused by multipath channel. The purpose of this constraint is to avoid self-subtraction of the desired signal by the previous estimation. A minor difference is that, in the presence of IQ imbalance, the feedback filter $\mathbf{B}_{k}^{(i)}$ is in matrix form, where the diagonal elements are associated with the desired signal, while the anti-diagonal elements are related to the mirror carrier interference. As a result, the term $\sum_{1}^{N / 2+1} \operatorname{tr}\left[\mathbf{B}_{k}^{(i)}\right]$ only add the diagonal elements.

Accordingly, the feedforward and feedback coefficients matrices $\mathbf{F}_{k}^{(i)}, \mathbf{B}_{k}^{(i)}$ can be found by minimizing the MSE in (104) under constraint (105). For such purpose, the following Lagrange function is constructed:

$$
J\left(\mathbf{F}_{k}^{(i)}, \mathbf{B}_{k}^{(i)}, \eta^{(i)}\right)=\operatorname{MSE}^{(i)}+\eta^{(i)} \sum_{k=1}^{N / 2+1} \operatorname{tr}\left[\mathbf{B}_{k}^{(i)}\right]
$$

in which $\eta^{(i)}$ is the non-negative Lagrange multiplier. Letting the partial derivatives of (106) with respect to $\mathbf{F}_{k}^{(i)}, \mathbf{B}_{k}^{(i)}$ and $\eta^{(i)}$ be zeros, it has

$$
\begin{gathered}
\frac{\partial J\left(\mathbf{F}_{k}^{(i)}, \mathbf{B}_{k}^{(i)}, \eta^{(i)}\right)}{\partial \mathbf{F}_{k}^{(i) *}=} 2 P_{x} \mathbf{F}_{k}^{(i)} \mathbf{C}_{k} \mathbf{C}_{k}^{H}-2 P_{x} \mathbf{C}_{k}^{H} \\
+2 P_{v} \mathbf{F}_{k}^{(i)}+2 R_{\hat{x} x}^{(i-1)} \mathbf{B}_{k}^{(i)} \mathbf{C}_{k}^{H}=0, \\
\frac{\partial J\left(\mathbf{F}_{k}^{(i)}, \mathbf{B}_{k}^{(i)}, \eta^{(i)}\right)}{\partial \mathbf{B}_{k}^{(i) *}}=-2 R_{\hat{x} x}^{(i-1)}\left(\mathbf{I}-\mathbf{F}_{k}^{(i)} \mathbf{C}_{k}\right) \\
+2 \mathbf{B}_{k}^{(i)} P_{\hat{x}}^{(i-1)}+\eta^{(i)} \mathbf{I}=0 \\
\frac{\partial J\left(\mathbf{F}_{k}^{(i)}, \mathbf{B}_{k}^{(i)}, \eta^{(i)}\right)}{\eta^{(i)}}=\sum_{k=1}^{N / 2+1} \operatorname{tr}\left[\mathbf{B}_{k}^{(i)}\right]=0 .
\end{gathered}
$$


First, the feedback coefficients matrix $\mathbf{B}_{k}^{(i)}$ is obtained by solving (108). After some manipulation, the feedback coefficients matrix $\mathbf{B}_{k}^{(i)}$ is given by

$$
\mathbf{B}_{k}^{(i)}=-\frac{R_{\hat{x} x}^{(i-1)}}{P_{\hat{x}}^{(i-1)}}\left(\mathbf{F}_{k}^{(i)} \mathbf{C}_{k}-\chi^{(i)} \mathbf{I}\right)
$$

where the introduced variable $\chi^{(i)}$ needs to be chosen properly, so that constraint (105) is satisfied. Plugging (110) into (105) gives

$$
\chi^{(i)}=\frac{1}{N+2} \sum_{k=1}^{N / 2+1} \operatorname{tr}\left[\mathbf{F}_{k}^{(i)} \mathbf{C}_{k}\right] .
$$

For the feedforward coefficients matrix $\mathbf{F}_{k}^{(i)}$ design, by inserting (110) into (107) and solving it with respect to $\mathbf{F}_{k}^{(i)}$, it gives

$$
\mathbf{F}_{k}^{(i)}=\mathbf{C}_{k}^{H}\left[\mathbf{C}_{k} \mathbf{C}_{k}^{H}\left(1-\beta^{(i-1)}\right)+\frac{P_{v}}{P_{x}} \mathbf{I}\right]^{-1}
$$

with

$$
\beta^{i-1}=\frac{\left|R_{\hat{x} x}^{(i-1)}\right|^{2}}{P_{x} P_{\hat{x}}^{(i-1)}} .
$$

When there is no prior knowledge on the data, the cross correlation $R_{\hat{x} x}^{(i-1)}$ equals zero and the feedback filter turns off. In this case, the proposed iterative decision feedback receiver is equivalent to the conventional MMSE IQ imbalance compensation [48]:

$$
\mathbf{F}_{k}^{(i)}=\mathbf{C}_{k}^{H}\left[\mathbf{C}_{k} \mathbf{C}_{k}^{H}+\frac{P_{v}}{P_{x}} \mathbf{I}\right]^{-1}
$$

In other words, during the first iteration of proposed iterative decision feedback receiver, MMSE compensation is used to recover the transmitted data.

Note that the symbol used in feedback could be either hard detection or soft detection. For both cases, it requires the cross correlation $R_{\hat{x} x}^{(i-1)}$ to calculate the feedforward filter $\mathbf{F}_{k}^{(i)}$ and feedback filter $\mathbf{B}_{k}^{(i)}$. Next, the parameter estimation methods will be discussed in both hard detection and soft detection. 


\section{Hard Detection}

In hard detection, the time domain feedback symbol at iteration $i$ is the hard decision output of the previous iteration, denoted as $\hat{x}_{h}^{(i-1)}(n)$. Follow the ideal in [51], the estimation of $R_{\hat{x} x}^{(i-1)}$ for hard detection is obtained as

$$
\hat{R}_{\hat{x} x}^{(i)}=\frac{1}{N} \sum_{k=1}^{N} \hat{X}_{L S}(k) \hat{X}^{(i) *}(k),
$$

where $\hat{X}_{L S}(k)$ is the Least Square (LS) estimation of the transmitted data in frequency domain obtained by $\hat{\mathbf{X}}_{k}=\mathbf{C}_{k}^{-1} \mathbf{Y}_{k}$ and a demapping operation.

\section{Soft Detection}

In the soft detection, the block wise average is replaced by the symbol wise average. The time domain estimator $\tilde{x}^{(i)}(n)$ at iteration $i$ is approximated as the sum of true symbol $x(n)$ and residential interference, i.e.,

$$
\tilde{x}^{(i)}(n)=x(n)+\epsilon,
$$

where $\epsilon$ is the residential interference with zero mean complex Gaussian distribution. Its variance is estimated as

$$
\left(\sigma_{\epsilon}^{(i)}\right)^{2}=\frac{1}{2 N} \sum_{n=1}^{N}\left|\hat{x}_{h}^{(i)}(n)-\tilde{x}^{(i)}(n)\right|^{2}
$$

Denote $\mathcal{A}$ as the set of constellation symbols. Then the a posteriori probability (APP) of detecting $\tilde{x}^{(i)}(n)=x, x \in \mathcal{A}$ can be expressed by

$$
p\left(x(n)=x \mid \tilde{x}^{(i)}(n)\right)=K e^{-\frac{\left|\tilde{x}^{(i)}(n)-x(n)\right|^{2}}{2 \sigma_{\epsilon}^{(i) 2}}},
$$

where $K$ is a normalization factor. 
The soft feedback symbol $\hat{x}_{s}^{(i)}(n)$ is given by

$$
\hat{x}_{s}^{(i)}(n)=\sum_{x \in \mathcal{A}} x \cdot p\left(x(n)=x \mid \tilde{x}^{(i)}(n)\right)
$$

For the particular case of quadrature phase shift keying (QPSK) constellation with Gray mapping, the soft feedback symbol $\hat{x}_{s}^{(i)}(n)$ in (119) can be rewritten as [53]:

$$
\hat{x}_{s}^{(i)}(n)=\frac{1}{2} \sqrt{\frac{P_{x}}{N}}\left[\tanh \left(\frac{\rho_{n}^{I}}{2}\right)+j \cdot \tanh \left(\frac{\rho_{n}^{Q}}{2}\right)\right],
$$

in which $\rho_{n}^{I}$ and $\rho_{n}^{Q}$ are the in-phase and quadrature log likelihood ratios (LLRs), given by

$$
\begin{aligned}
& \rho_{n}^{I}=\frac{\Re\left\{\tilde{x}^{(i)}(n)\right\}}{\sigma_{\epsilon}^{(i)}} \sqrt{\frac{2 P_{x}}{N}}, \\
& \rho_{n}^{I}=\frac{\Im\left\{\tilde{x}^{(i)}(n)\right\}}{\sigma_{\epsilon}^{(i)}} \sqrt{\frac{2 P_{x}}{N}} .
\end{aligned}
$$

From (120), it obtains the estimation for $R_{\hat{x} x}^{(i-1)}$ as

$$
\begin{aligned}
R_{\hat{x} x}^{(i)} & =\mathrm{E}\left[X(k) \hat{X}(k)^{(i) *}\right] \\
& =\frac{1}{2} \frac{P_{x}}{N} \sum_{n=1}^{N}\left[\tanh \left(\frac{\left|\rho_{n}^{I}\right|}{2}\right)+\tanh \left(\frac{\left|\rho_{n}^{Q}\right|}{2}\right)\right] .
\end{aligned}
$$

The soft detection based iterative IQ imbalance compensation can be extended to other constellations, where $\hat{x}_{s}^{(i)}(n)$ and $R_{\hat{x} x}^{(i)}$ can be estimated using similar approach provided in [54].

\section{Computational Complexity}

The computational complexity of the proposed receiver is evaluated in terms of the number of complex multiplications (CMUL), where one CMUL is equivalent to 4 real multiplications (RMUL). Specifically, a $N$-point DFT requires $(N / 2) \log (N)-N$ CMUL (assuming radix-2 Fast Fourier Transform (FFT) is used). For complex matrices, the 
TABLE 1: Computational Complexity Comparison of Different Receivers

\begin{tabular}{|c|c|c|c|}
\hline Procedures & MMSE & Iterative Compensation & IBDFE \\
\hline \hline Data Estimation & $N \log _{2} N-2$ & $N_{I} N \log _{2} N+2\left(N_{I} N-2 N_{I}-N+1\right)$ & $N_{I} N \log _{2} N-N$ \\
\hline FF Filter Design & $11 N-11$ & $11 N-11+(9 N-4)\left(N_{I}-1\right)$ & $2 N+(2 N+3)\left(N_{I}-1\right)$ \\
\hline FB Filter Design & N/A & $(6 N-3)\left(N_{I}-1\right)$ & $(2 N+1)\left(N_{I}-1\right)$ \\
\hline HD Parameter & N/A & $2 N\left(N_{I}-1\right)+N$ & $2 N\left(N_{I}-1\right)+N$ \\
\hline SD Parameter & N/A & $\frac{5}{2} N\left(N_{I}-1\right)$ & $\frac{5}{2} N\left(N_{I}-1\right)$ \\
\hline
\end{tabular}

inversion of a $2 \times 2$ matrix requires 6 CMUL; the multiplication of two $2 \times 2$ matrices requires 8 CMUL; and the multiplication of a $2 \times 2$ complex matrix and a $2 \times 1$ complex vector requires 4 CMUL. The data estimation in (97) requires $4(N-1)$ CMUL for each iteration, except that the first iteration requires $2(N-1)$ CMUL. The feedback filter design in (110) requires (6N-3) CMUL for each iteration. For the feedforward filter in (112), $\mathbf{C}_{k} \mathbf{C}_{k}^{H}$ is calculated once at the first iteration, so its complexity is $9 N-4$ CMUL for each iteration except that the first iteration requires $11 N-11$ CMUL. Finally, the parameter estimation for $\mathrm{HD}$ and SD requires $2 N\left(N_{I}-1\right)+N$ CMUL and $\frac{5}{2} N\left(N_{I}-1\right)$ CMUL, respectively.

Table 1 compares the complexity of the proposed iterative compensation with the MMSE compensation and conventional IBDFE receiver. It shows that the proposed receiver has higher computational complexity due to its matrix form and iterative design.

\section{Joint IQ Imbalance and Channel Estimation}

This section discusses the channel estimation of SC-FDE under IQ imbalance. The IQ imbalance and multipath channel can be jointly estimated through training sequences. For each channel estimation, it requires two training sequences, denoted as $\mathbf{x}_{1}$ and $\mathbf{x}_{2}$. The 
channel estimation can be conducted in either frequency domain or time domain. In both case, the training blocks are also guarded with CP.

\section{Frequency Domain Channel Estimation}

Let $X_{1}(k)$ and $X_{2}(k)$ be the first and second training sequence in frequency domain. $Y_{1}(k)$ and $Y_{2}(k)$ are the received frequency domain data during the two training blocks. Then the channel input-output relation in frequency domain is given by

$$
\overline{\mathbf{Y}}_{k}=\mathbf{C}_{k} \overline{\mathbf{X}}_{k}+\overline{\mathbf{V}}_{k}, \quad 1 \leq k \leq \frac{N}{2}+1
$$

where $\overline{\mathbf{Y}}_{k}$ and $\overline{\mathbf{X}}_{k}$ are constructed depending on the index $k$ :

- For $2 \leq k \leq \frac{N}{2}$,

$$
\begin{gathered}
\overline{\mathbf{Y}}_{k}=\left[\begin{array}{cc}
Y_{1}(k) & Y_{2}(k) \\
Y_{1}(N-k+2)^{*} & Y_{2}(N-k+2)^{*}
\end{array}\right], \\
\overline{\mathbf{X}}_{k}=\left[\begin{array}{cc}
X_{1}(k) & X_{2}(k) \\
X_{1}(N-k+2)^{*} & X_{2}(N-k+2)^{*}
\end{array}\right] .
\end{gathered}
$$

- For $k=1$ or $k=\frac{N}{2}+1$,

$$
\begin{gathered}
\overline{\mathbf{Y}}_{k}=\left[\begin{array}{cc}
\Re\left\{Y_{1}(k)\right\} & \Re\left\{Y_{2}(k)\right\} \\
\Im\left\{Y_{1}(k)\right\} & \Im\left\{Y_{2}(k)\right\}
\end{array}\right], \\
\overline{\mathbf{X}}_{k}=\left[\begin{array}{ll}
\Re\left\{X_{1}(k)\right\} & \Re\left\{X_{2}(k)\right\} \\
\Im\left\{X_{1}(k)\right\} & \Im\left\{X_{2}(k)\right\}
\end{array}\right] .
\end{gathered}
$$

Then the frequency domain channel estimation using MMSE criterion is given as

$$
\hat{\mathbf{C}}_{k}=\overline{\mathbf{Y}}_{k}\left(\overline{\mathbf{X}}_{k}^{H} \overline{\mathbf{X}}_{k}+\frac{P_{v}}{P_{x}} \mathbf{I}\right)^{-1} \overline{\mathbf{X}}_{k}^{H}
$$


The frequency domain estimator can be improved by transforming it into time domain and removing the noise beyond the channel length. Then it is retransformed into frequency domain for IQ imbalance compensation.

\section{Time Domain Channel Estimation}

For time domain channel estimation, let the first training sequence be real and the second one be its imaginary conversion, i.e., $\mathbf{x}_{2}=j \cdot \mathbf{x}_{1}$. According to (69), the received time domain signal during the two training blocks are given by

$$
\begin{gathered}
y_{1}(n)=\left(\psi_{1}(n)+\psi_{2}(n)\right) \otimes x(n)+\bar{v}_{1}(n), \\
y_{2}(n)=j \cdot\left(\psi_{1}(n)-\psi_{2}(n)\right) \otimes x(n)+\bar{v}_{2}(n) .
\end{gathered}
$$

Denote $\boldsymbol{\psi}_{1}, \boldsymbol{\psi}_{2}$ as $N \times 1$ vectors with first $L$ elements consisting of $\psi_{1}(l), \psi_{2}(l)(1 \leq$ $l \leq L)$, respectively, and the rest being zeros. Let $\mathbf{q}_{1}=\boldsymbol{\psi}_{1}+\boldsymbol{\psi}_{2}$ and $\mathbf{q}_{2}=j \cdot\left(\boldsymbol{\psi}_{1}-\boldsymbol{\psi}_{2}\right)$. Then (130) and (131) can be rewritten in matrix form as

$$
\begin{aligned}
& \mathbf{y}_{1}=\mathbf{X}_{c} \mathbf{q}_{1}+\mathbf{v}_{1}, \\
& \mathbf{y}_{2}=\mathbf{X}_{c} \mathbf{q}_{2}+\mathbf{v}_{2},
\end{aligned}
$$

where $\mathbf{X}_{c}$ is a $N \times N$ circular matrix consisting of training sequence $\mathbf{x}_{1}$. The MMSE estimation of $\mathbf{q}_{1}$ and $\mathbf{q}_{2}$ are given as

$$
\begin{aligned}
& \hat{\mathbf{q}}_{1}=\boldsymbol{X}_{c}^{H}\left(\boldsymbol{X}_{c} \boldsymbol{X}_{c}^{H}+\frac{\sigma_{v}^{2}}{\sigma_{x}^{2}} \mathbf{I}_{N}\right)^{-1} \mathbf{y}_{1}, \\
& \hat{\mathbf{q}}_{2}=\boldsymbol{X}_{c}^{H}\left(\boldsymbol{X}_{c} \boldsymbol{X}_{c}^{H}+\frac{\sigma_{v}^{2}}{\sigma_{x}^{2}} \mathbf{I}_{N}\right)^{-1} \mathbf{y}_{2} .
\end{aligned}
$$


where $\mathbf{I}_{N}$ is a $N \times N$ identity matrix. Then the channel impulse responses $\boldsymbol{\psi}_{1}$ and $\boldsymbol{\psi}_{1}$ can be estimated as

$$
\begin{aligned}
& \hat{\boldsymbol{\psi}}_{1}=\frac{\hat{\mathbf{q}}_{1}-j \cdot \hat{\mathbf{q}}_{2}}{2}, \\
& \hat{\boldsymbol{\psi}}_{2}=\frac{\hat{\mathbf{q}}_{1}+j \cdot \hat{\mathbf{q}}_{2}}{2} .
\end{aligned}
$$

One special case that avoids matrix inversion in (134) and (135) is to use zero autocorrelation sequence as training sequence, such as Golay sequence. For Golay training sequence, $\boldsymbol{X}_{c} \boldsymbol{X}_{c}^{H}=N \mathbf{I}_{N}$. Moreover it has been proved that Golay sequence can achieve the Cramer-Rao Lower Bound (CRLB) with Least Square estimation [55]. Hence, the signal-to-noise ratio (SNR) terms in (134) and (135) are excluded, then the estimation of $\mathbf{q}_{1}, \mathbf{q}_{2}$ are given as $\hat{\mathbf{q}}_{1}=\frac{1}{N} \boldsymbol{X}_{c}^{H} \mathbf{y}_{1}, \hat{\mathbf{q}}_{2}=\frac{1}{N} \boldsymbol{X}_{c}^{H} \mathbf{y}_{2}$.

\section{Impact Of IQ Imbalance On Channel Estimation}

Next, the impact of IQ imbalance on channel estimation is analyzed by deriving the MSE of the channel estimation. For the MMSE channel estimation in (55), the estimation error of $k^{\text {th }}$ term is given by:

$$
\begin{aligned}
\mathbf{e}_{k} & =\mathbf{C}_{k}-\hat{\mathbf{C}}_{k} \\
& =\mathbf{C}_{k}-\left(\mathbf{C}_{k} \overline{\mathbf{X}}_{k}+\mathbf{V}_{k}\right)\left(\overline{\mathbf{X}}_{k}^{H} \overline{\mathbf{X}}_{k}+\frac{P_{v}}{P_{x}} \mathbf{I}\right)^{-1} \overline{\mathbf{X}}_{k}^{H}
\end{aligned}
$$

In high SNR regime (i.e., $\frac{P_{v}}{P_{x}} \approx 0$ ), it becomes

$$
\begin{aligned}
\mathbf{e}_{k} & =\mathbf{C}_{k}-\left(\mathbf{C}_{k} \overline{\mathbf{X}}_{k}+\mathbf{V}_{k}\right)\left(\overline{\mathbf{X}}_{k}^{H} \overline{\mathbf{X}}_{k}\right)^{-1} \overline{\mathbf{X}}_{k}^{H} \\
& =\mathbf{V}_{k} \overline{\mathbf{X}}_{k}^{-1}
\end{aligned}
$$


Then the MSE of frequency domain channel estimation in (55) becomes

$$
\begin{aligned}
M S E & =\sum_{k=1}^{\frac{N}{2}+1} \mathrm{E}\left\{\operatorname{tr}\left[\mathbf{e}_{k}^{H} \mathbf{e}_{k}\right]\right\} \\
& =\sum_{k=1}^{\frac{N}{2}+1} \mathrm{E}\left\{\operatorname{tr}\left[\left(\mathbf{V}_{k} \overline{\mathbf{X}}_{k}^{-1}\right)^{H}\left(\mathbf{V}_{k} \overline{\mathbf{X}}_{k}^{-1}\right)\right]\right\} \\
& =\sum_{k=1}^{\frac{N}{2}+1} \mathrm{E}\left\{\operatorname{tr}\left[\overline{\mathbf{X}}_{k}^{-1 H} \mathbf{V}_{k}^{H} \mathbf{V}_{k} \overline{\mathbf{X}}_{k}^{-1}\right]\right\} \\
& =P_{v} \sum_{k=1}^{\frac{N}{2}+1} \operatorname{tr}\left[\overline{\mathbf{X}}_{k}^{-1 H} \overline{\mathbf{X}}_{k}^{-1}\right]
\end{aligned}
$$

From above equation, it can be seen that, with the same noise power, the performance of channel estimation in (55) is mainly dependent on the training sequence and the value of IQ imbalance is independent of the performance of channel estimation. Similar arguments also apply to (60) and (61) for time domain channel estimation.

\section{E Simulation}

This section evaluates the performance of the proposed methods in Section III and IV. Consider uncoded QPSK modulation for single block transmission of size $N=128$. A Rayleigh fading multipath channel with exponential delay power profile is adopted. The maximum channel delay is 16 . The channel is assumed to be time invariant during one block transmission. The $\mathrm{CP}$ length is set to be the maximum channel delay (including the delay caused by imperfect branch filters), i.e., $N_{c p}=20$. The performance under both perfect CSI and estimated channel is provided.

The amplitude mismatch $\alpha_{t}^{I}$ is defined in $\log$-scale as $\alpha=20 \log _{2} A \mathrm{~dB}$ with $A$ being the amplitude of the transmitted in-phase signal. Similarly, $\alpha_{t}^{Q}, \alpha_{r}^{I}$ and $\alpha_{r}^{Q}$ are all in log-scale. To demonstrate the robustness of the proposed receiver under different IQ 
imbalance, three different settings are adopted . Let $T$ be the sampling period of the analogto-digital convertor (ADC). In case 1, the IQ imbalance parameters are $\theta_{r}^{I}=\theta_{t}^{I}=5^{\circ}$, $\theta_{r}^{Q}=\theta_{t}^{Q}=-5^{\circ}, \alpha_{t}^{I}=\alpha_{r}^{I}=0.5 \mathrm{~dB}, \alpha_{t}^{Q}=\alpha_{r}^{Q}=0 \mathrm{~dB}, \xi_{t}^{I}(t)=\xi_{r}^{I}(t)=0.01 \delta(t)+$ $\delta(t-T)+0.01 \delta(t-2 T)$ and $\xi_{t}^{Q}(t)=\xi_{r}^{Q}(t)=0.01 \delta(t)+\delta(t-T)+0.2 \delta(t-2 T)$ [34]. In case 2, set $\theta_{r}^{I}=\theta_{t}^{I}=10^{\circ}, \theta_{r}^{Q}=\theta_{t}^{Q}=-10^{\circ}, \alpha_{t}^{I}=0.7 \mathrm{~dB}, \alpha_{r}^{I}=0.6 \mathrm{~dB}$, $\alpha_{t}^{Q}=\alpha_{r}^{Q}=0 \mathrm{~dB}, \xi_{t}^{I}(t)=\xi_{r}^{I}(t)=0.03 \delta(t)+\delta(t-T)+0.01 \delta(t-2 T)$ and $\xi_{t}^{Q}(t)=\xi_{r}^{Q}(t)=$ $0.02 \delta(t)+\delta(t-T)+0.08 \delta(t-2 T)$. Note that the aforementioned imbalance filters are the digital form (i.e., after sampling). The image rejection ratio (IRR) [56] of case 1 and case 2 (average over $10^{5}$ channel realization) is plotted in Figfure 20, which shows that case 2 has lower IRR (i.e., stronger mismatch between the two branches). In case 3, the amplitude and phase mismatch are randomly generated. Specifically, the amplitude mismatch is uniformly distributed within $-5 d B \sim 5 d B$ and the phase mismatch is uniformly distributed within $-5^{\circ} \sim 5^{\circ}$. The mismatch of two branch filters is the same with case 1 .

\section{Perfect Channel Knowledge}

With perfect channel knowledge, Figure 21 illustrates the BER performance of the proposed iterative IQ imbalance compensation receiver at the first four iterations under case 1. Both the performance of hard detection (HD) and soft detection (SD) are provided for comparison. Note that the first iteration of the proposed iterative receiver is equivalent to the MMSE IQ imbalance compensation. As it can be seen, both HD and SD based iterative receivers outperform the conventional MMSE IQ imbalance compensation. In particular, in high SNR regime, the HD and SD iterative receivers at forth iteration respectively have nearly $2 \mathrm{~dB}$ and $4 \mathrm{~dB}$ performance gain compared with the conventional 


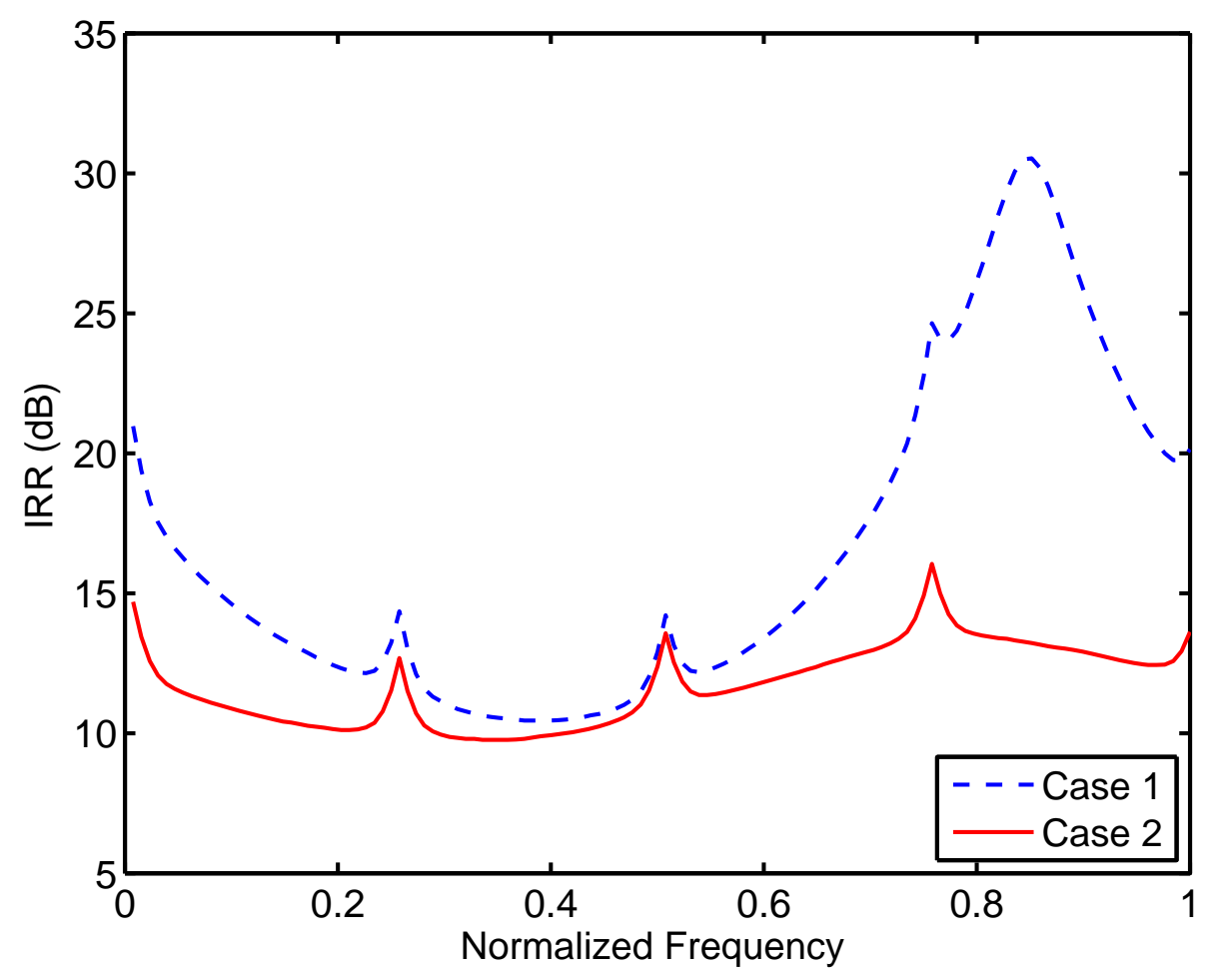

Figure 20: IRR of the case 1 and case 2.

MMSE compensation.

Figure 22 compares the BER performance of the proposed receiver and the conventional IBDFE receiver at first and forth iterations in case 1. Note that the BER curves of conventional IBDFE under ideal RF (without IQ imbalance) are also provided as benchmarks. It can be observed that, for both $\mathrm{HD}$ and SD, the conventional IBDFE without IQ imbalance compensation suffers serious performance degradation. It is worth noting that the proposed HD and SD based iterative IQ imbalance compensation receiver achieve the similar performance as the conventional IBDFE receiver with ideal RF, which means the proposed receiver can fully eliminate the effect of IQ imbalance.

Figure 23 gives the corresponding results in case 2. It shows that, without 


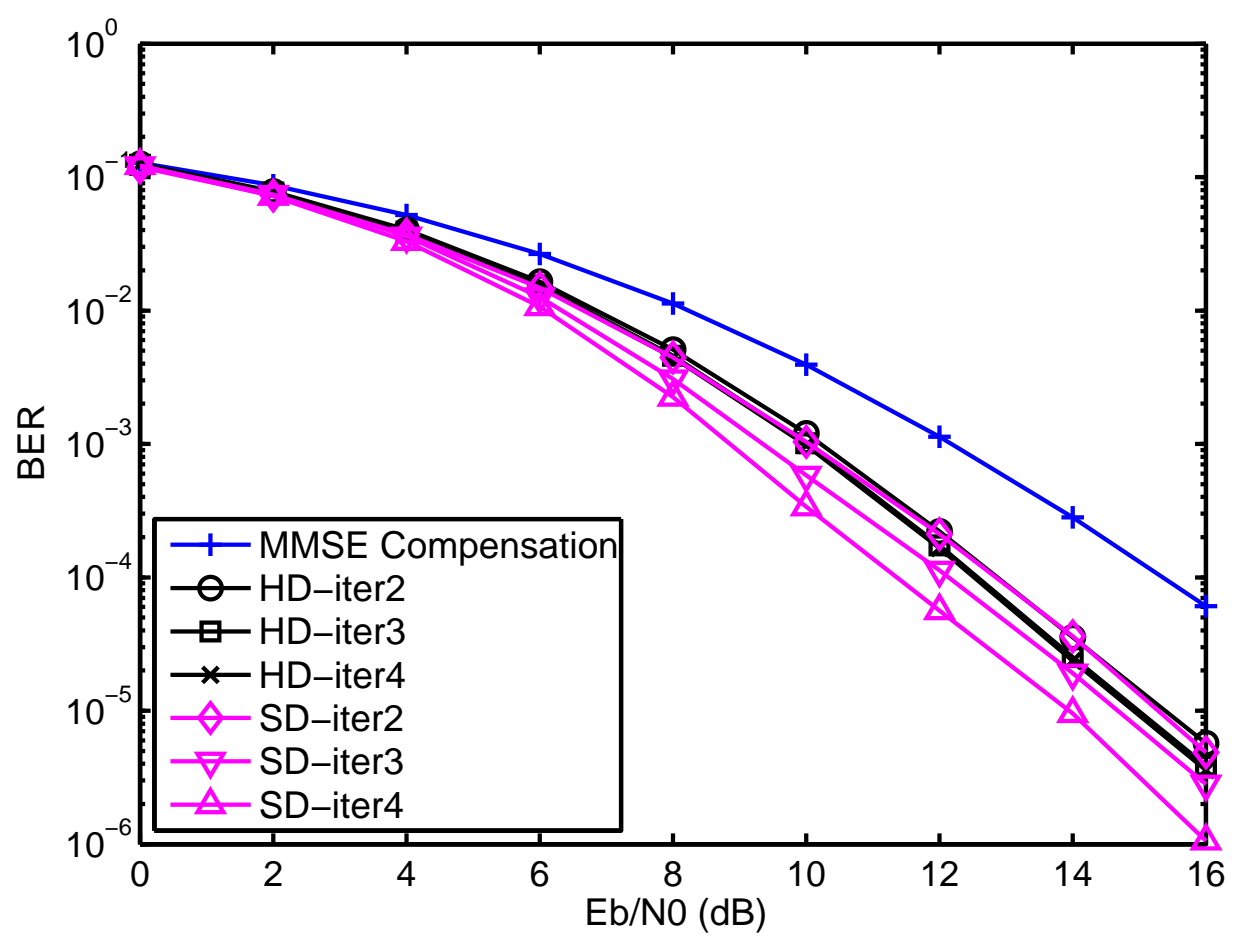

Figure 21: BERs of proposed receivers at the first four iterations with perfect CSI under case 1

compensation, the conventional IBDFE has higher performance degration compared with case 1. The BER of proposed receiver and conventional IBDFE under ideal RF also merge together. Figure 24 gives the BER curves of proposed receiver and the conventional IBDFE under random generated IQ imbalance. It shows that the proposed receiver largely outperforms the conventional IBDFE and the BER performance is close to that of ideal case without IQ imbalance.

Form these results, it is verified that the proposed receiver outperforms the conventional MMSE IQ imbalance compensation. With different values of IQ imbalance, the BERs after compensation are close to that under ideal RF. In other words, the effect of IQ imbalance can be almost fully mitigated regardless the value of IQ imbalance. Also, it 


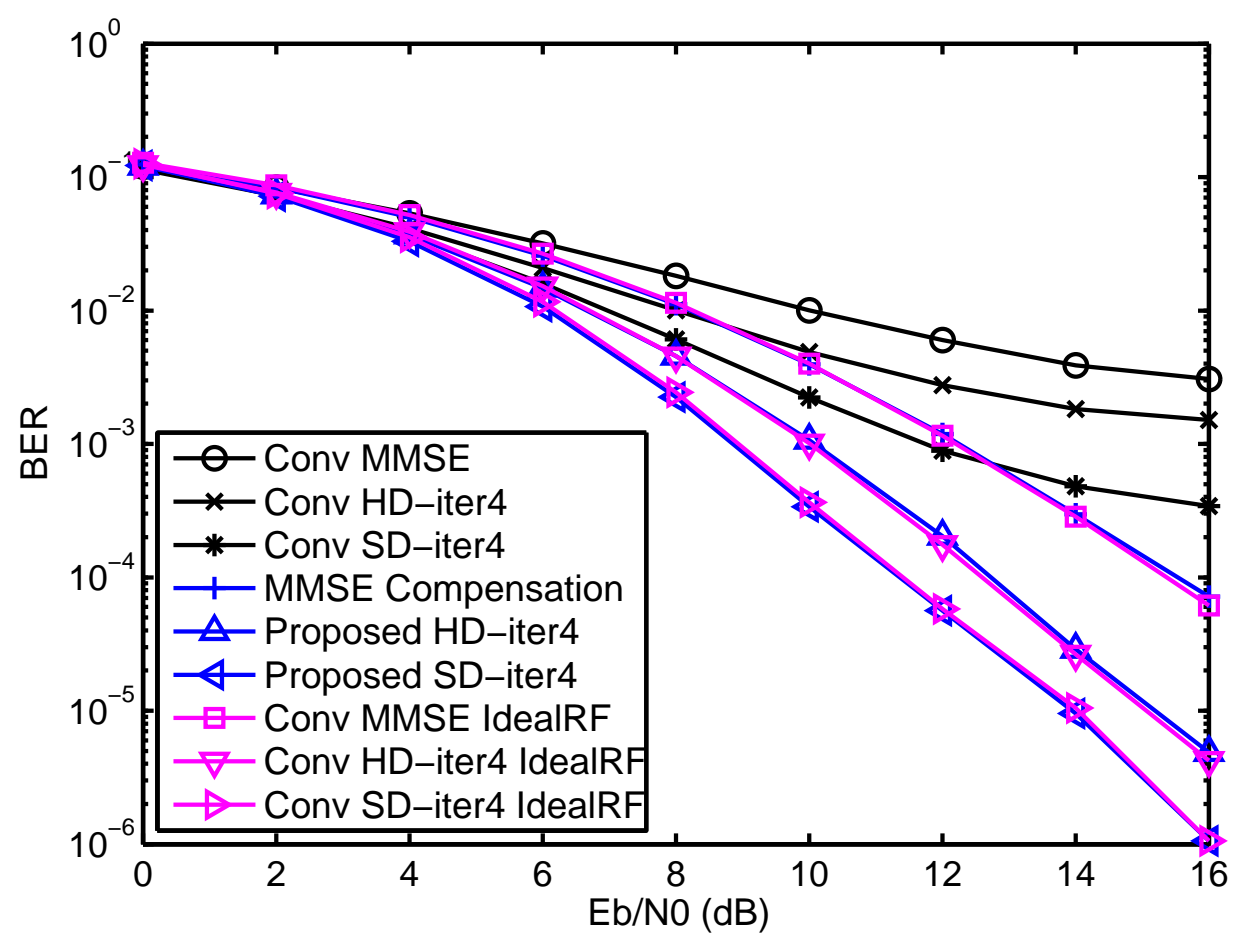

Figure 22: BER comparison of proposed receivers with conventional IBDFE receiver under case 1

is worth noting that, as show in Table 1, the complexity of proposed method is only dependent on the FFT length with given iteration times.

\section{Channel Estimation}

In the simulation of channel estimation, case 3 with randomly generated IQ imbalance parameter are adopted. For time domain channel estimation (TDCE), Golay sequence [55] of length 128 is adopted, which satisfies the training sequence to be real. For frequency domain channel estimation (FDCE), the training sequence is preferred to be flat in frequency domain, so Chu sequence [57] of length 127 is adopted. Similar with TDCE, the second training sequence in each frequency domain channel estimation is also 


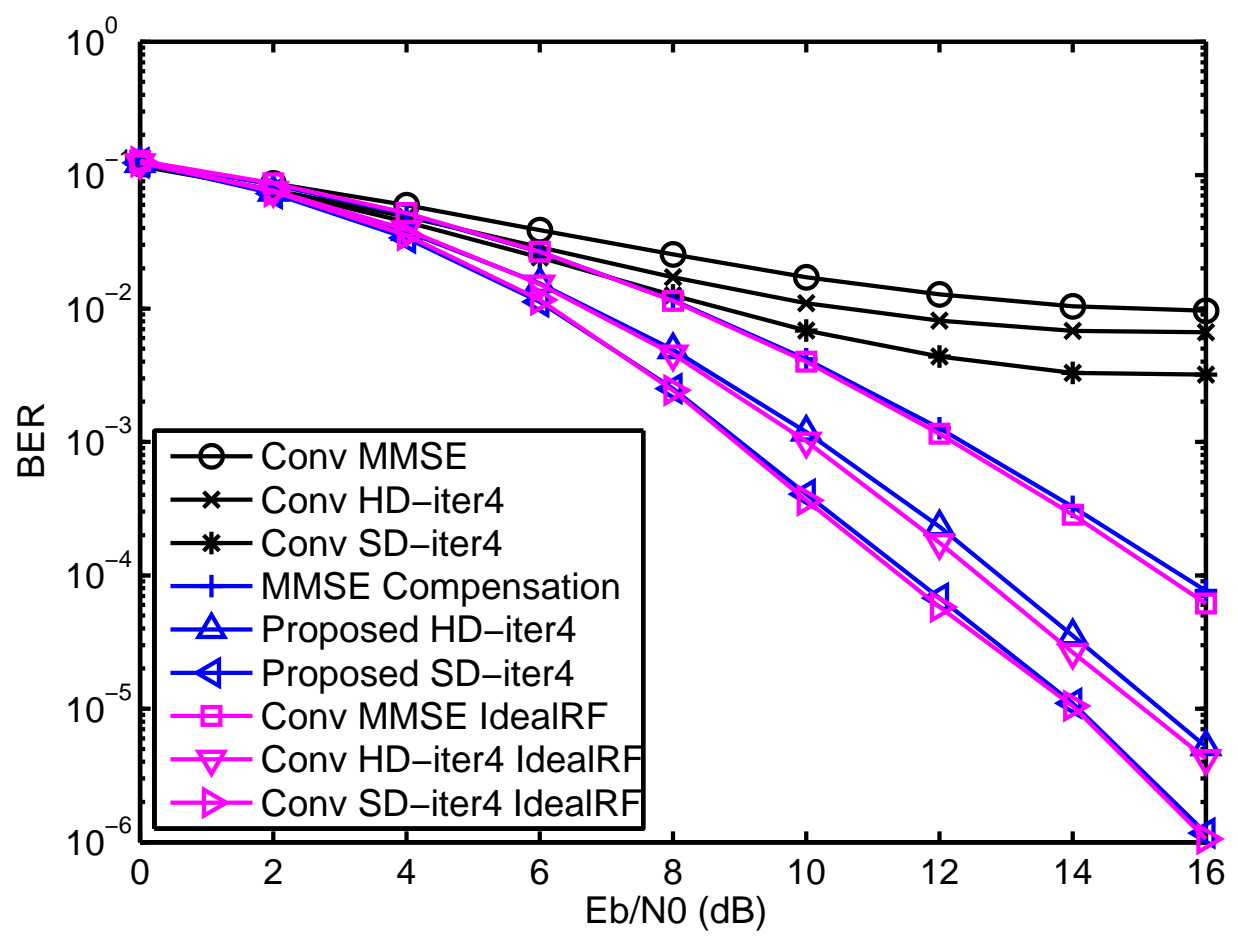

Figure 23: BER comparison of proposed receivers with conventional IBDFE receiver under case 2

set as $\mathbf{x}_{2}=j \cdot \mathbf{x}_{1}$ to guaranty the full rank of $\mathbf{C}_{k}\left(1 \leq k \leq \frac{N}{2}+1\right)$. In order to evaluate the performance of channel estimators, the normalized mean square error (MSE) of the estimator is defined as

$$
\operatorname{MSE}=\frac{|\hat{\boldsymbol{\psi}}-\boldsymbol{\psi}|^{2}}{\boldsymbol{\psi}^{H} \psi}
$$

where $\boldsymbol{\psi}$ is the concatenation of $\boldsymbol{\psi}_{1}$ and $\boldsymbol{\psi}_{2}$, i.e., $\boldsymbol{\psi}=\left[\boldsymbol{\psi}_{1}^{T} \boldsymbol{\psi}_{2}^{T}\right]^{T}$.

Figure 25 gives the normalized MSE of TDCE and FDCE under three IQ imbalance settings. Note that the MSE of FDCE with Golay sequence is also provided for comparison with TDCE with Golay sequence. The observations can be summarized as follows: (1) with given channel estimation method and training sequence, the MMSEs are nearly the same under different IQ imbalance settings, which means the impact of IQ imbalance value on 


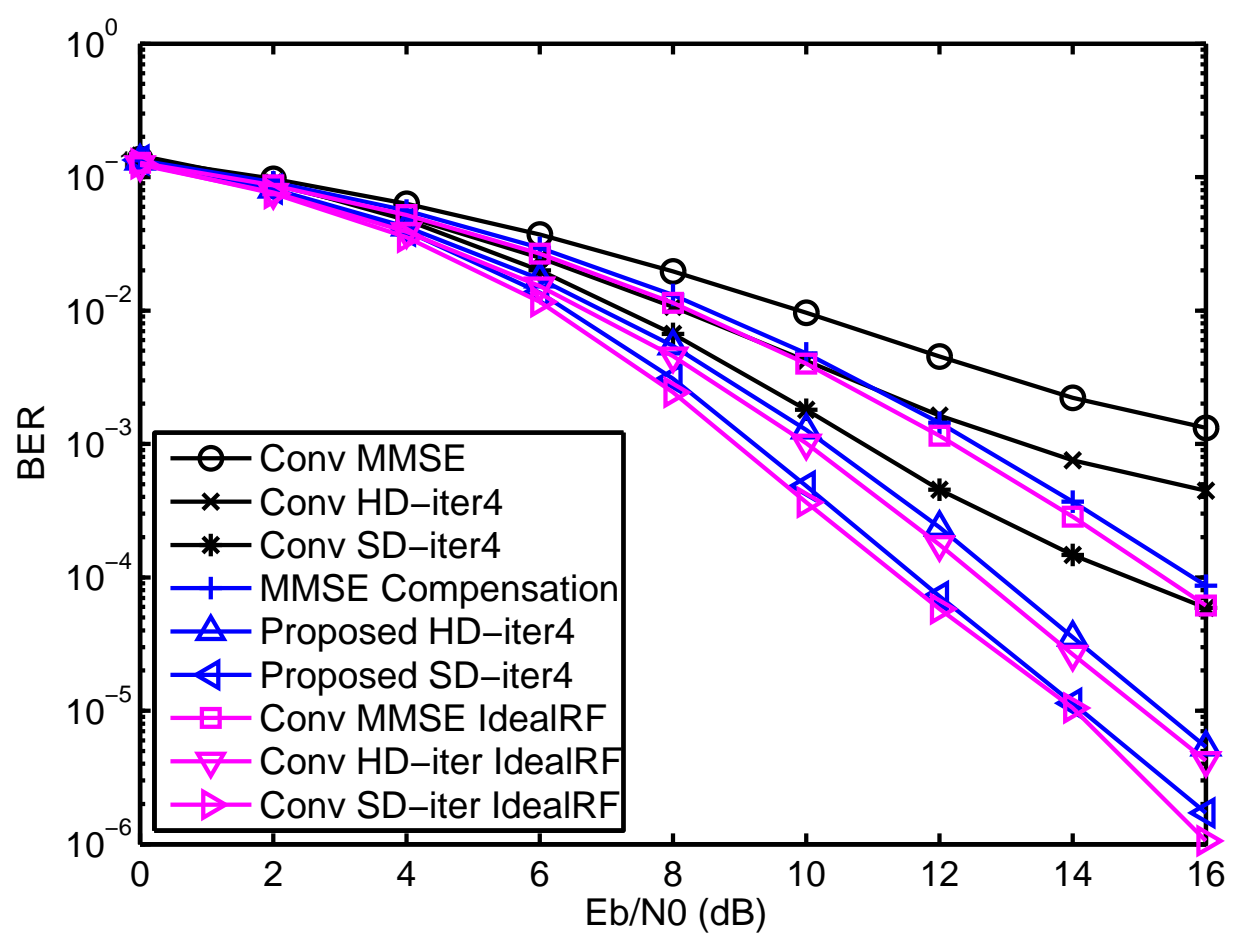

Figure 24: BER comparison of proposed receivers with conventional IBDFE receiver under case 3

the performance of channel estimation is quite limited. (2) TDCE with Golay sequence has nearly $1 \mathrm{~dB}$ performance gain compared with FDCE with Chu sequence. (3) TDCE has higher computational complexity than FDCE. However, TDCE involves two matrix multiplications with size of $N \times N$, while FDCE requires $N+2$ matrix multiplications with size of $2 \times 2$. The complexity difference between TDCE and FDCE increases with pilot length $N$ increasing. (4) FDCE Golay sequence performs worse than TDCE with Golay sequence and FDCE with Chu sequence. The reason is that the Golay sequence has strong fluctuation in frequency domain, which causes noise amplification during the matrix inversion.

Figure 26 illustrates the BER curves of the proposed iterative receivers using 


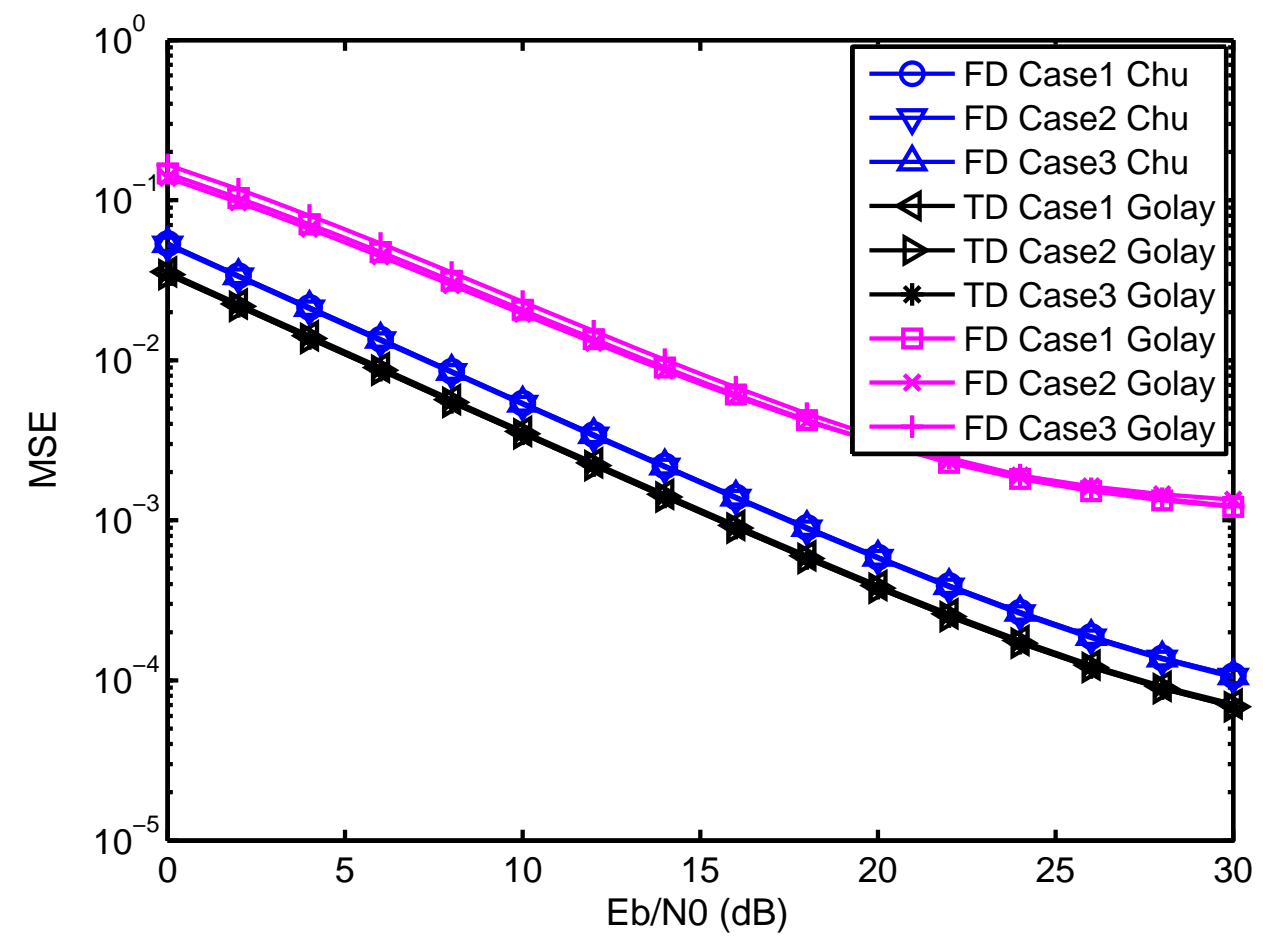

Figure 25: MSEs of frequency domain and time domain channel estimation

estimated CSI. The BER curves with perfect CSI (PSCI) are also provided as benchmark. It can be observed that, with imperfect CSI, the performance of the proposed iterative receivers significantly outperform the conventional MMSE compensation. The BER performance gap between FDCE and PSCI is about 1dB. As expected, the performance of TDCE is slightly better than that of FDCE. Finally, it is worth noting that the SD based receiver under imperfect CSI outperforms the HD based receiver with perfect CSI. 


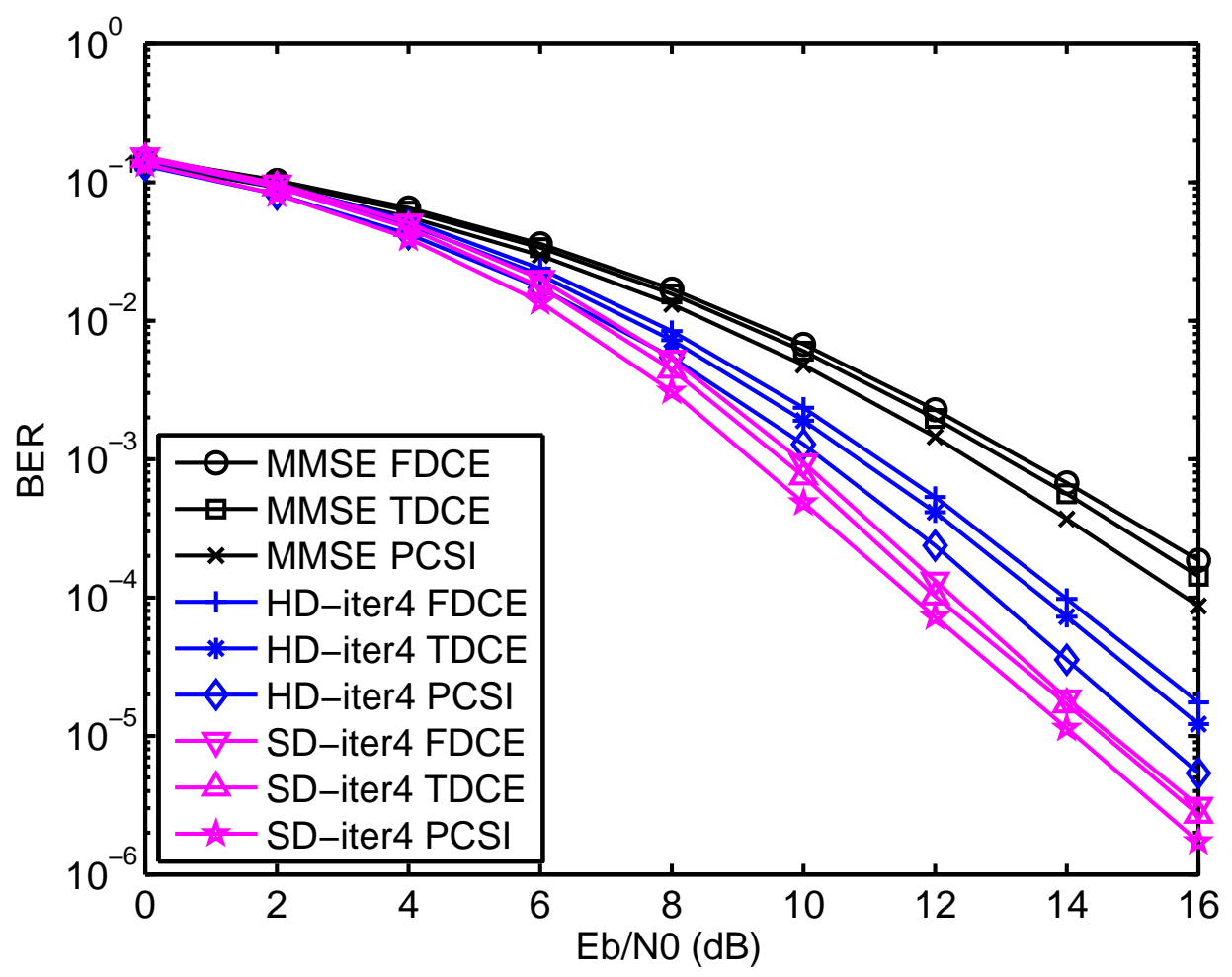

Figure 26: BERs of proposed IQ imbalance compensation receivers with estimated CSI 


\section{CHAPTER IV}

\section{ENERGY EFFICIENT TRANSMISSION STRATEGY FOR MIMO COGNITIVE RADIO}

In this chapter, the energy efficiency of multiple-input-multiple-output (MIMO) cognitive radio (CR) is investigated. It considers the CR network under overlay mode, where multiple secondary users (SUs) equipped with multiple antennas coexist with one primary user (PU). The energy efficiency optimization problems under such network model are formulated and corresponding solutions are proposed to solve those problems. Specifically, in order to maximize the energy efficiency (EE) of the CR network under SUs' transmitting power and interference constraints, multiple CR nodes control their antenna radiation patterns and allocate power among different data streams by appropriately adjusting their beamforming matrices. Three joint power allocation and transmit beamforming algorithms are introduced: network EE optimization, sum of $\mathrm{EE}$ optimization and distributed EE optimization. Since all the three problems belong to non-convex optimization, the convex relaxation is used to transform them into parametric problems for tractability. Simulation results show that the proposed algorithms significantly increase the EE compared with existing solutions. 


\section{A Background and Motivation}

$\mathrm{CR}$ is a promising approach to enhance the spectrum utilization efficiency, in which SUs are allowed to opportunistically access the licensed spectrum [58, 59] conditioning on the interference from SUs to the PU is under certain level. Meanwhile, with the multiple antenna technology, spectrum sharing becomes more efficient as beamforming has shown its potential in interference suppression [60]. In particular, CR nodes equipped with multiple antennas can adjust its radiation pattern to manage the network interference in a multi-user setting [61]. This type of CR system is usually modeled as the MIMO-CR interference channels, which is the major concern of this paper.

On the other hand, increasing energy demand and soaring energy related costs call for new designs of energy efficient communication networks. Particularly, with the popularity of mobile devices (i.e., smart phones and tablets), battery life has become a bottleneck for power hungry multimedia applications. With the explosive growth of wireless data traffic and the corresponding energy consumption, EE has become increasingly important in future communication systems. The EE of a communication network can be measured in terms of bit-per-joule capacity, which is the amount of information that can be delivered by unit energy consumed by the communication network [12]. However, for MIMO-CR interference channels, most existing studies focus on spectrum efficiency optimization [62-68], while few works can be found on EE optimization. In the literature, [69] studied the EE of MIMO-CR network under broadcast channel, which is significantly different from the MIMO-CR interference channel. In [61], the MIMO-CR interference channel was considered but the objective is to minimize the network power consumption under transmission rate constraint. Furthermore, Zhong et al. 
studied the EE optimization for the MIMO-CR interference channels using Game Theory [70]. Unfortunately, the solution provided is to simply search the best selection from a given set of transmission strategies (i.e., a codebook of precoding matrices), which does not solve the optimization problem at all. Additionally, [71] provides iterative algorithm to maximize the system EE under MIMO interference channel. However, it does not consider the CR network so that the proposed algorithm cannot protect PU's transmission. Moreover, the proposed solution is only to find a local optimum of the non-convex optimization problem (by simply letting the gradient of the objective function be zero) and does not guarantee the global optimum. Some other related studies can be found in $[72,73]$.

In this chapter, the energy-efficiency optimization of MIMO-CR network under interference channels is investigated. Joint power allocation and transmit beamforming strategies are proposed to maximize the energy efficiency of the CR network, where the transmission of $\mathrm{PU}$ is protected by the introduced interference constraint. The contributions of this chapter are given as follows:

1. It proposes a new centralized algorithm to maximize the network EE, which is defined as the ratio of the CR network's overall transmission rate to its total power consumption. In particular, the network EE optimization problem is transformed into a relaxed form by fractional programming method.

2. It proposes a new centralized algorithm to maximize the sum of all CR nodes' individual $\mathrm{EE}$, which provides an unified way to measure the $\mathrm{EE}$ of the $\mathrm{CR}$ network. It is well known that sum-of-ratio optimization is challenging [74][75]. By introducing some auxiliary variables, the sum of EE optimization problem is 
transformed into a polynomial optimization problem with better properties, and further reveal their equivalence in finding the optimal solution.

3. It propose a new distributed algorithm to maximize the individual EE, where each $\mathrm{CR}$ node iteratively finds its best transmission strategy to maximize its own EE based on local channel state information (CSI).

The rest of this chapter is organized as follows. Section II describes the network model and formulates the EE optimization problems. Section III briefly analyzes the relationship between the EE and the transmission power for a single communication link. Section IV discusses the network EE optimization, followed by the sum of EE optimization and distributed EE optimization in Section V and Section VI, respectively. Numerical results are presented in Section VII to demonstrate the effectiveness of the proposed algorithms.

\section{B System Model}

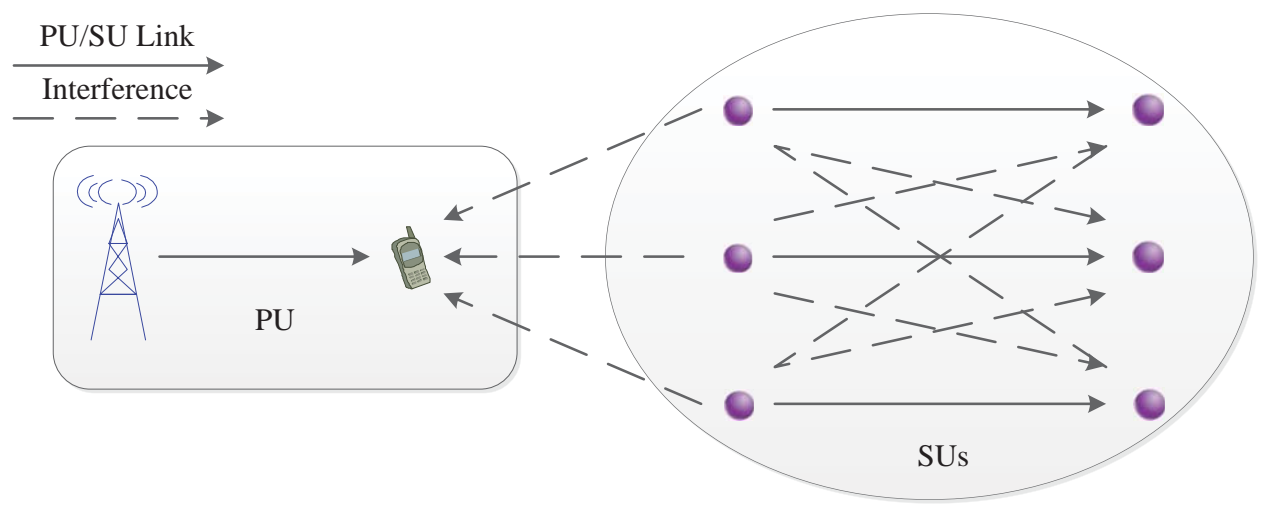

Figure 27: Network Model. 


\section{Network Description}

Consider a CR network shown in Figure 27, which consists of $I$ pairs of transmitters and receivers (i.e., I CR links) sharing the same frequency channel with the PU. Assume each CR node is equipped with $M$ antennas so that the transmitter of each CR link can send up to $M$ independent data streams. On the $i^{t h} \mathrm{CR} \operatorname{link}\left(i \in \Phi_{I}=\{1,2, \cdots, I\}\right)$, the source information is denoted as an $M \times 1$ vector $\mathbf{x}_{i}$ with $\mathbb{E}\left(\mathbf{x}_{i} \mathbf{x}_{i}^{H}\right)=\mathbf{I}$, which is multiplied by an $M \times M$ precoding matrix $\mathbf{T}_{i}$ before sending out to its desired receiver $d(i)$. The received complex baseband-equivalent signal vector $\mathbf{y}_{i}$ at the $i^{\text {th }}$ receiver can be expressed as:

$$
\mathbf{y}_{i}=\mathbf{H}_{d(i), i} \mathbf{T}_{i} \mathbf{x}_{i}+\sum_{j \in\left\{\Phi_{I} \backslash i\right\}} \mathbf{H}_{d(i), j} \mathbf{T}_{j} \mathbf{x}_{j}+\mathbf{N},
$$

where the first term in the right side of (142) is the desired signal component with $\mathbf{H}_{d(i), i}$ being the $M \times M$ channel gain matrix of the $i^{t h}$ link; the second term represents the interference from other CR transmissions with $\mathbf{H}_{d(i), j}$ being the channel gain matrix of the interference channel from CR node $j$ to $d(i) ; \mathbf{N}$ is an $M \times 1$ complex Gaussian noise vector with covariance matrix $\sigma_{N}^{2} \mathbf{I}$, representing the floor noise plus other interference (e.g., PU transmission on the same channel). For the centralized algorithms in Section IV and V, assume that there is a central controller (i.e. fusion center) having full CSI of the CR network.

The transmission rate (normalized by bandwidth) in bit/sec/Hz of the $i^{\text {th }} \mathrm{CR}$ link is given as:

$$
R_{i}=\log \operatorname{det}\left(\mathbf{I}+\mathbf{T}_{i}^{H} \mathbf{H}_{d(i), i}^{H} \mathbf{C}_{d(i)}^{-1} \mathbf{H}_{d(i), i} \mathbf{T}_{i}\right)
$$

where $\mathbf{C}_{d(i)}$ is the noise-plus-interference covariance matrix at $d(i)$, given by

$$
\mathbf{C}_{d(i)}=\sigma_{N}^{2} \mathbf{I}+\sum_{j \in\left\{\Phi_{I} \backslash i\right\}} \mathbf{H}_{d(i), j} \mathbf{T}_{j} \mathbf{T}_{j}^{H} \mathbf{H}_{d(i), j}^{H}
$$


For the $i^{\text {th }} \mathrm{CR}$ link, the total power consumption $P_{i}$ includes the transmission power and the circuit power $P_{c}$ (at both the transmitter and receiver). Apparently, $P_{i}$ should not exceed the maximum power budget $P_{\max }$, so it has:

$$
P_{i}=P_{c}+\rho \cdot \operatorname{tr}\left(\mathbf{T}_{i} \mathbf{T}_{i}^{H}\right) \leq P_{\max }
$$

where $\frac{1}{\rho} \leq 1$ representing the power amplifier efficiency.

In order to restrict the interference to the PU, SUs must be capable of preserving the performance of incumbent PU by dynamically adapting their transmission parameters to the channel conditions. Accordingly, it has the following interference constraint:

$$
\sum_{i \in \Phi_{I}} \operatorname{tr}\left(\mathbf{G}_{i} \mathbf{T}_{i} \mathbf{T}_{i}^{H} \mathbf{G}_{i}^{H}\right) \leq P_{\text {mask }}
$$

where $\mathbf{G}_{i}$ is the channel gain matrix between the $i^{\text {th }} \mathrm{SU}$ and the primary receiver (see Figure 27).

\section{Energy Efficiency Analysis}

To get some insight, the effect of transmission power on EE is analyzed under the MIMO-CR interference channels. To facilitate the discussion, denote $\mathbf{T}_{i}=\sqrt{p_{0} \eta_{i}} \tilde{\mathbf{T}}_{i}, \forall i \in$ $\Phi_{I}$, where $\tilde{\mathbf{T}}_{i}$ is the normalized beamforming matrix (i.e., $\operatorname{tr}\left(\tilde{\mathbf{T}}_{i} \tilde{\mathbf{T}}_{i}^{H}\right)=1$ ) determining the angular direction of the transmitting antennas; $p_{0}$ is the total transmission power consumed by the entire CR network; power ratio vector $\boldsymbol{\eta}=\left[\sqrt{\eta_{1}}, \sqrt{\eta_{2}}, \ldots, \sqrt{\eta_{I}}\right]$ represents the relative transmission power levels (normalized by $p_{0}$ ) among $I$ users and it satisfies $\boldsymbol{\eta} \boldsymbol{\eta}^{H}=1$. Accordingly, equation (143) can be rewritten as

$$
R_{i}=\log \operatorname{det}\left(\mathbf{I}+\mathbf{Q}_{i}^{H}\left(\frac{\sigma_{N}^{2}}{p_{0}} \mathbf{I}+\mathbf{D}_{i}\right)^{-1} \mathbf{Q}_{i}\right)
$$


where

$$
\begin{gathered}
\mathbf{Q}_{i}=\sqrt{\eta_{i}} \mathbf{H}_{d(i), i} \tilde{\mathbf{T}}_{i} \\
\mathbf{D}_{i}=\sum_{j \in\left\{\Phi_{I} \backslash i\right\}} \eta_{j} \mathbf{H}_{d(i), j} \tilde{\mathbf{T}}_{j} \tilde{\mathbf{T}}_{j}^{H} \mathbf{H}_{d(i), j}^{H}
\end{gathered}
$$
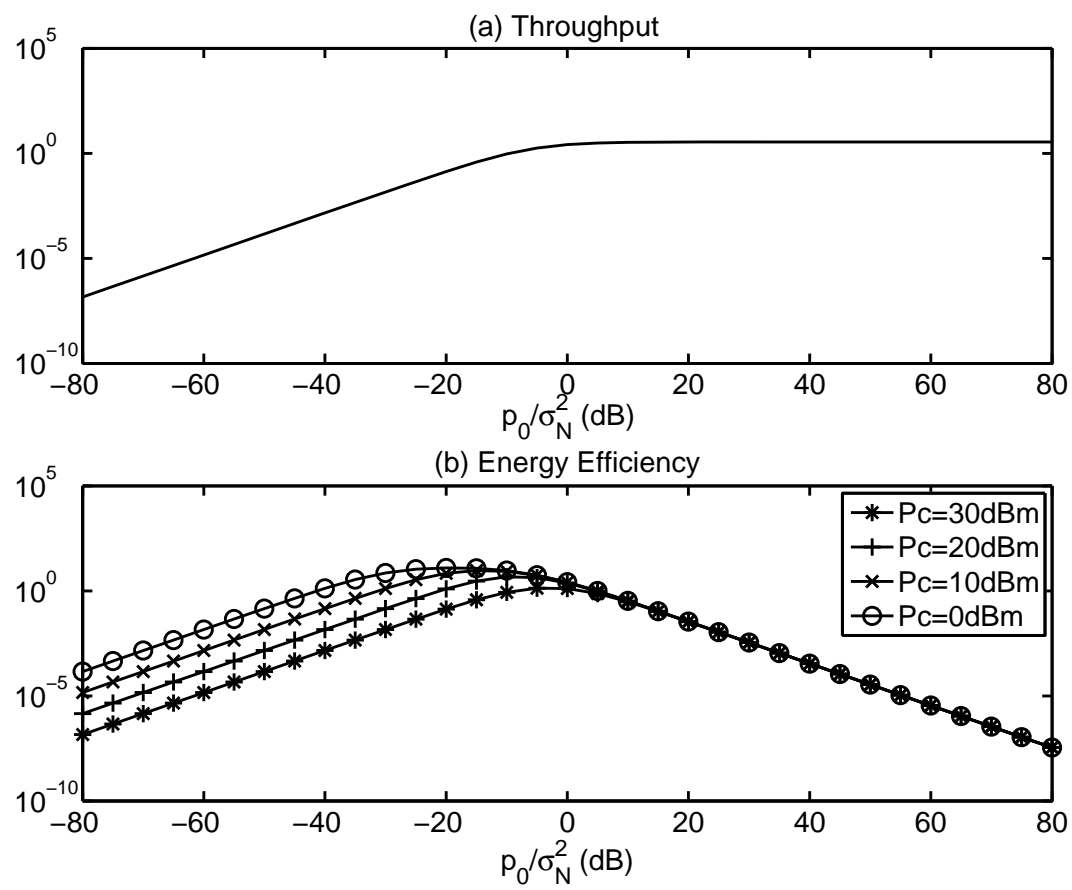

Figure 28: (a)Throughput of one link versus network total transmitting power. (b) EE of one link versus network total transmitting power.

Given precoding matrix $\tilde{\mathbf{T}}_{i}$ and power ratio vector $\boldsymbol{\eta}$, Figure 28 plots the throughput vs. EE of one CR link under different $P_{c}$ values with $\frac{p_{0}}{\sigma_{N}^{2}}$ varying from $-80 \mathrm{~dB}$ to $80 \mathrm{~dB}$. It shows that, while the throughput stays at its maximum in high power region, the EE reaches its peak value at a relatively low power region depending on $P_{c}$. This observation can be explained as follows. In high power region $\left(p_{0} \gg \sigma_{N}^{2}\right)$, it has:

$$
R_{i} \approx \log \operatorname{det}\left(\mathbf{I}+\mathbf{Q}_{i}^{H} \mathbf{D}_{i}^{-1} \mathbf{Q}_{i}\right)
$$


Since both $\mathbf{Q}_{i}$ and $\mathbf{D}_{i}$ are normalized matrices and determined by the precoding matrix $\tilde{\mathbf{T}}_{i}$ and power allocation vector $\boldsymbol{\eta}$, the transmission rate of each user is independent of $p_{0}$. As $p_{0}$ increases, the transmission rate stays the same, causing decreased EE. On the other hand, when $p_{0} / \sigma_{N}^{2}$ is small enough, the transmission rate approaches zero. Consequently, the EE of each user becomes zero due to the existence of circuit power $P_{c}$. This analysis clearly shows that, in the MIMO-CR network under interference channel, the conventional spectrum efficiency optimization may result in poor energy efficiency. Therefore, energy efficient transmission strategies are required to assure lower power consumption.

\section{Network Energy Efficiency Optimization}

This section considers the entire CR network as one entity and defines the normalized network EE [76] in bit/s/Hz/Joule as:

$$
E E_{n}=\frac{\sum_{i \in \Phi_{I}} R_{i}}{\sum_{i \in \Phi_{I}} P_{i}},
$$

which is the ratio of the network's overall throughput to its total power consumption. The objective is to maximize the network EE subject to the total power constraint (4) and interference constraint (5). Accordingly, the optimization problem can be formulated as:

$$
\begin{array}{ll} 
& \underset{\left\{\mathbf{T}_{i}, \forall i \in \Phi_{I}\right\}}{\operatorname{maximize}} \quad \frac{\sum_{i \in \Phi_{I}} R_{i}}{\sum_{i \in \Phi_{I}} P_{i}} \\
\text { s.t. } & C 1: P_{c}+\operatorname{tr}\left(\mathbf{T}_{i} \mathbf{T}_{i}^{H}\right) \leq P_{\text {max }}, \forall i \in \Phi_{I} \\
& C 2: \sum_{i \in \Phi_{I}} \operatorname{tr}\left(\mathbf{G}_{i} \mathbf{T}_{i} \mathbf{T}_{i}^{H} \mathbf{G}_{i}^{H}\right) \leq P_{\text {mask }}
\end{array}
$$

The optimization problem defined in (152) is a non-convex fractional programming problem, so finding its optimal solution can be quite challenging. Instead of solving it directly, it can be firstly transformed (152) into a relaxed form by using fractional 
programming $[72,73]$. Let $\mathbf{T}=\left[\mathbf{T}_{1}, \cdots, \mathbf{T}_{I}\right]$ be the beamforming matrix of all CR links, the following theorem is given:

Theorem 1. Given $R_{i}>0$ and $P_{i}>0$, the transmission strategy $\mathbf{T}^{*}$ for the CR network achieves the maximum network $E E \varepsilon^{*}$ if and only if $\max _{\mathbf{T}} \sum_{i=1}^{k} R_{i}(\mathbf{T})-\varepsilon^{*} \sum_{i=1}^{k} P_{i}(\mathbf{T})=$ $\sum_{i=1}^{k} R_{i}\left(\mathbf{T}^{*}\right)-\varepsilon^{*} \sum_{i=1}^{k} P_{i}\left(\mathbf{T}^{*}\right)=0$.

The detailed proof of Theorem 1 is given as follows. Let $\mathbf{T}^{*}$ be the solution of problem (152) and $S$ be the set including all eligible $\mathbf{T}$ under constraint (145) and (146). It has $\varepsilon^{*}=\frac{\sum_{i \in \Phi_{I}} R_{i}\left(\mathbf{T}^{*}\right)}{\sum_{i \in \Phi_{I}} P_{i}\left(\mathbf{T}^{*}\right)} \geq \frac{\sum_{i \in \Phi_{I}} R_{i}(\mathbf{T})}{\sum_{i \in \Phi_{I}} P_{i}(\mathbf{T})}$ for all $\mathbf{T} \in S$. Hence

$$
\begin{gathered}
\sum_{i \in \Phi_{I}} R_{i}(\mathbf{T})-\varepsilon^{*} \sum_{i \in \Phi_{I}} P_{i}(\mathbf{T}) \leq 0, \forall \mathbf{T} \in S \\
\sum_{i \in \Phi_{I}} R_{i}\left(\mathbf{T}^{*}\right)-\varepsilon^{*} \sum_{i \in \Phi_{I}} P_{i}\left(\mathbf{T}^{*}\right)=0
\end{gathered}
$$

From (153), it has $\max _{\mathbf{T}} \sum_{i \in \Phi_{I}} R_{i}(\mathbf{T})-\varepsilon^{*} \sum_{i \in \Phi_{I}} P_{i}(\mathbf{T})=0$. From (154), it can be seen that the maximum is taken on $\mathbf{T}^{*}$. Thus the first part of Theorem 1 is proved.

Let $T^{*}$ be a solution of problem $\max _{\mathbf{T}} \sum_{i=1}^{k} R_{i}(\mathbf{T})-\varepsilon^{*} \sum_{i=1}^{k} P_{i}(\mathbf{T})$ and $\sum_{i \in \Phi_{I}} R_{i}\left(\mathbf{T}^{*}\right)-\varepsilon^{*} \sum_{i \in \Phi_{I}} P_{i}\left(\mathbf{T}^{*}\right)=0$, the definition of problem above implies

$$
\begin{aligned}
& \sum_{i \in \Phi_{I}} R_{i}(\mathbf{T})-\varepsilon^{*} \sum_{i \in \Phi_{I}} P_{i}(\mathbf{T}) \\
\leq & \sum_{i \in \Phi_{I}} R_{i}\left(\mathbf{T}^{*}\right)-\varepsilon^{*} \sum_{i \in \Phi_{I}} P_{i}\left(\mathbf{T}^{*}\right)=0, \forall \mathbf{T} \in S
\end{aligned}
$$

From (155), it has $\varepsilon^{*} \geq \frac{\sum_{i \in \Phi_{I}} R_{i}(\mathbf{T})}{\sum_{i \in \Phi_{I}} P_{i}(\mathbf{T})}$ for all $\mathbf{T} \in S$. That is, $\varepsilon^{*}$ is the maximum of problem (152) and $\mathbf{T}_{i}^{*}$ is the solution of problem (152).

Theorem 1 provides a necessary and sufficient condition for the optimal solution to (152). In particular, it indicates that problem (152) can be transformed to the following 
parameterized subproblem:

$$
\begin{gathered}
\underset{\mathbf{T}}{\operatorname{maximize}} \quad \sum_{i=1}^{k} R_{i}(\mathbf{T})-\varepsilon \sum_{i=1}^{k} P_{i}(\mathbf{T}) \\
\text { s.t. } \quad c_{i, 1}=P_{c}+\operatorname{tr}\left(\mathbf{T}_{i} \mathbf{T}_{i}^{H}\right)-P_{\text {max }} \leq 0, \forall i \in \Phi_{I} \\
c_{2}=\sum_{i \in \Phi_{I}} \operatorname{tr}\left(\mathbf{G}_{i} \mathbf{T}_{i} \mathbf{T}_{i}^{H} \mathbf{G}_{i}^{H}\right)-P_{\text {mask }} \leq 0
\end{gathered}
$$

where $\varepsilon$ is the introduced positive parameter. To solve problem (152), an iterative algorithm is proposed using the Dinkelbach method [77].

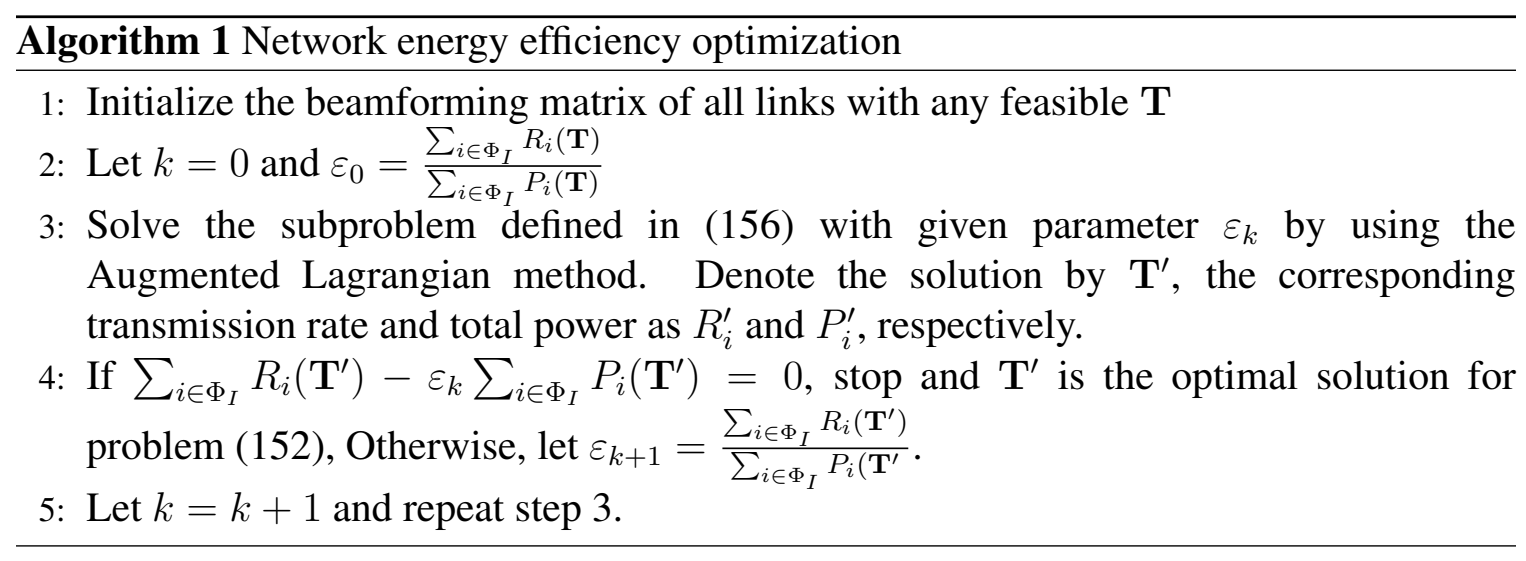

Remark 1: The convergence of the Dinkelbach method has been proved in [77]. From Algorithm 1, it can be seen that the solution to (152) can be iteratively obtained in two steps: (i) finding the solution to (156) with given $\varepsilon$; (ii) updating $\varepsilon$ until the condition in Theorem 1 is met.

Remark 2: For MIMO-CR interference channel, the transmission rate $R_{i}$ is not a convex function of T. Therefore, subproblem (156) is still non-convex due to the sumrate term in the objective function. However, the gradient method can be used to find a local optimum. Specifically, the augmented Lagrangian function for (156) is given in (157), where $\gamma_{i}\left(\forall i \in \Phi_{I}\right)$ and $\chi$ are Lagrangian multipliers associated with the total power constraint and interference constraint, and $p$ is a positive penalty parameter for violating 
the constraints. The derivative of (157) is given in (158), in which the second term can be obtained from (159); the first, third, forth and fifth term can be derived by (160), (161), (162), (163), respectively. The gradient search algorithm with Armijo step size is used to find $\mathbf{T}$ such that (158) holds for all users. The details are summarized in Algorithm 2.

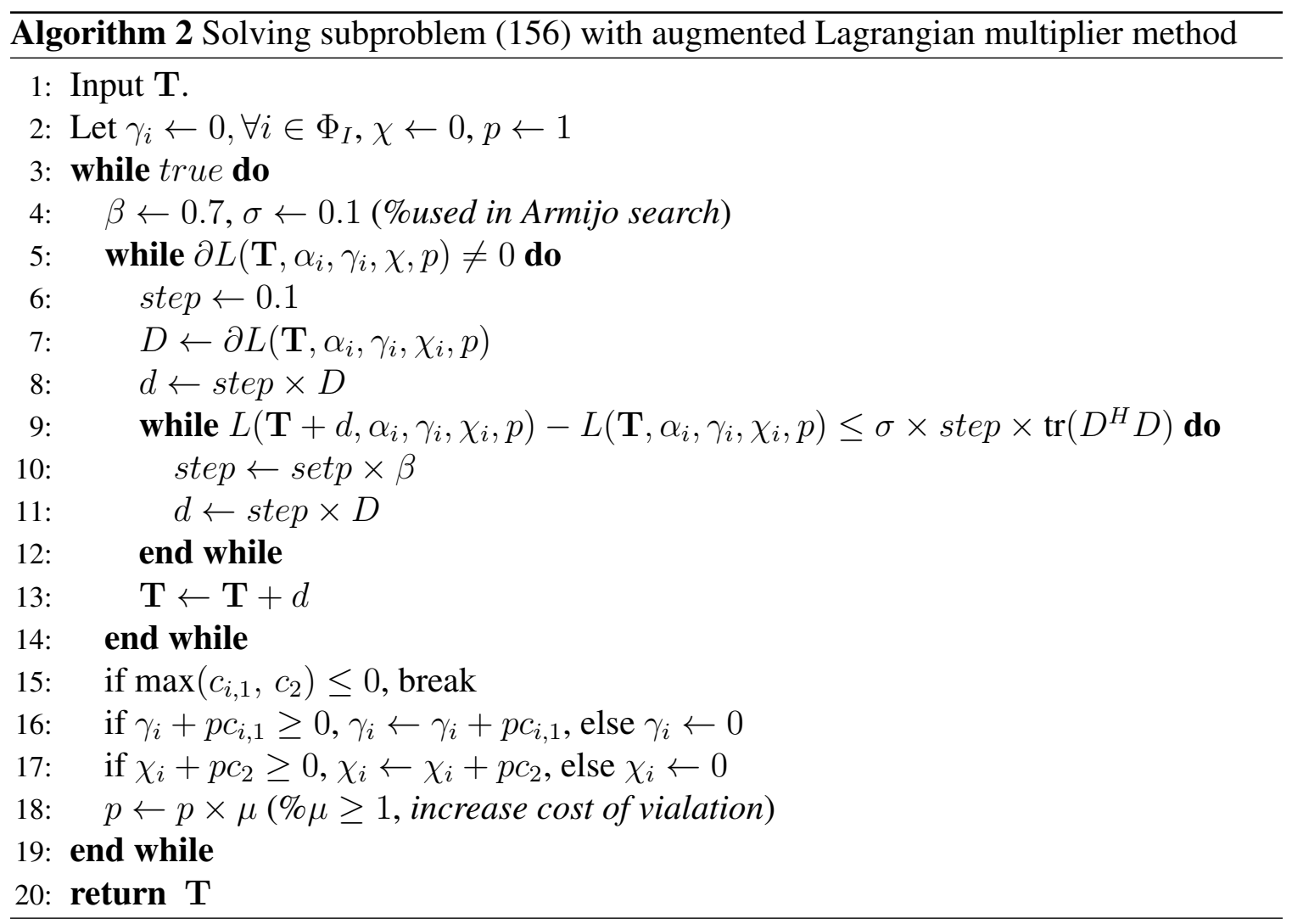

To implement Algorithm 2, it uses the following isomorphism mapping [62] from a complex matrix to a vector of real variables. The vector of variables $\mathbf{x}$ is given in (164) and the corresponding Lagrangian gradient is given in (165).

$$
\begin{aligned}
L\left(\mathbf{T}, \varepsilon, \gamma_{i}, \chi, p\right)= & \sum_{i \in \Phi_{I}} R_{i}-\varepsilon \sum_{i \in \Phi_{I}} P_{i}-\frac{p}{2} \sum_{i \in \Phi_{I}}\left\{\left(\max \left\{0, \gamma_{i}+p c_{i, 1}\right\}\right)^{2}-\gamma_{i}^{2}\right\} \\
& -\frac{p}{2}\left\{\left(\max \left\{0, \chi+p c_{2}\right\}\right)^{2}-\chi^{2}\right\}
\end{aligned}
$$




$$
\begin{aligned}
& \frac{\partial L\left(\mathbf{T}, \varepsilon, \gamma_{i}, \chi, p\right)}{\partial \mathbf{T}_{i}^{*}}=\frac{\partial R_{i}}{\partial \mathbf{T}_{i}^{*}}+\sum_{j \in \Phi_{I} \backslash i} \frac{\partial R_{j}}{\partial \mathbf{T}_{i}^{*}}-\varepsilon \frac{\partial P_{i}}{\partial \mathbf{T}_{i}^{*}}-\frac{p}{2}\left\{\frac{\partial\left\{\left(\max \left\{0, \gamma_{i}+p c_{i, 1}\right\}\right)^{2}\right\}}{\partial \mathbf{T}_{i}^{*}}\right. \\
& \left.+\frac{\partial\left\{\left(\max \left\{0, \chi+p c_{2}\right\}\right)^{2}\right\}}{\partial \mathbf{T}_{i}^{*}}\right\}=0 \\
& \frac{\partial R_{j}}{\partial \mathbf{T}_{i}^{*}}=-\mathbf{H}_{d(j), i}^{H} \mathbf{C}_{d(j), j}^{-1} \mathbf{H}_{d(j), i} \mathbf{T}_{i}+\mathbf{H}_{d(j), i}^{H}\left(\mathbf{C}_{d(j), j}+\mathbf{H}_{d(j), j} \mathbf{T}_{j} \mathbf{T}_{j}^{H} \mathbf{H}_{d(j), j}^{H}\right)^{-1} \mathbf{H}_{d(j), i} \mathbf{T}_{i} \\
& \frac{\partial R_{i}}{\partial \mathbf{T}_{i}^{*}}=\mathbf{H}_{d(i), i}^{H}\left(\mathbf{C}_{d(i)}+\mathbf{H}_{d(i), i} \mathbf{T}_{i} \mathbf{T}_{i}^{H} \mathbf{H}_{d(i), i}^{H}\right)^{-1} \mathbf{H}_{d(i), i} \mathbf{T}_{i} \\
& \frac{\partial P_{i}}{\partial \mathbf{T}_{i}^{*}}=\mathbf{T}_{i} \\
& \frac{\partial\left\{\left(\max \left\{0, \gamma_{i}+p c_{i, 1}\right\}\right)^{2}\right\}}{\partial \mathbf{T}_{i}^{*}}=\left\{\begin{array}{l}
0, \quad \text { if } \gamma_{i}+p c_{i, 1} \leq 0 \\
2 p\left(\gamma_{i}+p c_{i, 1}\right) \mathbf{T}_{i}, \text { else }
\end{array}\right. \\
& \frac{\partial\left\{\left(\max \left\{0, \chi+p c_{2}\right\}\right)^{2}\right\}}{\partial \mathbf{T}_{i}^{*}}=\left\{\begin{array}{l}
0, \quad \text { if } \chi+p c_{2} \leq 0 \\
2 p\left(\chi+p c_{2}\right) \mathbf{G}_{i}^{H} \mathbf{G}_{i} \mathbf{T}_{i}, \text { else }
\end{array}\right. \\
& \left.\mathbf{x}=\left[\Re\left[\operatorname{vec}\left(\mathbf{T}_{1}\right)\right]^{T}, \cdots, \Re\left[\operatorname{vec}\left(\mathbf{T}_{I}\right)\right]^{T}\right], \Im\left[\operatorname{vec}\left(\mathbf{T}_{1}\right)\right]^{T}, \cdots, \Im\left[\operatorname{vec}\left(\mathbf{T}_{I}\right)\right]^{T}\right]^{T} \\
& \left.\nabla_{x} L=\left[\Re\left[\operatorname{vec}\left(\frac{\partial L}{\partial \mathbf{T}_{1}^{*}}\right)\right]^{T}, \cdots, \Re\left[\operatorname{vec}\left(\frac{\partial L}{\partial \mathbf{T}_{I}^{*}}\right)\right]^{T}\right], \Im\left[\operatorname{vec}\left(\frac{\partial L}{\partial \mathbf{T}_{1}^{*}}\right)\right]^{T}, \cdots, \Im\left[\operatorname{vec}\left(\frac{\partial L}{\partial \mathbf{T}_{I}^{*}}\right)\right]^{T}\right]^{T}
\end{aligned}
$$

\section{Sum of Energy Efficiency Optimization}

The network EE characterizes the tradeoff between CR network's total income (transmission rate) and total cost (power consumption). However, it completely ignores each CR link's individual EE. In this section, it measures each CR link's individual EE and the objective is to maximize the sum of EE of all CR links under the total power and 
interference constraints. The optimization problem is formulated as:

$$
\begin{array}{ll} 
& \underset{\left\{\mathbf{T}_{i}, \forall i \in \Phi_{I}\right\}}{\operatorname{maximize}} \quad \sum_{i \in \Phi_{I}} \frac{R_{i}}{P_{i}} \\
\text { s.t. } & C 1: P_{c}+\operatorname{tr}\left(\mathbf{T}_{i} \mathbf{T}_{i}^{H}\right) \leq P_{\text {max }}, \forall i \in \Phi_{I} \\
& C 2: \sum_{i \in \Phi_{I}} \operatorname{tr}\left(\mathbf{G}_{i} \mathbf{T}_{i} \mathbf{T}_{i}^{H} \mathbf{G}_{i}^{H}\right) \leq P_{\text {mask }}
\end{array}
$$

\section{Problem Transformation}

The objective function in (166) has a sum-of-ratio form and directly finding the optimal solution is NP-complete [78]. However, motivated by [79], it can be transformed into the following equivalent parametric problem:

$$
\begin{array}{ll} 
& \underset{\left\{\mathbf{T}_{i}, \forall i \in \Phi_{I}\right\}}{\operatorname{maximize}} \sum_{i \in \Phi_{I}} \varepsilon_{i} \\
\text { s.t. } & \frac{R_{i}}{P_{i}} \geq \varepsilon_{i}, \forall i \in \Phi_{I} \\
& P_{c}+\operatorname{tr}\left(\mathbf{T}_{i} \mathbf{T}_{i}^{H}\right) \leq P_{\text {max }}, \forall i \in \Phi_{I} \\
& \sum_{i \in \Phi_{I}} \operatorname{tr}\left(\mathbf{G}_{i} \mathbf{T}_{i} \mathbf{T}_{i}^{H} \mathbf{G}_{i}^{H}\right) \leq P_{\text {mask }}
\end{array}
$$

with auxiliary variables $\varepsilon_{i}, \forall i \in \Phi_{I}$.

In order to solve (167), construct the Lagrange function in (168), where $\eta, \mu_{i}$, and $\nu_{i}, \forall i \in \Phi_{I}$ are nonnegative Lagrange multipliers. Applying the Karush-Kuhn-Tucker (KKT) condition, it obtains equation (169)-(175) given as follows.

$$
\begin{aligned}
L(\mathbf{T}, \boldsymbol{\varepsilon}, \boldsymbol{\mu}, \boldsymbol{\nu}, \eta)= & \sum_{i \in \Phi_{I}} \varepsilon_{i}-\sum_{i \in \Phi_{I}} \mu_{i}\left(\varepsilon_{i} P_{i}-R_{i}\right)-\sum_{i \in \Phi_{I}} \nu_{i}\left(P_{c}+\operatorname{tr}\left(\mathbf{T}_{i} \mathbf{T}_{i}^{H}\right)-P_{\text {max }}\right) \\
& \left.-\eta\left(\sum_{i \in \Phi_{I}} \operatorname{tr}\left(\mathbf{G}_{i} \mathbf{T}_{i} \mathbf{T}_{i}^{H} \mathbf{G}_{i}^{H}\right)\right)-P_{\text {mask }}\right)
\end{aligned}
$$




$$
\begin{gathered}
\frac{\partial L}{\partial \mathbf{T}_{i}^{*}}=\sum_{j \in\left\{\Phi_{I}\right\}} \mu_{j} \frac{\partial R_{j}}{\partial \mathbf{T}_{i}^{*}}-\mu_{i} \varepsilon_{i} \frac{\partial P_{i}}{\partial \mathbf{T}_{i}^{*}}-\frac{\partial\left\{\sum_{i \in \Phi_{I}} \nu_{i}\left(P_{c}+\operatorname{tr}\left(\mathbf{T}_{i} \mathbf{T}_{i}^{H}\right)-P_{\text {max }}\right)\right\}}{\partial \mathbf{T}_{i}^{*}} \\
-\frac{\left.\partial\left\{\eta\left(\sum_{i \in \Phi_{I}} \operatorname{tr}\left(\mathbf{G}_{i} \mathbf{T}_{i} \mathbf{T}_{i}^{H} \mathbf{G}_{i}^{H}\right)\right)-P_{\text {mask }}\right)\right\}}{\partial \mathbf{T}_{i}^{*}}=0 \\
\frac{\partial L}{\partial \varepsilon_{i}}=1-\mu_{i} P_{i}=0, \forall i \in \Phi_{I} \\
\mu_{i} \frac{\partial L}{\partial \mu_{i}}=\mu_{i}\left(R_{i}-\varepsilon_{i} P_{i}\right)=0, \forall i \in \Phi_{I} \\
\nu_{i} \frac{\partial L}{\partial \nu_{i}}=\nu_{i}\left(P_{c}+\operatorname{tr}\left(\mathbf{T}_{i} \mathbf{T}_{i}^{H}\right)-P_{\text {max }}\right)=0, \forall i \in \Phi_{I} \\
\left.\eta \frac{\partial L}{\partial \eta}=\eta\left(\sum_{i \in \Phi_{I}} \operatorname{tr}\left(\mathbf{G}_{i} \mathbf{T}_{i} \mathbf{T}_{i}^{H} \mathbf{G}_{i}^{H}\right)\right)-P_{\text {mask }}\right)=0 \\
P_{c}+\operatorname{tr}\left(\mathbf{T}_{i} \mathbf{T}_{i}^{H}\right)-P_{\text {max }} \leq 0, \forall i \in \Phi_{I} \\
\left.\sum_{i \in \Phi_{I}} \operatorname{tr}\left(\mathbf{G}_{i} \mathbf{T}_{i} \mathbf{T}_{i}^{H} \mathbf{G}_{i}^{H}\right)\right)-P_{\text {mask }} \leq 0, \forall i \in \Phi_{I}
\end{gathered}
$$

Since $\mu_{i}>0\left(\forall i \in \Phi_{I}\right),(171)$ can be rewritten as

$$
R_{i}-\varepsilon_{i} P_{i}=0, \forall i \in \Phi_{I}
$$

Theorem 2. $\mathbf{T} \in \mathbb{C}^{M \times M I}$ is an optimal solution to (167), if and only if $\mathbf{T}$ is an optimal solution to the following problem:

$$
\begin{array}{ll} 
& \underset{\left\{\mathbf{T}_{i}, \forall i \in \Phi_{I}\right\}}{\operatorname{maximize}} \sum_{i \in \Phi_{I}} \mu_{i}\left(R_{i}-\varepsilon_{i} P_{i}\right) \\
\text { s.t. } & P_{c}+\operatorname{tr}\left(\mathbf{T}_{i} \mathbf{T}_{i}^{H}\right) \leq P_{\text {max }}, \forall i \in \Phi_{I} \\
& \sum_{i \in \Phi_{I}} \operatorname{tr}\left(\mathbf{G}_{i} \mathbf{T}_{i} \mathbf{T}_{i}^{H} \mathbf{G}_{i}^{H}\right) \leq P_{\text {mask }}
\end{array}
$$

with $\varepsilon_{i}=\frac{R_{i}(\mathbf{T})}{P_{i}(\mathbf{T})}$ and $\mu_{i}=\frac{1}{P_{i}(\mathbf{T})}$.

The proof of the sufficient condition in Theorem 2 is given as follows. For fixed $\varepsilon_{i}\left(\mu_{i}, i=1, \cdots, I\right)$, it can be seen that (169), (172), (173), (174), and (175) are exactly 
the KKT conditions of problem (177). If $\mathbf{T}$ is an optimal solution to problem (177) with parameter $\varepsilon_{i}=\frac{R_{i}(\mathbf{T})}{P_{i}(\mathbf{T})}$ and $\mu_{i}=\frac{1}{P_{i}(\mathbf{T})}$, Equations (169), (172), (173), (174) and (175) must hold. Since $\mu_{i}=\frac{1}{P_{i}(\mathbf{T})}$ and $\varepsilon_{i}=\frac{R_{i}(\mathbf{T})}{P_{i}(\mathbf{T})}$, Equation (170) and (171) also hold. Therefor, the KKT condition for problem (167) holds so that $\mathbf{T}$ is also an optimal solution to problem (167). Similarly, the necessary condition of Theorem 2 can also be proved.

From Theorem 2, it can be concluded that the optimal solution to (167) can be obtained in two steps: (1) find the optimal solution to (177) under given $\mu_{i}$ and $\varepsilon_{i}$; (2) iteratively find the optimal $\mu_{i}$ and $\varepsilon_{i}$ that satisfy the conditions defined by Equation (170) and (171).

\section{Outer Loop Iteration}

The outer loop is to iteratively find the optimal $\mu_{i}$ and $\varepsilon_{i}\left(\forall i \in \Phi_{I}\right)$, under which the solution to problem (177) also satisfies the conditions defined by (170) and (171).

Let $\boldsymbol{\tau}=\left[\varepsilon_{1}, \cdots, \varepsilon_{I}, \mu_{1}, \cdots, \mu_{I}\right]$ be the parameter vector and $\mathbf{T}(\boldsymbol{\tau})$ be the solution to (177), Define the parameter vector $\phi(\tau)$ as

$$
\boldsymbol{\phi}(\boldsymbol{\tau})=\left[\phi_{1}^{1}(\boldsymbol{\tau}), \cdots, \phi_{I}^{1}(\boldsymbol{\tau}), \phi_{1}^{2}(\boldsymbol{\tau}), \cdots, \phi_{I}^{2}(\boldsymbol{\tau})\right]
$$

in which

$$
\begin{gathered}
\phi_{i}^{1}(\boldsymbol{\tau})=R_{i}(\mathbf{T}(\boldsymbol{\tau}))-\varepsilon_{i} P_{i}(\mathbf{T}(\boldsymbol{\tau})), \quad \forall i \in \Phi_{I} \\
\phi_{i}^{2}(\boldsymbol{\tau})=1-\mu_{i} P_{i}(\mathbf{T}(\boldsymbol{\tau})), \quad \forall i \in \Phi_{I}
\end{gathered}
$$

Accordingly, problem (166) is equivalent to find the optimal solution to problem (177) with parameter $\tau$, which is also the solution of the following equations:

$$
\phi(\tau)=0
$$


where 0 is a $2 N \times 1$ vector with all element being zero.

During the $k^{\text {th }}$ iteration, the Jacobian matrix of $\boldsymbol{\phi}(\boldsymbol{\tau})$ is $\mathbf{A}_{k}=-\left[\boldsymbol{\phi}^{\prime}\left(\boldsymbol{\tau}_{k}\right)\right]^{-1} \boldsymbol{\phi}\left(\boldsymbol{\tau}_{k}\right)$, where $\phi^{\prime}(\boldsymbol{\tau})$ is given by (182):

$$
\phi^{\prime}(\boldsymbol{\tau})=\left(\begin{array}{ccccccc}
P_{1}(\mathbf{T}(\boldsymbol{\tau})) & 0 & \cdots & 0 & 0 & \ldots & 0 \\
0 & P_{2}(\mathbf{T}(\boldsymbol{\tau})) & \ldots & 0 & 0 & \ldots & 0 \\
\vdots & \vdots & \ddots & \vdots & \vdots & \cdots & \vdots \\
0 & 0 & \cdots & P_{I}(\mathbf{T}(\boldsymbol{\tau})) & 0 & \cdots & 0 \\
0 & 0 & \cdots & 0 & P_{1}(\mathbf{T}(\boldsymbol{\tau})) & \cdots & 0 \\
\vdots & \vdots & \cdots & \vdots & \vdots & \ddots & \vdots \\
0 & 0 & 0 & 0 & 0 & \cdots & P_{I}(\mathbf{T}(\boldsymbol{\tau}))
\end{array}\right)
$$

During each iteration, vector $\tau$ is updated as follows:

$$
\boldsymbol{\tau}_{k+1}=\boldsymbol{\tau}_{k}+\lambda_{k} \mathbf{A}_{k}, \mathbf{A}_{k}=-\left[\boldsymbol{\phi}^{\prime}\left(\boldsymbol{\tau}_{k}\right)\right]^{-1} \boldsymbol{\phi}\left(\boldsymbol{\tau}_{k}\right)
$$

where $\lambda_{k}$ is the greatest $\xi^{n}$ satisfying

$$
\left\|\phi\left(\boldsymbol{\tau}_{k}+\xi^{n} \mathbf{A}_{k}\right)\right\| \leq\left(1-\sigma \xi^{n}\right)\left\|\phi\left(\boldsymbol{\tau}_{k}\right)\right\|
$$

and $n \in\{0,1,2, \cdots\}, \xi \in(0,1), \sigma \in(0,1)$.

Theorem 3. The modified Newton method defined by (183) and (184) converges to the solution of $\phi(\tau)=\mathbf{0}$ for any starting point $\boldsymbol{\tau}_{0}$.

A similar proof of Theorem 3 can be found in [79]. Based on (183), the modified component-wise Newton method can be rewritten as:

$$
\begin{aligned}
\varepsilon_{i, k+1} & =\varepsilon_{i, k}-\frac{\lambda_{k}}{P_{i}\left(\mathbf{T}\left(\boldsymbol{\tau}_{k}\right)\right)}\left[\varepsilon_{i, k} P_{i}\left(\mathbf{T}\left(\boldsymbol{\tau}_{k}\right)\right)-R_{i}\left(\mathbf{T}\left(\boldsymbol{\tau}_{k}\right)\right)\right] \\
& =\left(1-\lambda_{k}\right) \varepsilon_{i, k}+\lambda_{k} \frac{R_{i}\left(\mathbf{T}\left(\boldsymbol{\tau}_{k}\right)\right)}{P_{i}\left(\mathbf{T}\left(\boldsymbol{\tau}_{k}\right)\right)}
\end{aligned}
$$




$$
\begin{aligned}
\mu_{i, k+1} & =\mu_{i, k}-\frac{\lambda_{k}}{P_{i}\left(\mathbf{T}\left(\boldsymbol{\tau}_{k}\right)\right)}\left[\mu_{i, k} P_{i}\left(\mathbf{T}\left(\boldsymbol{\tau}_{k}\right)\right)-1\right] \\
& =\left(1-\lambda_{k}\right) \mu_{i, k}+\lambda_{k} \frac{1}{P_{i}\left(\mathbf{T}\left(\boldsymbol{\tau}_{k}\right)\right)}
\end{aligned}
$$

The above process that solves problem (166) can be summarized in Algorithm 3.

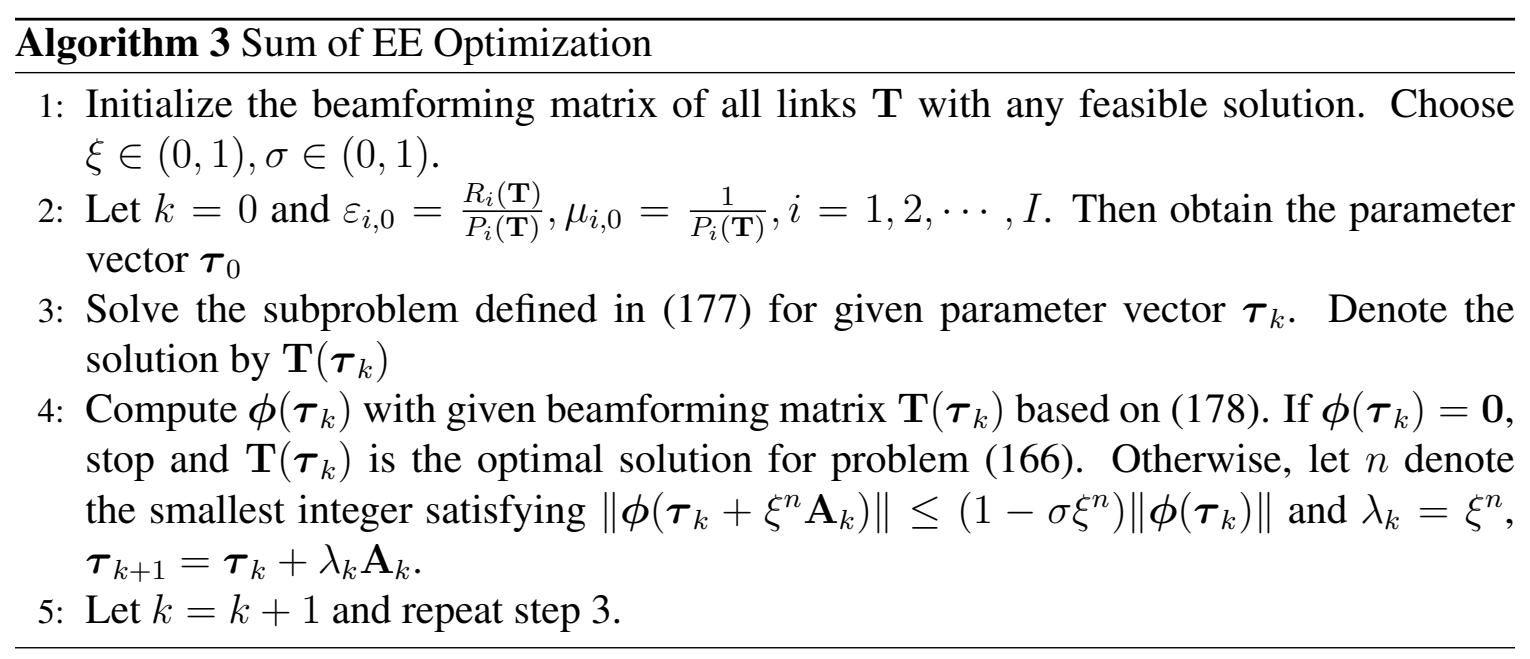

3 Solving the Subproblem

In Algorithm 3, subproblem (177) is still non-convex due to the sum of rate term in the objective function. The same step in Algorithm 2 and isomorphism mapping can be used to find a local optimum. The augmented Lagrangian function for problem (177) is given in (187), where $\gamma_{i}$ and $\chi$ are Lagrangian multipliers associated with the total power constraint and interference constraint, and $p$ is a positive penalty parameter for violating the constraints. The derivative of (187) is given in (188), in which the second term can be obtained from (159), the first, third, forth and fifth term can be derived by (160), (161), (162), (163), respectively.

$$
\begin{aligned}
L\left(\mathbf{T}, \tau, \gamma_{i}, \chi, p\right)= & \sum_{i \in \Phi_{I}} \mu_{i}\left(R_{i}-\varepsilon_{i} P_{i}\right)+\frac{p}{2} \sum_{i \in \Phi_{I}}\left\{\left(\max \left\{0, \gamma_{i}+p c_{i, 1}\right\}\right)^{2}-\gamma_{i}^{2}\right\} \\
& +\frac{p}{2}\left\{\left(\max \left\{0, \chi+p c_{2}\right\}\right)^{2}-\chi^{2}\right\}
\end{aligned}
$$




$$
\begin{aligned}
\frac{\partial L\left(\mathbf{T}, \tau, \gamma_{i}, \chi, p\right)}{\partial \mathbf{T}_{i}^{*}}= & \mu_{i} \frac{\partial R_{i}}{\partial \mathbf{T}_{i}^{*}}+\sum_{j \neq i} \mu_{j} \frac{\partial R_{j}}{\partial \mathbf{T}_{i}^{*}}-\mu_{i} \varepsilon_{i} \frac{\partial P_{i}}{\partial \mathbf{T}_{i}^{*}}+\frac{p}{2}\left\{\frac{\partial\left\{\left(\max \left\{0, \gamma_{i}+p c_{i, 1}\right\}\right)^{2}\right\}}{\partial \mathbf{T}_{i}^{*}}\right. \\
& \left.+\frac{\partial\left\{\left(\max \left\{0, \chi+p c_{2}\right\}\right)^{2}\right\}}{\partial \mathbf{T}_{i}^{*}}\right\}
\end{aligned}
$$

\section{E Distributed Energy Efficiency Optimization}

Both the centralized optimization algorithms in Section $\mathrm{C}$ and $\mathrm{D}$ require a fusion center with full knowledge of CSI and power allocation of all CR links, which causes significant information exchange overhead. On the other hand, in some practical CR networks, each node may only care its own EE. In this section, it considers a distributed EE optimization problem, where each CR link optimizes its beamforming matrix to maximize its own EE based on local CSI. The optimization problem is formulated as follows:

$$
\begin{array}{ll} 
& \underset{\left\{\mathbf{T}_{i}, \forall i \in \Phi_{I}\right\}}{\operatorname{maximize}} \quad \frac{R_{i}}{P_{i}} \\
\text { s.t. } & C 1: P_{c}+\operatorname{tr}\left(\mathbf{T}_{i} \mathbf{T}_{i}^{H}\right) \leq P_{\text {max }}, \forall i \in \Phi_{I} \\
& C 2: \sum_{i \in \Phi_{I}} \operatorname{tr}\left(\mathbf{G}_{i} \mathbf{T}_{i} \mathbf{T}_{i}^{H} \mathbf{G}_{i}^{H}\right) \leq P_{\text {mask }} .
\end{array}
$$

Assumed that $\mathbf{G}_{i}$ and $\left\{\mathbf{H}_{d(i), j} \mathbf{T}_{j}\right\}\left(\forall j \in \Phi_{I}\right)$ can be obtained at the $i^{\text {th }}$ receiver. Note that the optimization is performed at the receiver side and the transmitter $i$ obtains the optimized $\mathbf{T}_{i}$ through a feedback channel. Similar to the network EE optimization 
problem, problem (189) can also be transformed into an equivalent subproblem:

$$
\begin{array}{ll} 
& \underset{\left\{\mathbf{T}_{i}, i \in \Phi_{I}\right\}}{\operatorname{maximize}} \quad R_{i}-\alpha_{i} P_{i} \\
\text { s.t. } & c_{i, 1}=P_{c}+\operatorname{tr}\left(\mathbf{T}_{i} \mathbf{T}_{i}^{H}\right)-P_{\text {max }} \leq 0, \forall i \in \Phi_{I} \\
& c_{2}=\sum_{i \in \Phi_{I}} \operatorname{tr}\left(\mathbf{G}_{i} \mathbf{T}_{i} \mathbf{T}_{i}^{H} \mathbf{G}_{i}^{H}\right)-P_{\text {mask }} \leq 0
\end{array}
$$

where $\alpha_{i}$ is the positive parameter for the $i^{t h}$ link. Note that problem (190) is convex w.r.t. $\mathbf{T}_{i}$ and can be solved by standard convex optimization method. In particular, the augmented Lagrangian function is constructed in (191), where $p$ is a positive penalty parameter (for violating the constraints), and $\gamma_{i}$ and $\lambda_{i}$ are nonnegative Lagrangian multipliers of $i^{\text {th }}$ link. The derivative of (191) is given in (192). The distributed EE optimization is summarized in Algorithm 4.

$$
\begin{aligned}
L\left(\mathbf{T}_{i}, \alpha_{i}, \gamma_{i}, \lambda_{i}, p\right)=P_{i} & -\alpha_{i} R_{i}+\frac{p}{2}\left\{\left(\max \left\{0, \gamma_{i}+p c_{i, 1}\right\}\right)^{2}-\gamma_{i}^{2}\right\}+\frac{p}{2}\left\{\left(\max \left\{0, \lambda_{i}+p c_{2}\right\}\right)^{2}-\lambda_{i}^{2}\right\} \\
\frac{\partial L\left(\mathbf{T}_{i}, \alpha_{i}, \gamma_{i}, \lambda_{i}, p\right)}{\partial \mathbf{T}_{i}^{*}}= & \frac{p}{2}\left\{\frac{\partial\left\{\left(\max \left\{0, \gamma_{i}+p c_{i, 1}\right\}\right)^{2}\right\}}{\partial \mathbf{T}_{i}^{*}}+\frac{\partial\left\{\left(\max \left\{0, \lambda_{i}+p c_{2}\right\}\right)^{2}\right\}}{\partial \mathbf{T}_{i}^{*}}\right\} \\
& +\frac{\partial P_{i}}{\partial \mathbf{T}_{i}^{*}}-\alpha_{i} \frac{\partial R_{i}}{\partial \mathbf{T}_{i}^{*}}=0
\end{aligned}
$$

\footnotetext{
Algorithm 4 Distributed energy efficiency optimization

1: For the $i^{\text {th }}$ link, input $\mathbf{T}_{j}, \forall j \in\left\{\Phi_{I} \backslash i\right\}$.

2: Choose any feasible precoding matrix $\mathbf{T}_{i}$ and calculate $R_{i}$ and $P_{i}$, then set $\alpha_{i}=\frac{P_{i}}{R_{i}}$.

3: For a given $\alpha_{i}$, solve the subproblem in (190). Denote the solution as $\mathbf{T}_{i}^{\prime}$, the corresponding transmission rate and total power as $R_{i}^{\prime}$ and $P_{i}^{\prime}$, respectively.

4: If $P_{i}^{\prime}-\alpha_{i} R_{i}^{\prime}=0$, stop and return $\mathbf{T}_{i}^{\prime}$ as the optimal precoding matrix for the $i^{\text {th }}$ link. Otherwise, update $\alpha_{i}=\frac{P_{i}^{\prime}}{R_{i}^{\prime}}$ and go to step 3 .

5: Repeat step 1-4 among all links, i.e., $i=1,2, \cdots, I$, until convergence.
} 


\section{F Simulation Results}

In this section, numerical results are given to evaluate the convergence and effectiveness of the proposed EE optimization algorithms. The proposed algorithms are compared with existing centralized energy efficient algorithms, which are denoted as CEE1 [80] and CEE2 [71]. Note that CEE2 is designed for MIMO interference channels without power and interference constraints, so its solution may violate these constraints in the CR network. Meanwhile, to show the trade off between spectrum efficiency and energy efficiency, the two transmission strategies in [81] that maximizes the spectrum efficiency of MIMO-CR network are also given for comparison. One is known as Greedy Algorithm, where each SU acts selfishly to maximize its own transmission rate using only local CSI (the same assumption with the proposed distributed EE algorithm (DEE)); the other is the centralized sum of rate algorithm (SUMR) that maximizes the CR network's

overall throughput. For fairness, the widely used sum of EE $\sum_{i \in \Phi_{I}} \frac{R_{i}}{P_{i}}$ is adopted as the performance metric to compare the performance of different algorithms.

For the setup, consider 4 SUs in the CR network sharing the licensed spectrum with one PU. Each CR node is equipped with two antennas. The large scale fading is determined by $d^{-a}$, where $d$ is the distance between the transmitter and receiver, and a=3.5 is the freespace attenuation factor. The small scale fading is Rayleigh distributed. The maximum transmission power $P_{\max }$ is set to be $3 \mathrm{~W}$ and the interference constraint $P_{\text {mask }}=1 \mathrm{~W}$. The interference from SUs to PU is normalized by the path loss. The efficiency of power amplifier is 0.38. Note that the sum of EE (SEE) and network EE (NEE) algorithms are not guaranteed to find the global optimum, since they only find a local optimum in solving the non-convex subproblems. However, analytical results in Section B.2 can be used the 


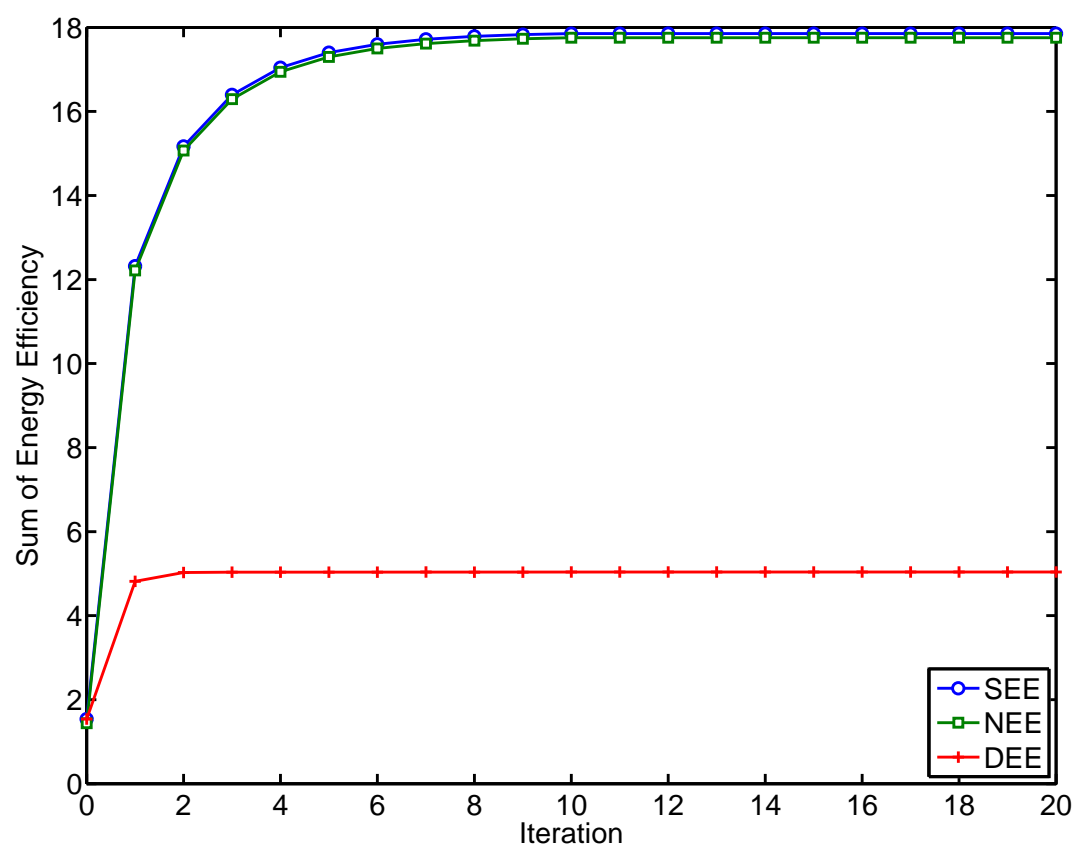

Figure 29: Convergence of the proposed Algorithms

to choose the starting point for SEE and NEE to assure a higher chance of finding the global optimum. As shown in Figure 28, power level $p_{0}$ has significant impact on the EE. Given arbitrary normalized beamforming matrix $\tilde{\mathbf{T}}_{i}^{0}\left(\forall i \in \Phi_{I}\right)$ and power ratio vector $\boldsymbol{\eta}^{0}$, the optimal $p_{0}^{*}$ can be found by linear search. Then the starting point for NEE and SEE is set as $\mathbf{T}_{i}=\sqrt{p_{0}^{*} \eta_{i}^{0}} \tilde{\mathbf{T}}_{i}^{0}, \forall i \in \Phi_{I}$, where $\boldsymbol{\eta}^{0}$ is a uniform allocation vector and $\tilde{\mathbf{T}}_{i}^{0}=\frac{1}{\sqrt{2}} \mathbf{I}$.

Firstly, consider an ideal case where the path loss of the serving/interference channels are identical (Scenario 1). In this case, it can easily quantify the interference level of the CR network. Let $A=d_{1} / d_{2}$ be the relative distance between different links, where $d_{1}$ is the distance between a transmitter and its unintended receivers (including PU), $d_{2}$ is the distance from a transmitter to its desired receiver. Obviously, $A$ naturally characterizes the interference level of the CR network (i.e., the interference level increases with $A$ ). Figure 29 shows the sum of EE versus the number of iterations for the three 


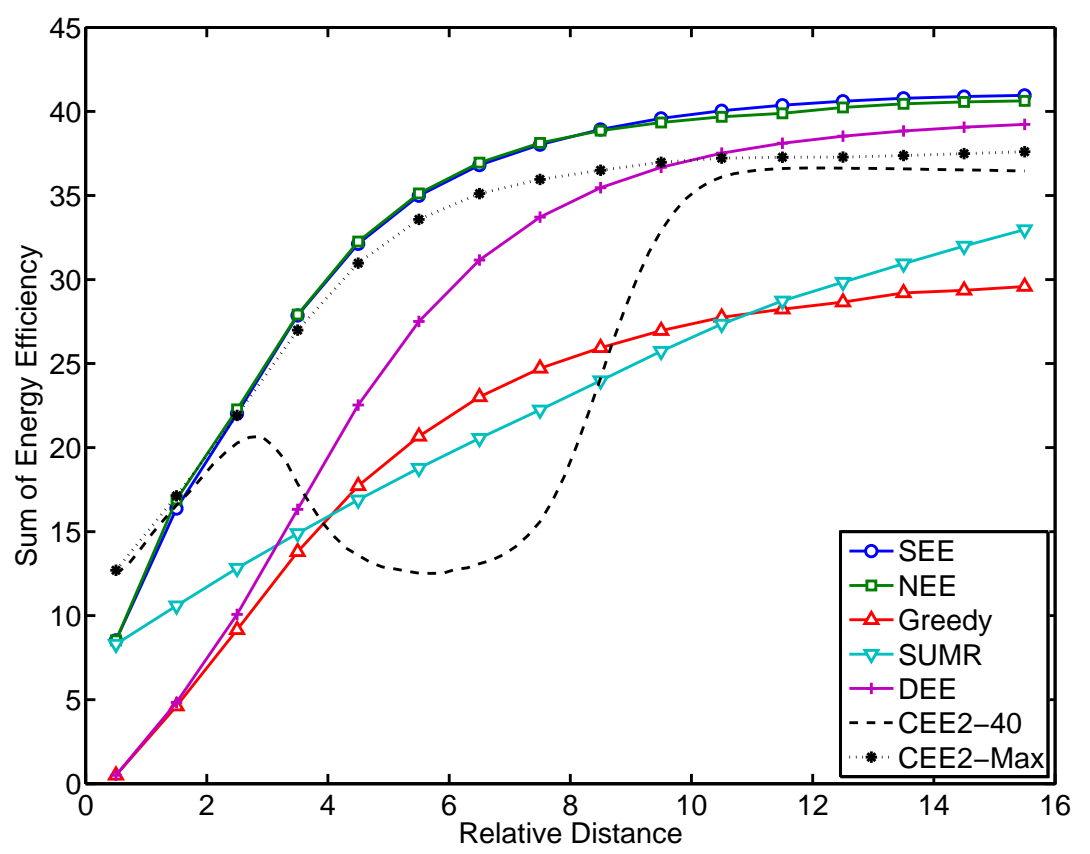

Figure 30: Sum of EE versus relative distance $A$.

proposed algorithms with circuit power $P_{c}=1 \mathrm{~W}, \sigma_{N}^{2}=1 \mathrm{~mW}$ and $A=3$. The average is taken over the channel gain matrix, whose elements are randomly generated according to i.i.d. complex Gaussian distribution with zero-mean and unit-variance. It can be observed from Figure 29 that: (1) SEE and NEE have similar performance and they converge after 10 iterations; (2) DEE converges must faster (in two iterations) but the performance gap to the centralize algorithms is huge.

In order to compare the energy and spectrum efficiencies of the proposed algorithms under different interference levels, the simulation results with relative distance $A$ changing from 0.5 to 15.5 is given. For comparison of the energy efficiency of different algorithms, Figure 30 shows the sum of EE versus the relative distance. It can be observed that: (1) the proposed NEE and SEE algorithms have similar performance and achieve the highest energy-efficiency. (2) the proposed DEE algorithm outperforms the greedy 


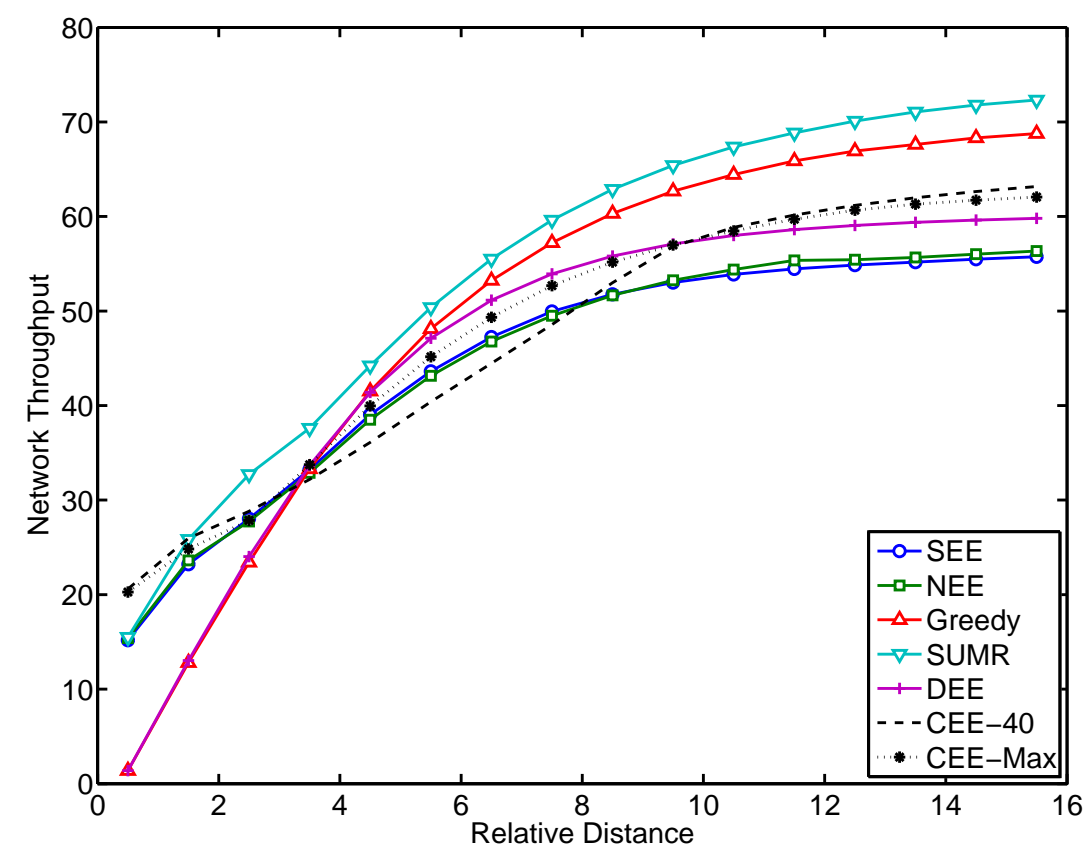

Figure 31: Network throughput versus relative distance.

algorithm and CEE2 (when $A>10$ ) and the performance gaps increase with the relative distance; (3) At high interference level, SUMR outperforms DEE, since SUMR has global CSI and can intentionally lower the transmission power to get higher EE. However, when the interference level is low, the proposed DEE achieves higher EE than SUMR. (4) At some interference levels, CEE2 may deviate from the optimum during the iteration process. Therefore, both its maximum EE during 40 iterations (CEE-Max) and its EE at the 40th iteration (CEE2-40) are given.

For spectrum efficiency comparison, Figure 31 shows the total network throughput (sum-rate) as a function of $A$. As expected, SUMR has the highest sum rate when $A>1.5$. The network throughput gap between the spectrum efficient algorithms (Greedy, SUMR) and energy efficient algorithms (SEE, NEE, DEE) increases with $A$. At low interference level (large $A$ ), SEE and NEE have the lowest transmission rate. When the interference is 


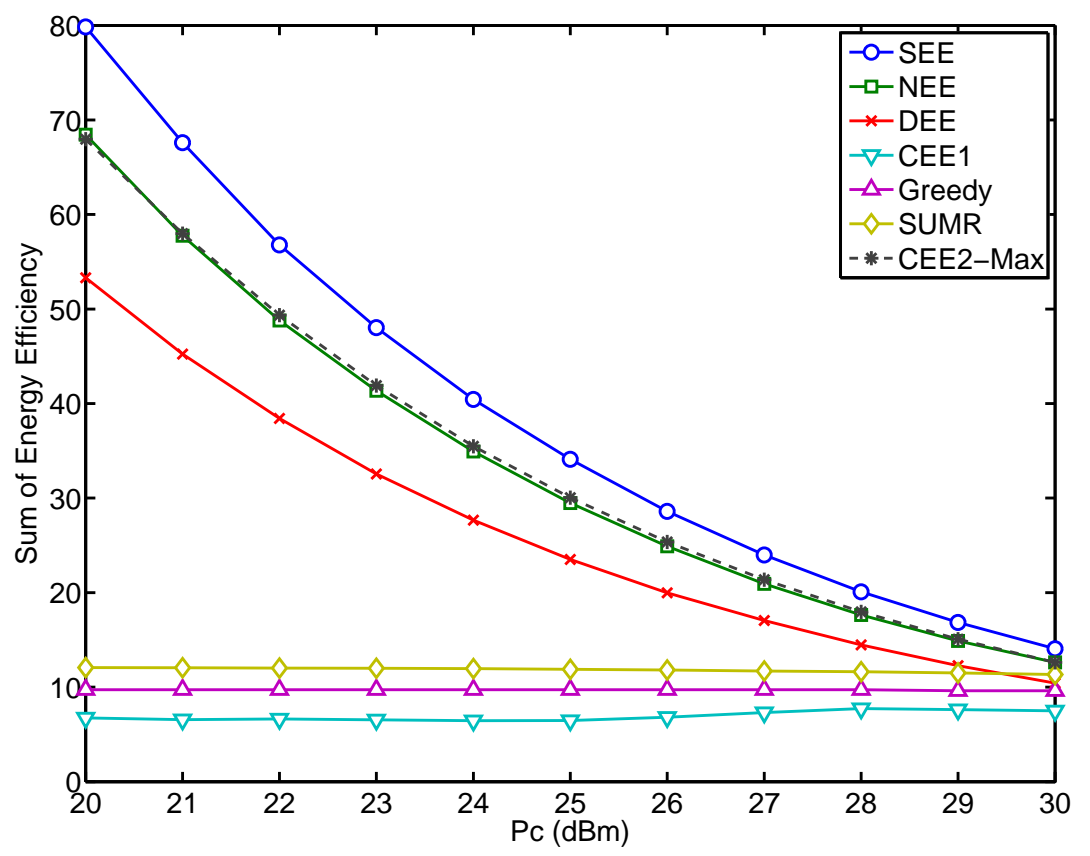

Figure 32: Sum of EE in Scenario 2.

extremely high $(0.5 \geq A<1.5)$, CEE2 offers the best performance in terms of both $\mathrm{EE}$ and spectrum efficiency.

Next, a more practical scenario (Scenario 2) is considered, where the distances between two CR nodes are random generated. Specifically, $d_{2}$ is uniformly distributed over $1-50 \mathrm{~m}$, while $d_{1}$ is uniformly distributed over $1-100 \mathrm{~m}$. The background noise $\sigma_{N}^{2}$ (including floor noise and interference from PU) is $30 \mathrm{dBm}$. Figure 32 shows the sum of EE versus circuit power for different algorithms. It shows that: (1) the proposed SEE achieves the best performance, while NEE and CEE2-Max have similar performance; (2) the performance of SEE, NEE, CEE2-Max, and DEE decrease with the circuit power, while the performance of CEE1 and greedy algorithms barely change.

Figure 33 shows the network throughput versus the circuit power. It shows that the network throughput of the proposed algorithms and CEE2-Max increases with the circuit 


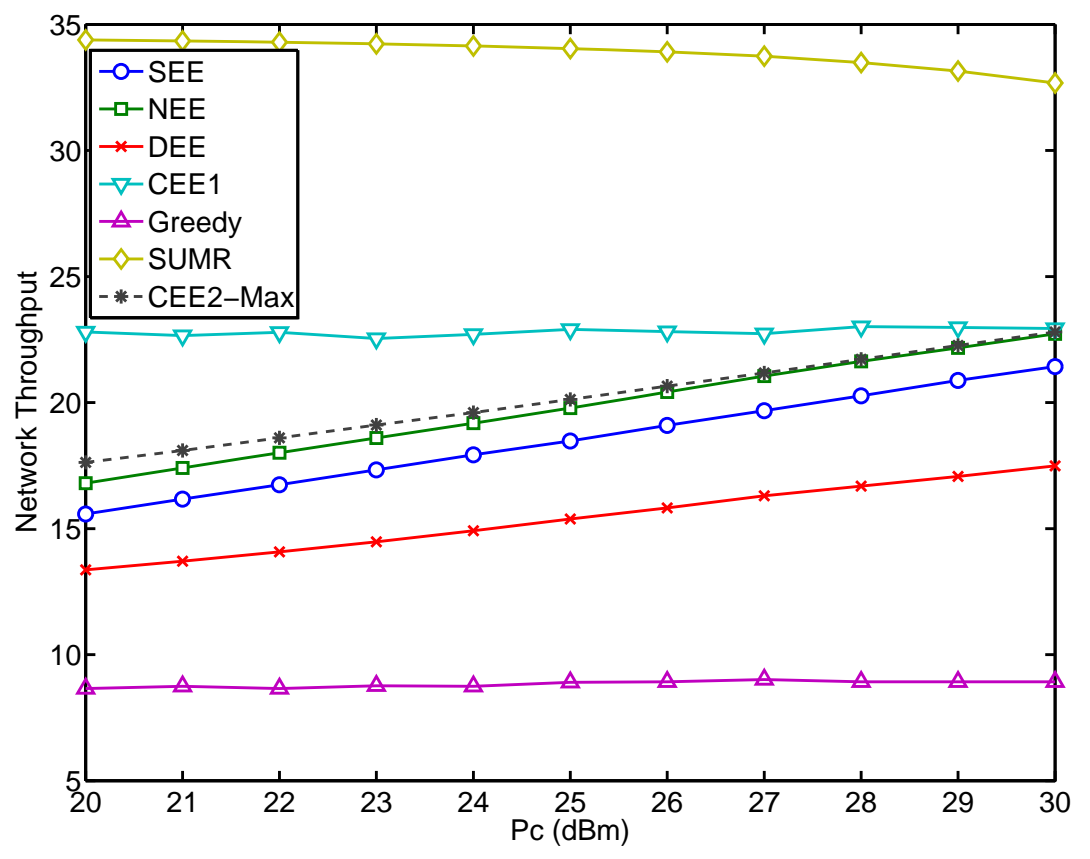

Figure 33: Network throughput in Scenario 2.

power, while the throughput of CEE1 and Greedy almost stays the same. As expected, SUMR has the highest spectrum efficiency. To compare fairness among different links, Figure 34 shows the worst EE among all CR links under different algorithms. It clearly shows that SEE, CEE2-Max and DEE provide better worst-link performance.

Then let both $d_{1}$ and $d_{2}$ be uniformly distributed over $1-50 \mathrm{~m}$ (Scenario 3), which represents a higher interference level than Scenario 2. Figure 35 illustrates the sum of EE of different algorithms. It shows that SEE and CEE2-Max have similar sum of EE performance and outperform the other algorithms, while NEE still achieves higher EE than DEE. Finally, let $d_{2}$ to be uniformly distributed over $1-50 \mathrm{~m}$, while $d_{1}$ to be uniformly distributed over the interval $50-100 \mathrm{~m}$ (Scenario 4). It represents a lower interference level than Scenario 2. The sum of EE of different algorithms is given in Figure 36. It is worth noting that SEE achieves the highest sum of EE among all algorithms. DEE Using 


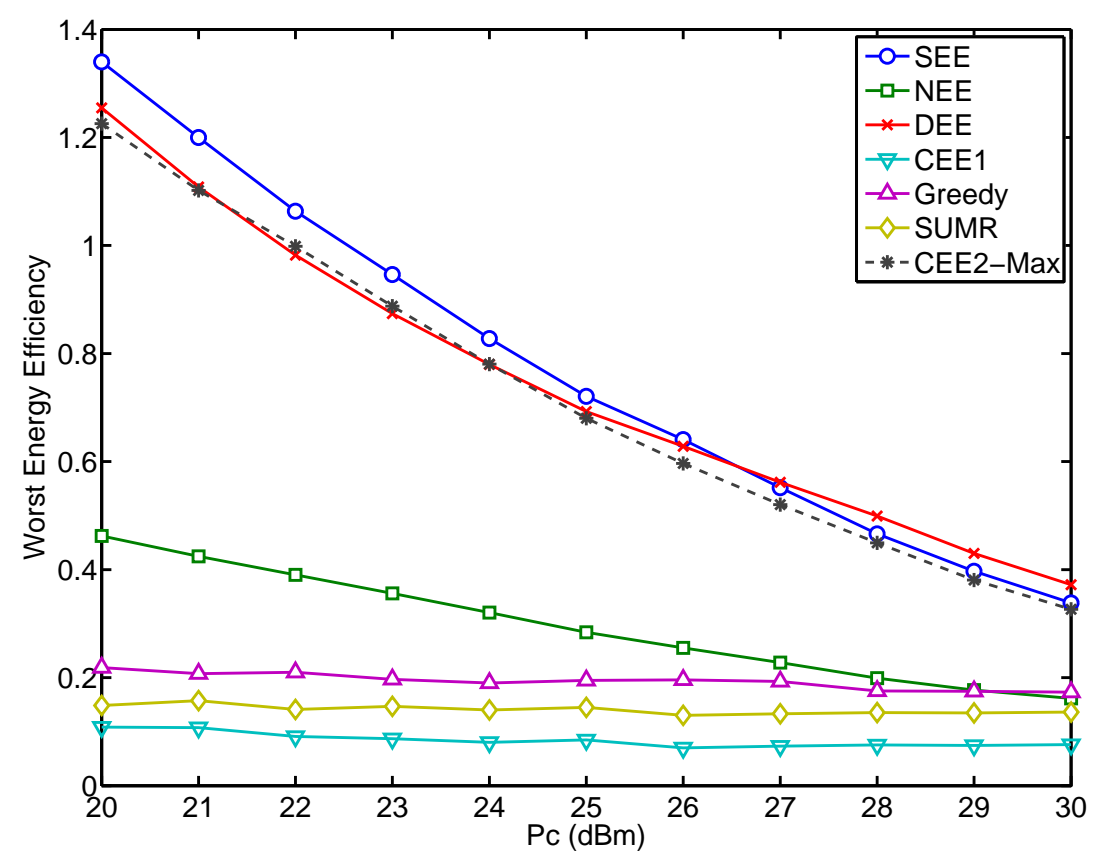

Figure 34: Worst EE under different algorithms in Scenario 2.

only local CSI even outperforms NEE and CEE2-Max, which both require full CSI.

Numerical results show that the proposed algorithms can significantly boost the EE compared to the conventional spectrum efficient transmission strategies. Furthermore, the proposed algorithms also outperform existing energy efficient solutions. Particularly, the sum of EE optimization can achieves equivalent/higher performance in high/low interference channels. The distributed EE algorithm outperforms the existing centralized algorithm under low interference channel. Furthermore, the solution found by proposed algorithms satisfies the maximum power constraint and interference temperature constraint. It means the proposed algorithms are suitable for CR network, in which it demands strict power control to protect the PU's transmission. Meanwhile, the maximum power constraint introduced can assure the resulting transmission strategy within the CR users' power budget. 


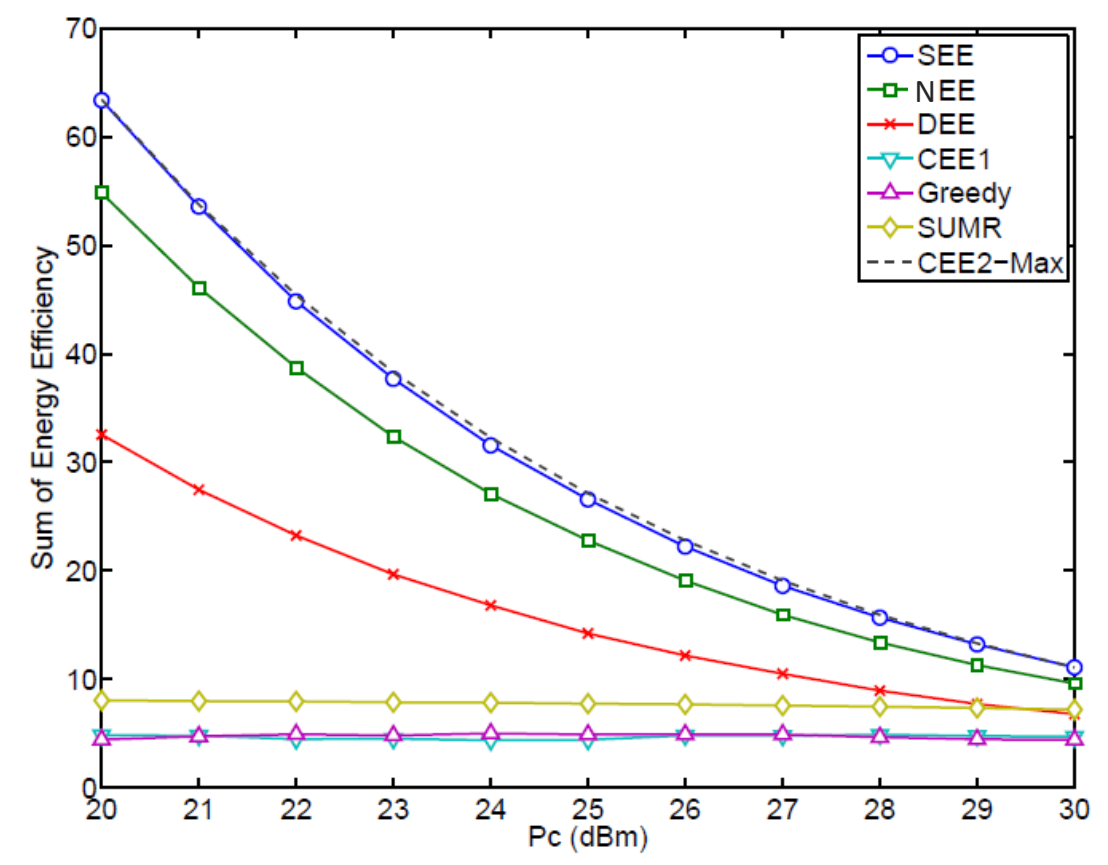

Figure 35: Sum of EE under different algorithms in Scenario 3.

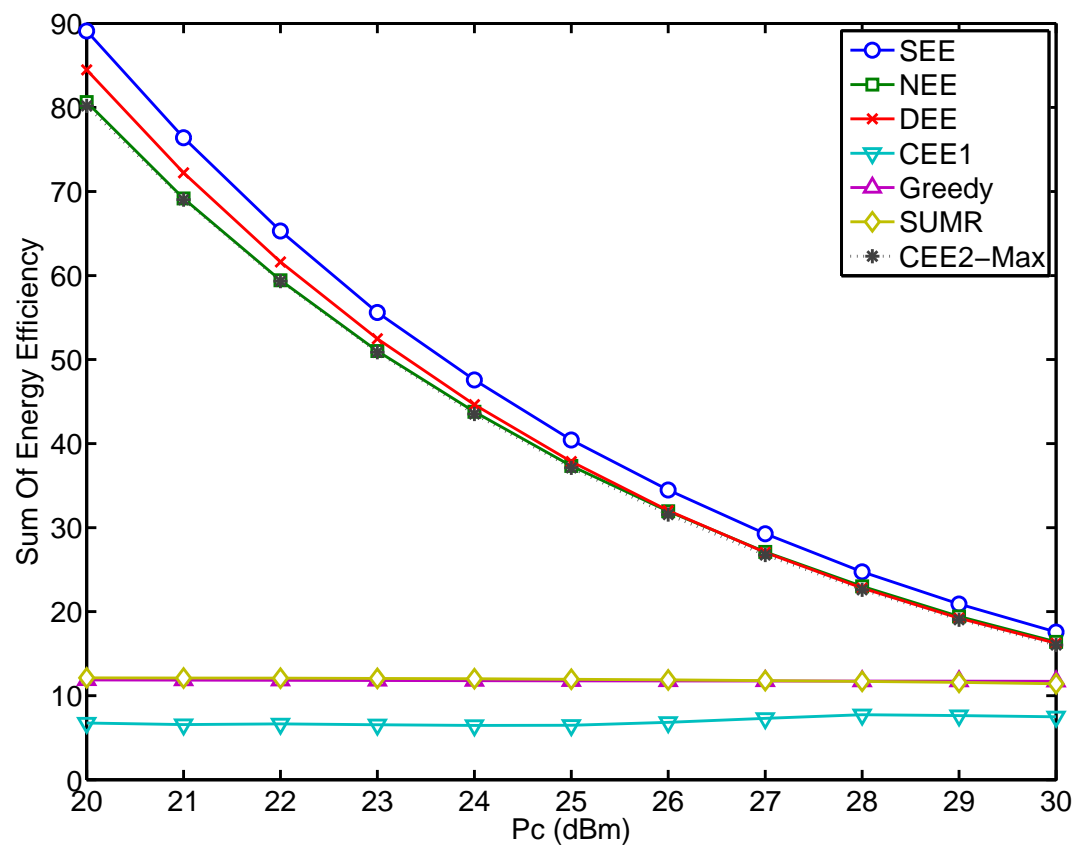

Figure 36: Sum of EE under different algorithms in Scenario 4. 


\section{CHAPTER V}

\section{CONCLUSION AND FUTURE WORK}

In this work, several approaches have been proposed to address the existing challenges for current wireless communication systems. For SC-FDE system, it has introduced frequency domain oversampling into SC-FDE in order to improve the equalization performance of conventional linear receivers under deep fading channel. Also, an iterative IQ imbalance compensation receiver has been designed to combat the IQ imbalance caused by non-ideal transceiver in SC-FDE. For MIMO cognitive radio, energy efficient transmission strategies have been derived to reduce the power consumption of secondary user under interference channels. The conclusion and future works of the corresponding topics are summarized in the Section A and B.

\section{A Single Carrier Frequency Domain Equalization}

For multicarrier system, FDO has been proved as an effective technique to improve the channel equalization performance. However, for single carrier transmission, the impact of FDO was not well studied. In this work, it firstly derives the signal model of SC-FDE system with FDO. It shows that, with proper transceiver design, the effect of frequency selective channel can still be eliminated by an frequency domain one-tap filter, even when the frequency domain signal is oversampled. Two types of FSFD receiver 
based on FDO through LS and MMSE criterion have been proposed. Theoretical and simulation results show that FDO can further extract the frequency diversity and improve the robustness of SC-FDE against deep fading. The BER performance of LS and MMSE based FSFD receivers outperforms the conventional SC-FDE receiver under static channel, Rayleigh fading channel, double-selective channel and imperfect channel knowledge. Furthermore, the performance of frequency domain channel estimation in SC-FDE can also be largely improved by FDO when the pilot sequence is not optimal.

Due to the unideal RF front-end in practice, SC-FDE also suffers from the mirror interference caused by IQ imbalance. Most exiting works on SC-FDE leverages conventional linear receivers to compensate the IQ imbalance. The linear receivers benefit from their low complexity, but their performance is far from optimum. In order to improve the performance of SC-FDE system with IQ imbalance, an iterative decision feedback receiver for SC-FDE has been proposed to compensate the IQ imbalance caused by unideal RF front-end. Both hard detection and soft detection based feedback schemes are considered in the feedforward and feedback filters design. Simulation results have shown that, compared with the conventional MMSE IQ imbalance compensation, significant performance gain can be achieved by introducing decision feedback in the frequency domain for IQ imbalance compensation. The results also have shown that the proposed iterative receiver can achieve similar BER performance with the conventional IBDFE receiver under ideal RF frot-end. Two channel estimation methods have also been derived to validate the effectiveness of the proposed receiver with imperfect channel state information.

The future work of frequency domain oversampling in SC-FDE will be: 
- FSFD receiver under channel impairments: In practice, the communication systems have different impairments that degrade the performance of wireless transmissions. Common channel impairments include frequency/time offset, insufficient guard interval, channel estimation error and IQ imbalance. So it is necessary to investigate the performance of proposed FSFD receiver under different channel impairments. By introducing redundancy in the frequency domain, SC-FDE with FDO may have stronger robustness against the channel impairments than the conventional SC-FDE system.

- Iterative FSFD receiver: In the current work, FSFD receivers are designed according to conventional linear methods (MMSE or LS). Although nonlinear receivers, such as IBDFE, have shown significant advances compared with their linear counterparts, their BER performance still can not meet the optimal match filter bound. From this perspective, the concept of FDO may also be applied to nonlinear receivers for performance improvement. As one of the most promising nonlinear receivers, IBDFE is a good choice to test and verify the effect FDO on nonlinear receivers.

- Iterative IQ imbalance compensation for LTE uplink: The iterative IQ compensation for single carrier transmission can be extended to LTE uplink, which adopts Single Carrier Frequency-Division Multiple Access (SC-FDMA) as a physical layer technique. SC-FDMA can be interpreted as a linearly precoded Orthogonal Frequency-Division Multiple Access (OFDMA) scheme, in the sense that it has an additional DFT processing step preceding the conventional OFDMA processing. It is different with SC-FDE, SC-FDMA deals with the assignment of multiple users to 
a shared communication resource. In addition, the receiver side of SC-FDMA has a

DFT and an IDFT with different lengths. Therefore, the iterative IQ compensation receiver design for SC-FDMA should consider above factors.

\section{B Energy Efficient Transmission Strategy For MIMO Cognitive Radio}

For MIMO cognitive radio network over interference channels, the energy efficiency optimization problems were formulated based on different optimization objectives, which include distributed energy efficiency, network efficiency and sum of individual energy efficiency. Because of the fractional form of the objective functions, these problems are non-convex optimization problems. In order to solve them, they are transformed into relaxed parametric programming problems that are more traceable. Accordingly, three energy-efficient joint power allocation and transmission beamforming algorithms have been developed. The distributed energy efficiency optimization algorithm only needs local CSI while the network energy efficiency and sum of energy efficiency optimization algorithms requires the CR network's full CSI. Numerical results show that the proposed energy-efficient joint power allocation and transmission beamforming strategies can significantly boost the energy efficiency compared to the conventional spectral-efficient transmission strategy. Also, the proposed algorithms can outperform the existing energy efficient solutions. Particularly, the sum of energy efficiency optimization can achieves equivalent/higher performance in high/low interference channels. The distributed energy efficiency algorithm, which only needs local CSI, can outperform the existing centralized algorithm under low interference channels. The performance gap between distributed algorithms and the centralized algorithms for energy efficiency 
optimization decreases as the interference level goes down. In addition, the solutions found by proposed algorithms satisfy the maximum power constraint and interference temperature constraint, consequently, can protect the PU's transmission.

The future work of energy efficiency optimization will be:

- Energy efficiency optimization with limited CSI feedback: The current work on the energy efficiency optimization requires the full CSI knowledge at transmitters. Since channel estimation is usually performed at the receiver side, the full CSI knowledge requirement at the transmitter side will cause significant CSI feedback overhead in practice. To address this issue, energy efficiency optimization with limited CSI feedback should be considered in the future work.

- Sum of energy efficiency optimization in multiuser OFDMA network: The parametric method for solving the sum of energy efficiency optimization problem can be extended to other network models, such as multiuser OFDMA network. OFDMA is a multiple-access technique capable of allowing multiple users to simultaneously transmit on different subcarriers. Therefore, the energy efficiency optimization of multiuser OFDMA can also be formulated as a sum of ratio form. 


\section{REFERENCES}

[1] L. Cimini, "Analysis and simulation of a digital mobile channel using orthogonal frequency division multiplexing," IEEE Transactions on Communications, vol. 33, pp. 665-675, Jul 1985.

[2] D. Falconer, S. Ariyavisitakul, A. Benyamin-Seeyar, and B. Eidson, "Frequency domain equalization for single-carrier broadband wireless systems," IEEE Communications Magazine, vol. 40, pp. 58-66, Apr 2002.

[3] H. Sari, G. Karam, and I. Jeanclaude, "Transmission techniques for digital terrestrial tv broadcasting," IEEE Communications Magazine, vol. 33, pp. 100-109, Feb 1995.

[4] A. Czylwik, "Comparison between adaptive ofdm and single carrier modulation with frequency domain equalization," in IEEE 47th Vehicular Technology Conference, 1997, vol. 2, pp. 865-869 vol.2, May 1997.

[5] V. Lottici, M. Luise, M. Marselli, and R. Reggiannini, "Blind subcarrier frequency ambiguity resolution for ofdm signals over selective channels," IEEE Transactions on Communications, vol. 52, pp. 1532-1537, Sept 2004.

[6] "Multiple-access interference suppression for mc-cdma by frequency-domain oversampling," IEEE Transactions on Communications, vol. 53, pp. 545-545, March 2005.

[7] Q. Shi, Y. L. Guan, Y. Gong, and C. Law, "Receiver design for multicarrier cdma using frequency-domain oversampling," IEEE Transactions on Wireless Communications, vol. 8, pp. 2236-2241, May 2009.

[8] Q. Shi, L. Liu, Y. L. Guan, and Y. Gong, "Fractionally spaced frequency-domain mmse receiver for ofdm systems," IEEE Transactions on Vehicular Technology, vol. 59, pp. 4400-4407, Nov 2010.

[9] Z. Wang, S. Zhou, G. Giannakis, C. Berger, and J. Huang, "Frequency-domain oversampling for zero-padded ofdm in underwater acoustic communications," IEEE Journal of Oceanic Engineering, vol. 37, pp. 14-24, Jan 2012.

[10] J. Zheng and Y. Sun, "Two-way amplify forward relaying using frequencydomain oversampling on frequency-selective fading channels," IEEE Transactions on Vehicular Technology, vol. 63, pp. 1982-1987, May 2014.

[11] J. Andrews, S. Buzzi, W. Choi, S. Hanly, A. Lozano, A. Soong, and J. Zhang, "What will $5 \mathrm{~g}$ be?," IEEE Journal on Selected Areas in Communications, vol. 32, pp. 10651082, June 2014. 
[12] O. Onireti, F. Heliot, and M. Imran, "On the energy efficiency-spectral efficiency trade-off of distributed mimo systems," IEEE Transactions on Communications, vol. 61, pp. 3741-3753, September 2013.

[13] C. Stevenson, G. Chouinard, Z. Lei, W. Hu, S. Shellhammer, and W. Caldwell, "Ieee 802.22: The first cognitive radio wireless regional area network standard," IEEE Communications Magazine, vol. 47, pp. 130-138, January 2009.

[14] L. Zheng and D. Tse, "Diversity and multiplexing: a fundamental tradeoff in multipleantenna channels," IEEE Transactions on Information Theory, vol. 49, pp. 10731096, May 2003.

[15] Y. Zheng and C. Xiao, "Channel estimation for frequency-domain equalization of single-carrier broadband wireless communications," IEEE Transactions on Vehicular Technology, vol. 58, pp. 815-823, Feb 2009.

[16] A. Czylwik, "Comparison between adaptive ofdm and single carrier modulation with frequency domain equalization," in IEEE 47th Vehicular Technology Conference, vol. 2, pp. 865-869 vol.2, May 1997.

[17] A. Mehana and A. Nosratinia, "Single-carrier frequency-domain equalizer with multiantenna transmit diversity," IEEE Transactions on Wireless Communications, vol. 12, pp. 388-397, January 2013.

[18] J. G. Proakis, Digital Communications. New York: McGraw-Hill, 1995.

[19] X. Zhang, E. Chen, and X. Mu, "Single-carrier frequency-domain equalization based on frequency-domain oversampling," IEEE Communications Letters, vol. 16, pp. 24 26, January 2012.

[20] A. Gusmao, R. Dinis, and N. Esteves, "On frequency-domain equalization and diversity combining for broadband wireless communications," IEEE Transactions on Communications, vol. 51, pp. 1029-1033, July 2003.

[21] A. Tajer and A. Nosratinia, "Diversity order in isi channels with single-carrier frequency-domain equalizers," IEEE Transactions on Wireless Communications, vol. 9, pp. 1022-1032, March 2010.

[22] A. V. Oppenheim, Discrete-Time Signal Processing. Pearson Education, 2006.

[23] S. Kim, K. Lee, J. Cha, J. Kang, N. Kim, and S. Park, "Frequency domain equalizer using zero-padding for ir-uwb systems," in IEEE International Conference on UltraWideband, pp. 905-909, Sept 2007.

[24] I. Prokhorov and V. Statulevicius, Limit Theorems of Probability Theory. Encyclopaedia of Mathematical Sciences, Springer, 2000.

[25] M. Simon and M. Alouini, Digital Communication over Fading Channels. Wiley Series in Telecommunications and Signal Processing, Wiley, 2005. 
[26] A. Chini and C. University, Multi Carrier Modulation in Frequency Selective Fading Channels. UMI, 1994.

[27] H. Sari, G. Karam, and I. Jeanclaude, "Transmission techniques for digital terrestrial tv broadcasting," IEEE Communications Magazine, vol. 33, pp. 100-109, Feb 1995.

[28] X. Fernando and A. Sesay, "A hammerstein-type equalizer for concatenated fiberwireless uplink," IEEE Transactions on Vehicular Technology, vol. 54, pp. 19801991, Nov 2005.

[29] J. Coon, M. Beach, J. McGeehan, and M. Sandell, "Channel and noise variance estimation and tracking algorithms for unique-word based single-carrier systems," in 1st International Symposium on Wireless Communication Systems, pp. 51-55, Sept 2004.

[30] X. Zhang, E. Chen, and X. Mu, "Single-carrier frequency-domain equalization based on frequency-domain oversampling," IEEE Commun. Lett., vol. 16, pp. 24-26, January 2012.

[31] "Ieee p802.11- task group ad."

[32] "Ieee 802.15.3c-2009: Part 15.3: Wireless medium access control (mac) and physical layer (phy) specifications for high rate wireless personal area networks (wpans)."

[33] V. Rampa, "I/Q compensation of broadband direct-conversion transmitters," IEEE Trans. Wireless Commun., vol. 13, pp. 3329-3342, Jun 2014.

[34] B. Narasimhan, D. Wang, S. Narayanan, H. Minn, and N. Al-Dhahir, "Digital compensation of frequency-dependent joint Tx/Rx I/Q imbalance in OFDM systems under high mobility," IEEE J. Sel. Topics Signal Process., vol. 3, pp. 405-417, Jun 2009.

[35] C.-L. Liu, "Impacts of I/Q imbalance on QPSK-OFDM-QAM detection," IEEE Trans. Consum. Electron., vol. 44, pp. 984-989, Aug 1998.

[36] M. Windisch and G. Fettweis, "Performance degradation due to I/Q imbalance in multi-carrier direct conversion receivers: a theoretical analysis," in Proc. IEEE International Conference on Communications, vol. 1, pp. 257-262, Jun 2006.

[37] F. Lopez-Martinez, E. Martos-Naya, J. Paris, and J. Entrambasaguas, "Exact closedform BER analysis of OFDM systems in the presence of IQ imbalances and ICSI," IEEE Trans. Wireless Commun., vol. 10, pp. 1914-1922, Jun 2011.

[38] M. Valkama, M. Renfors, and V. Koivunen, "Advanced methods for I/Q imbalance compensation in communication receivers," IEEE Trans. Signal Process., vol. 49, pp. 2335-2344, Oct 2001.

[39] A. Tarighat, R. Bagheri, and A. Sayed, "Compensation schemes and performance analysis of IQ imbalances in OFDM receivers," IEEE Trans. Signal Process., vol. 53, 
pp. 3257-3268, Aug 2005.

[40] D. Mattera, L. Paura, and F. Sterle, "MMSE WL equalizer in presence of receiver IQ imbalance," IEEE Trans. Signal Process., vol. 56, pp. 1735-1740, Apr 2008.

[41] S. Traverso, M. Ariaudo, I. Fijalkow, J.-L. Gautier, and C. Lereau, "Decision-directed channel estimation and high I/Q imbalance compensation in OFDM receivers," IEEE Trans. Commun., vol. 57, pp. 1246-1249, May 2009.

[42] M. Marey, M. Samir, and O. Dobre, "EM-based joint channel estimation and IQ imbalances for OFDM systems," IEEE Trans. Broadcast., vol. 58, pp. 106-113, Mar 2012.

[43] M. Marey, M. Samir, and M. Ahmed, "Joint estimation of transmitter and receiver IQ imbalance with ML detection for Alamouti OFDM systems," IEEE Trans. Veh. Technol., vol. 62, pp. 2847-2853, Jul 2013.

[44] S. Narayanan, B. Narasimhan, and N. Al-Dhahir, "Training sequence design for joint channel and I/Q imbalance parameter estimation in mobile SC-FDE transceivers," in Proc. IEEE International Conference on Acoustics Speech and Signal Processing (ICASSP), pp. 3186-3189, Mar 2010.

[45] W. Hou and M. Jiang, "Enhanced joint channel and IQ imbalance parameter estimation for mobile communications," IEEE Commun. Lett., vol. 17, pp. 13921395, Jul 2013.

[46] F. Horlin and A. Bourdoux, "Comparison of the sensitivity of OFDM and SCFDE to CFO, SCO and IQ imbalance," in Proc. International Symposium on Communications, Control and Signal Processing (ISCCSP), pp. 111-116, Mar 2008.

[47] S. Narayanan, B. Narasimhan, and N. Al-Dhahir, "Baseband estimation and compensation of joint TX/RX I/Q imbalance in SC-FDE transceivers," in Information Sciences and Systems, 2009. CISS 2009. 43rd Annual Conference on, pp. 551-556, March 2009.

[48] C. Zhang, Z. Xiao, B. Gao, L. Su, and D. Jin, "Three-stage treatment of TX/RX IQ imbalance and channel with CFO for SC-FDE systems," IEEE Commun. Lett., vol. 18, pp. 297-300, Feb 2014.

[49] X. Cheng, Z. Luo, and S. Li, "Joint estimation for I/Q imbalance and multipath channel in millimeter-wave SC-FDE systems," IEEE Trans. Veh. Technol., vol. PP, no. 99, pp. 1-1, 2015.

[50] N. Souto, R. Dinis, A. Correia, and C. Reis, "Interference-aware iterative block decision feedback equalizer for single-carrier transmission," IEEE Trans. Veh. Technol., vol. 64, pp. 3316-3321, Jul 2015.

[51] N. Benvenuto and S. Tomasin, "Iterative design and detection of a DFE in the 
frequency domain," IEEE Trans. Commun., vol. 53, pp. 1867-1875, Nov 2005.

[52] N. Souto, R. Dinis, and J. Silva, "Impact of channel estimation errors on SC-FDE systems," IEEE Trans. Commun., vol. 62, pp. 1530-1540, May 2014.

[53] A. Gusmao, P. Torres, R. Dinis, and N. Esteves, "A turbo FDE technique for reducedCP SC-based block transmission systems," IEEE Trans. Commun., vol. 55, pp. 16-20, Jan 2007.

[54] R. Dinis, P. Montezuma, N. Souto, and J. Silva, "Iterative frequency-domain equalization for general constellations," in Proc. IEEE Sarnoff Symposium, pp. 1-5, Apr 2010.

[55] G. Gong, F. Huo, and Y. Yang, "Large zero autocorrelation zones of Golay sequences and their applications," IEEE Trans. Commun., vol. 61, pp. 3967-3979, Sep 2013.

[56] L. Anttila, M. Valkama, and M. Renfors, "Frequency-selective I/Q mismatch calibration of wideband direct-conversion transmitters," IEEE Trans. Circuits Syst. II, vol. 55, pp. 359-363, April 2008.

[57] J. W. Kang, Y. Whang, B. H. Ko, and K. S. Kim, "Generalized cross-correlation properties of Chu sequences," IEEE Trans. Inf. Theory, vol. 58, pp. 438-444, Jan 2012.

[58] S. Haykin, "Cognitive radio: brain-empowered wireless communications," IEEE Journal on Selected Areas in Communications, vol. 23, pp. 201-220, Feb 2005.

[59] Y.-C. Liang, K.-C. Chen, G. Li, and P. Mahonen, "Cognitive radio networking and communications: an overview," IEEE Transactions on Vehicular Technology, vol. 60, pp. 3386-3407, Sept 2011.

[60] G. Scutari, D. Palomar, and S. Barbarossa, "Cognitive mimo radio," IEEE Signal Processing Magazine, vol. 25, pp. 46-59, November 2008.

[61] D. Nguyen and M. Krunz, "Power minimization in mimo cognitive networks using beamforming games," IEEE Journal on Selected Areas in Communications, vol. 31, pp. 916-925, May 2013.

[62] D. Nguyen and M. Krunz, "Price-based joint beamforming and spectrum management in multi-antenna cognitive radio networks," IEEE Journal on Selected Areas in Communications, vol. 30, pp. 2295-2305, December 2012.

[63] G. Scutari and D. Palomar, "Mimo cognitive radio: A game theoretical approach," IEEE Transactions on Signal Processing, vol. 58, pp. 761-780, Feb 2010.

[64] Y. Liu and L. Dong, "Spectrum sharing in mimo cognitive radio networks based on cooperative game theory," IEEE Transactions on Wireless Communications, vol. 13, pp. 4807-4820, Sept 2014. 
[65] J. Mao, J. Gao, Y. Liu, G. Xie, and X. Li, "Power allocation over fading cognitive mimo channels: An ergodic capacity perspective," IEEE Transactions on Vehicular Technology, vol. 61, pp. 1162-1173, March 2012.

[66] Y. Noam, A. Manolakos, and A. Goldsmith, "Null space learning with interference feedback for spatial division multiple access," IEEE Transactions on Wireless Communications, vol. 13, pp. 5699-5715, Oct 2014.

[67] X. Huang, B. Beferull-Lozano, and C. Botella, "Non-convex power allocation games in mimo cognitive radio networks," in 2013 IEEE 14th Workshop on Signal Processing Advances in Wireless Communications (SPAWC), pp. 145-149, June 2013.

[68] B. Kollimarla and Q. Cheng, "Adaptive pricing for efficient spectrum sharing in mimo systems," in 2010 IEEE 71st Vehicular Technology Conference (VTC 2010-Spring), pp. 1-5, May 2010.

[69] L. Fu, Y. Zhang, and J. Huang, "Energy efficient transmissions in mimo cognitive radio networks," IEEE Journal on Selected Areas in Communications, vol. 31, pp. 2420-2431, November 2013.

[70] W. Zhong and J. Wang, "Energy efficient spectrum sharing strategy selection for cognitive mimo interference channels," IEEE Transactions on Signal Processing, vol. 61, pp. 3705-3717, July 2013.

[71] C. Jiang and L. Cimini, "Energy-efficient transmission for mimo interference channels," IEEE Trans. Wireless Commun., vol. 12, pp. 2988-2999, June 2013.

[72] S. Wang, M. Ge, and W. Zhao, "Energy-efficient resource allocation for ofdm-based cognitive radio networks," IEEE Transactions on Communications, vol. 61, pp. 31813191, August 2013.

[73] K. Cheung, S. Yang, and L. Hanzo, "Achieving maximum energy-efficiency in multi-relay ofdma cellular networks: A fractional programming approach," IEEE Transactions on Communications, vol. 61, pp. 2746-2757, July 2013.

[74] H. P. Benson, "Global optimization of nonlinear sums of ratios," Journal of Mathematical Analysis and Applications, vol. 263, no. 1, pp. 301 - 315, 2001.

[75] C. Xiong, L. Lu, and G. Li, "Energy-efficient spectrum access in cognitive radios," IEEE Journal on Selected Areas in Communications, vol. 32, pp. 550-562, March 2014.

[76] X. Ge, X. Huang, Y. Wang, M. Chen, Q. Li, T. Han, and C.-X. Wang, "Energyefficiency optimization for mimo-ofdm mobile multimedia communication systems with qos constraints," IEEE Transactions on Vehicular Technology, vol. 63, pp. 21272138, Jun 2014.

[77] W. Dinkelbach, "On nonlinear fractional programming," Management Science, 
vol. 13, no. 7, pp. 492-498, 1967.

[78] R. Freund and F. Jarre, "Solving the sum-of-ratios problem by an interior-point method," Journal of Global Optimization, vol. 19, no. 1, pp. 83-102, 2001.

[79] Y. Jong, "Practical global optimization algorithm for the sum-of-ratios problem," arXiv, 2012.

[80] S. He, Y. Huang, S. Jin, and L. Yang, "Coordinated energy-efficient precoding for cr mimo interference channels," in 2014 IEEE Global Conference on Signal and Information Processing (GlobalSIP), pp. 183-187, Dec 2014.

[81] J. Wang, G. Scutari, and D. Palomar, "Robust mimo cognitive radio via game theory," IEEE Transactions on Signal Processing, vol. 59, pp. 1183-1201, March 2011. 


\section{CURRICULUM VITAE}

NAME: $\quad$ Xiaohui Zhang

ADDRESS: $\quad$ W.S. Speed Hall

University of Louisville

Louisville, KY 40208

EDUCATION: $\quad$ Ph.D. Electrical Engineering, University of Louisville, 2016

B.S. Electrical Engineering, Zhengzhou University, 2008

M.S. Electrical Engineering, Zhengzhou University, 2012

RESEARCH: Wireless Communication, Signal Processing

Optimization, Single Carrier Transmissions

Cognitive Radio, MIMO, Energy Efficiency

TEACHING: $\quad$ ECE550 Communication and Modulation - LTA/GTA

ECE 252 Introduction to Electrical Engineering - GTA

\section{PUBLICATION:}

[1] Xiahui Zhang, Hongxiang Li, “ Centralized Energy Efficient Transmission Strategy MIMO-CR Network Under Interference Channels”, Preparing for submission on IEEE Transaction on Wireless Communication.

[2] Xiahui Zhang, Hongxiang Li, "Iterative IQ imbalance Compensation Receiver for Single Carrier Transmission," Accepted under Minor Revision by IEEE Transactions on Vehicular Technology, 2016. 
[3] Chen Cao, Hongxiang Li, Zixia Hu, Wenqi Liu and Xiaohui Zhang, "Physical-Layer Secrecy Performance in Finite Blocklength Case," 2015 IEEE Global Communications Conference (GLOBECOM), pp. 1-6, San Diego, CA, 2015.

[4] Xiaohui Zhang, Hongxiang Li, Yanhui Lu, Bing Zhou, "Distributed Energy Efficiency Optimization for MIMO Cognitive Radio Network," IEEE Communications Letters, vol.19, no.5, pp.847-850, May 2015.

[5] Xioahui Zhang, Hongxiang Li, "Energy Efficiency Optimization for MIMO Cognitive Radio Network," 2015 IEEE International Conference on Communications (ICC), pp. 7713-7718, London, 2015.

[6] Xiaohui Zhang, Enqing Chen, Xiaomin Mu, "Single-Carrier Frequency-Domain Equalization Based on Frequency-Domain Oversampling," IEEE Communications Letters, vol.16, no.1, pp.24-26, January 2012. 